\title{
REAL AND COMPLEX DYNAMICS OF A FAMILY OF BIRATIONAL MAPS OF THE PLANE: THE GOLDEN MEAN SUBSHIFT
}

\author{
ERIC BEDFORD \& JEFFREY DILLER
}

\begin{abstract}
Contents

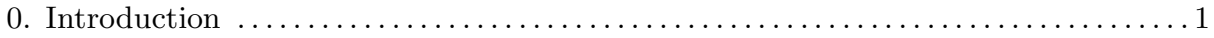

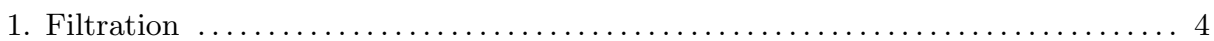

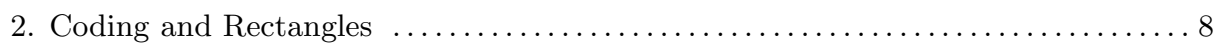

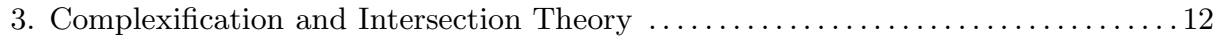

4. Structure of Rectangles .......................................... 14

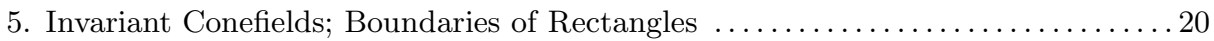

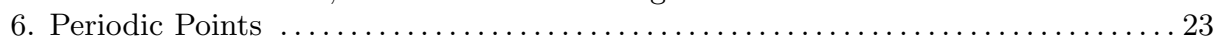

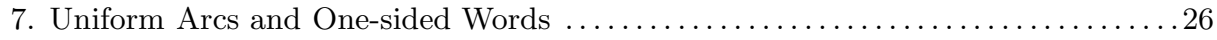

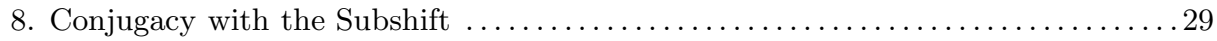

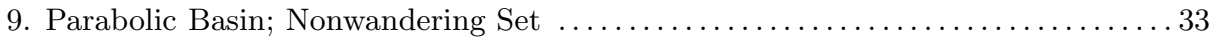

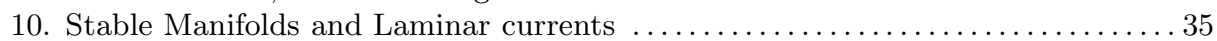

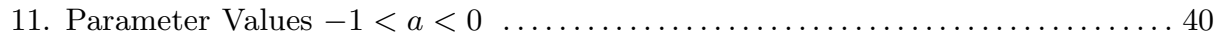

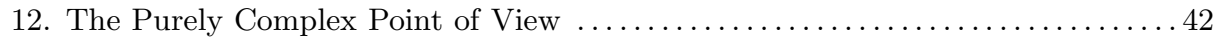

\section{INTRODUCTION}

Much attention has been given to birational mappings which arise in connection with integrable systems; two recent surveys of this subject are given in [BTR] and [GNR]. For these birational mappings, it is of interest to know the behavior of the iterates $f^{n}=f \circ \cdots \circ f$ as $n$ increases. A family which comes from the study of lattice statistical mechanics (see $[\mathrm{BM}]$ ) is

$$
f_{a}(x, y)=\left(y \frac{x+a}{x-1}, x+a-1\right)
$$

for fixed $a \in \mathbf{R}$, which defines a birational mapping of the plane $\mathbf{R}^{2}$. (Throughout this paper we exclude the case $a=-1$ since $f_{-1}$ is affine and thus dynamically trivial.) Typical of mappings that arise this way, $f_{a}$ is area-preserving in the sense that it preserves a meromorphic 2-form, and is reversible, which means that $f_{a}$ is conjugate to $f_{a}^{-1}$ via an involution. This family was investigated in a series of papers by Abarenkova et al. [Ab1-5], which describe several numerical phenomena and raise a number of interesting questions.

The goal of this paper is to give a precise description of the dynamics of $f_{a}$ for $a<0$. One of our motivations here is to give a first example of pointwise dynamics of a birational mapping which is, in an essential way, not a homeomorphism of its nonwandering set. One of the properties of a 
rational map is that it can have points of indeterminacy: at such points the mapping cannot be defined to take a single value, and the map is said to "blow up" such points, assigning whole curves to them.

One way of dealing with such behavior is to consider the closed relation induced by $f_{a}$ and to work within the general framework of topological dynamics (see Akin $[\mathrm{Ak}]$ ). This framework, however, does not reflect the rich structure of a birational map, so we also work with the complexification $\tilde{f}_{a}$, which is a birational map of $\mathbf{C}^{2}$. This allows us to use the tools of complex analysis, complex geometry, and complex potential theory. Despite pointwise difficulties, $f_{a}$ induces a well-defined map $f_{a}^{*}$ on the set of positive closed (1,1)-currents (see Sibony [S] and Guedj [Gu1]). In [DF] it was shown that for all values $a \in \mathbf{C}-\left\{-1,0, \frac{1}{3}, \frac{1}{2}, 1\right\}$ there is an invariant (1,1)-current $T_{a}^{+}$such that $\tilde{f}_{a}^{*} T_{a}^{+}=\rho_{a} T_{a}^{+}$, with $\rho_{a}>1$. It follows that the degrees of $f_{a}^{n}$ grow exponentially like $\rho_{a}^{n}$, thus confirming a conjecture of [Ab1-5]. Except for a countable set of exceptional values of $a, \rho_{a}$ is equal to the golden mean $\phi:=(1+\sqrt{5}) / 2$.

Measures (which correspond to 0-currents) transform differently from $(1,1)$-currents and thus reflect more closely the pointwise behavior of the map. A construction of invariant measures for a rather general class of birational mappings, which includes the family $\left\{\tilde{f}_{a}\right\}$ is given in [BD]. In this paper we are able to go farther and give a pointwise description of the dynamics. In particular, we describe the behavior of $f_{a}$ on the indeterminacy and critical sets, both of which are "invisible" from the measure-theoretic point of view.

Since the coordinate functions of $f=f_{a}$ are rational, we extend $f_{a}$ to the compactification $\overline{\mathbf{R}^{2}}:=(\mathbf{R} \cup\{\infty\}) \times(\mathbf{R} \cup\{\infty\})$. The point $(\infty, \infty)$ is a parabolic fixed point for $f$. The forward/backward basin $\mathcal{B}_{ \pm}$is the set of points where $f^{n}$ converges locally uniformly to $(\infty, \infty)$ as $n \rightarrow \pm \infty$. We prove in Section 9 that the nonwandering set is the complement of the parabolic basin $\mathcal{B}_{+} \cup \mathcal{B}_{-}$.

Our abstract model for the dynamics of $f$ on the nonwandering set will be the so-called golden mean subshift $(\sigma, \Sigma)$. That is, $\sigma$ is the shift map, and $\Sigma$ is the topological space of bi-infinite sequences of 0 's and 1's such that ' 1 ' is always followed by ' 0 '. The entropy of this subshift is the logarithm of the golden mean $\phi$. We connect $f_{a}$ with our model system by giving a (multiplevalued) equivariant correspondence $R$ taking $(\sigma, \Sigma)$ to $(f, \Omega)$. Note that $R$ cannot be a topological conjugacy because $(\sigma, \Sigma)$ is a homeomorphism while $(f, \Omega)$ is not.

We identify rectangles $R_{0}$ and $R_{1}$ which cover $\Omega$ and which serve as a Markov partition. Any $f$-orbit $\left(p_{n}\right)_{n \in \mathbf{Z}}$ which lies in $\Omega-(\infty, \infty)$ can be assigned a coding $w=\left(w_{n}\right)_{n \in \mathbf{Z}}$, where each symbol $w_{n} \in\{0,1\}$ is chosen so that $p_{n} \in R_{w_{n}}$. By the mapping properties of $R_{0}$ and $R_{1}$, it follows that '11' cannot occur, and thus $w \in \Sigma$. We adopt the convention of coding the fixed point $(\infty, \infty) \in \Omega$ by the 2 -cycle $\overline{01} \leftrightarrow \overline{10}$ in $\Sigma$. As it turns out, we will find it more convenient to work with the set-theoretic "inverse" of the coding map. Let $R(w)$ be the "rectangle" of points coded by $w$. More precisely, we set

$$
R(w)=\bigcap_{n \in \mathbf{Z}} f^{-n} R_{w_{n}}-(\infty, \infty)
$$


for $w \in \Sigma-\{\overline{01}, \overline{10}\}$, and we set $R(\overline{01})=R(\overline{10})=(\infty, \infty) . \quad R$ defines a semi-conjugacy from $(\sigma, \Sigma)$ to $(f, \Omega)$ in the following sense: If $R(w)$ does not contain the point of indeterminacy $(-a, \infty)$, then

$$
f R(w)=R(\sigma w) .
$$

There is a unique word $w_{*}$ such that $R\left(w_{*}\right)=E$ is a nontrivial interval containing a point of indeterminacy (see Figure 6). One of our principal results is Theorem 8.1 which says that if $w \neq \sigma^{n} w_{*}$, then $R(w)$ is a single point.

Now we may further describe the dynamics of $f: f^{2}$ acts by translation on the two lines at infinity, so the behavior is decidedly non-hyperbolic everywhere on $\overline{\mathbf{R}^{2}}-\mathbf{R}^{2}$. On the other hand, the behavior of $f$ on $\Omega \cap \mathbf{R}^{2}$ has many of the properties of an Axiom A diffeomorphism:

(1) There are invariant cone fields for $f$ at all points of $\Omega \cap \mathbf{R}^{2}$.

(2) All periodic points, except for $(\infty, \infty)$, belong to $\mathbf{R}^{2}$ and are saddle points.

(3) The saddle points are a dense subset of $\Omega \cap \mathbf{R}^{2}$.

(4) $f$ is topologically expansive on $\Omega \cap \mathbf{R}^{2}$.

(5) There are stable and unstable manifolds through every point of $\Omega \cap$ $\mathbf{R}^{2}$; the corresponding laminations, $\mathcal{W}^{s}$ and $\mathcal{W}^{u}$, intersect transversally.

Let us reiterate that the cone field and expansivity mentioned above are defined only on $\Omega \cap \mathbf{R}^{2}$, which is not an invariant set. Note, too, that distinct stable manifolds intersect at points of the countable set $I_{+}=\bigcup_{n \geq 0} f^{-n} I(f)$; see Figures 9 and 11 .

Finally, we draw a parallel with a result of Ruelle and Sullivan [RS] for Axiom A surface mappings; we show that $\mathcal{W}^{s}$ and $\mathcal{W}^{u}$ can be used to construct a "stable current" $\mu_{\mathbf{R}}^{+}$and an "unstable current" $\mu_{\mathbf{R}}^{-}$whose intersection product gives the unique measure of maximal entropy. These currents should give a connection between the real dynamics of $f_{a}$ and the complex dynamics of $\tilde{f}_{a}$ because the invariant current $T_{a}^{+}$(which has real dimension 2) appears to be the "complexification" of the 1-dimensional current $\mu_{\mathbf{R}}^{+}$.

Let us outline our mathematical approach. We work simultaneously with the real map $f_{a}$ and its complexification $\tilde{f}_{a}$. We consider the forward/backward iterates of complex lines in $\mathbf{C}^{2}$. Let $\tilde{L}$ and $\tilde{M}$ denote the complexifications of real lines $L$ and $M$. By the intersection theory of complex subvarieties, we know that the intersection number of $\tilde{f}^{-n} \tilde{L}$ and $\tilde{f}^{m} \tilde{M}$ is determined by the homology classes of these two sets. Considering the purely real behavior, we develop a geometric/combinatorial argument which gives a lower bound on the number of (real) intersection points of $f^{-n} L \cap f^{m} M$. This allows us to conclude that $R(w)$ is nonempty. This lower bound coincides with the upper bound given by complex intersection theory; hence all intersection points are real and have multiplicity one. The property of having multiplicity one leads to transversality and the existence of cone fields.

We believe that the maps $\left\{f_{a}: a<0\right\}$ represent an important subfamily within the whole family $\left\{f_{a}\right\}$. This may be seen by analogy with the Hénon family $\left\{h_{a, b}: b \neq 0\right\}$

$$
h_{a, b}(x, y)=\left(a-x^{2}-b y, x\right)
$$


of quadratic diffeomorphisms of $\mathbf{R}^{2}$. For $a \ll-1$, the map $h_{a, b}$ has dynamics which are completely transient: all orbits tend to $\infty$. On the other hand, it was shown in $[\mathrm{DN}]$ that $h_{a, b}$ generates a horseshoe when $a \gg 1$. [HO], using complex methods, were able to obtain a much larger family of horseshoes. By Friedland and Milnor [FM] it is known that the entropy of $h_{a, b}$ is no greater than $\log 2$, and thus the horseshoe mappings in $\left\{h_{a, b}\right\}$ represent elements of maximal entropy. The transition of behaviors of $h_{a, b}$ as $a$ passes from $a \ll-1$ to $a \gg 1$ is seen as illustrating a mechanism for the transition to chaos, with the horseshoe mappings exhibiting fully developed chaos. The central position of the horseshoe in this picture is given from the point of view of the "Pruning Front Conjecture" by de Carvalho and Hall in $[\mathrm{dCH}]$. The point of our analogy here is that the maps $\left\{f_{a}, a<0\right\}$ have maximal entropy within the family $\left\{f_{a}: a \neq-1\right\}$, and we expect them to play a fundamental role within this family, as the horseshoes play within the Hénon family. On the technical level, too, we have borrowed from the analogy with the Hénon family. It was shown in [BLS] that if $h_{a, b}$ is of maximal entropy, then the nonwandering set for the complexification is contained in $\mathbf{R}^{2}$. In [BS1,2], it was shown that a mapping $h_{a, b}$ with maximal entropy may be studied by working with its complexification; that approach has motivated some of the work of the current paper.

\section{Filtration}

Throughout this paper we consider $f_{a}$ only for $a<0, a \neq-1$. In fact, we will assume that $a<-1$ until we reach Section 11], where we indicate the modifications needed to treat the case $-1<a<0$. We write $f=f_{a}$; the inverse of $f$ is given by

$$
f^{-1}(x, y)=\left(y+1-a, x \frac{y-a}{y+1}\right) .
$$

The involution $(x, y) \mapsto(-y,-x)$ conjugates $f$ to $f^{-1}$. The indeterminacy set consists of the points where $f$ takes the form $\frac{0}{0}$ or $\infty \cdot 0$ and is given by $I(f)=\{(1,0),(-a, \infty)\}$. The critical set is $\mathcal{C}(f)=\{x=1\} \cup\{x=-a\}$, which contains $I(f)$. The critical set for the inverse is $\mathcal{C}\left(f^{-1}\right)=\{y=$ $-1\} \cup\{y=a\}$, and the indeterminacy locus is $I\left(f^{-1}\right)=\{(0,-1),(\infty, a)\}$, as is seen by applying the involution to $\mathcal{C}(f)$ and $I(f) . \quad f$ is smooth on $\overline{\mathbf{R}^{2}}-I(f)$, and $f: \overline{\mathbf{R}^{2}}-\mathcal{C}(f) \rightarrow \overline{\mathbf{R}^{2}}-\mathcal{C}\left(f^{-1}\right)$ is a diffeomorphism.

A calculation shows that $f$ preserves the meromorphic two form

$$
\zeta=\frac{d x \wedge d y}{y-x+1}
$$

This form has no zeroes; it has simple poles along the lines $\{x=\infty\}$, $\{y=\infty\}$, and $\{y-x+1=0\}$. The union of these lines is invariant under $f$. One checks directly that $f$ maps $\{y-x+1=0\}$ onto itself by $(t, t-1) \mapsto(t+a, t+a-1)$, and that $f$ interchanges $\{x=\infty\}$ and $\{y=\infty\}$ according to $(\infty, y) \mapsto(y, \infty) \mapsto(\infty, y+a-1)$. In particular $f^{2}$ restricts to translations on these three lines. It follows that $D f_{(\infty, \infty)}^{2}=\mathrm{id}$, so $(\infty, \infty)$ is a parabolic fixed point for $f$. This point plays a central role in the dynamics of $f$, so we record some information about the behavior of $f$ nearby. 


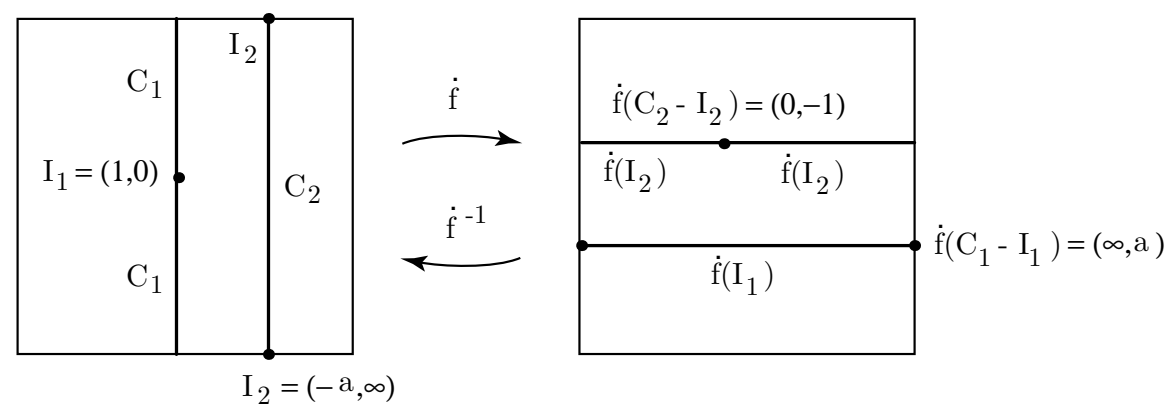

FiguRE 1. Action of $\dot{f}$ and $\dot{f}^{-1}$ on critical sets and points of indeterminacy.

Proposition 1.1. There exists a constant $C>0$ such that $|x|,|y|>C$ implies

$$
\left(\begin{array}{l}
x \\
y
\end{array}\right) \stackrel{f^{2}}{\mapsto}\left(\begin{array}{c}
x\left(1+\frac{a-1}{x}+\frac{a+1}{y}+O\left(|x|^{-2}+|y|^{-2}\right)\right) \\
y\left(1+\frac{a-1}{y}+\frac{a+1}{x}+O\left(|x|^{-2}\right)\right)
\end{array}\right) .
$$

In particular, if we set $m(x, y)=y / x$ (which is negative in $R_{0} \cup R_{1}$ ), then

$$
m \circ f^{2}(x, y)=m(x, y)\left(1-\frac{2}{y}+\frac{2}{x}+O\left(|x|^{-2}+|y|^{-2}\right)\right) .
$$

The proof is a straightforward computation that we leave to the reader.

We say that the parameter $a \in \mathbf{C}$ is exceptional if $I(f) \cap f^{n}\left(I\left(f^{-1}\right)\right) \neq \emptyset$ for some $n \geq 0$. Since both $I(f)$ and $I\left(f^{-1}\right)$ are contained in the three invariant lines, the exceptional values of $a$ are those for which $(-a, \infty)=$ $f^{n}(\infty, a)$ or $(1,0)=f^{n}(0,-1)$. This happens when $a=\frac{n-1}{n+1}$ or $a=\frac{1}{n}$ for some integer $n \geq 1$. Thus no value $a<0$ is exceptional. If $a$ is not exceptional, then $I\left(f^{n}\right)=I(f) \cup \cdots \cup f^{-n+1} I(f)$. We use the notation

$$
I_{+}=\bigcup_{n \geq 0} f^{-n} I(f)=\bigcup_{n \geq 1} I\left(f^{n}\right), \quad I_{-}=\bigcup_{n \geq 0} f^{n} I\left(f^{-1}\right)=\bigcup_{n \geq 1} I\left(f^{-n}\right),
$$

so that $\overline{\mathbf{R}^{2}}-I_{+}$(resp. $\overline{\mathbf{R}^{2}}-I_{-}$) is the set of points where the pointwise forward (resp. backward) dynamics is uniquely defined. Thus $\overline{\mathbf{R}^{2}}-\left(I_{+} \cup I_{-}\right)$ is the set of points which are contained in only one bi-infinite orbit. The largest invariant subset of $\overline{\mathbf{R}^{2}}-\left(I_{+} \cup I_{-}\right)$is

$$
\mathcal{D}_{f}:=\bigcap_{n \in \mathbf{Z}} f^{n}\left(\overline{\mathbf{R}^{2}}-\left(I_{+} \cup I_{-}\right)\right)=\mathbf{R}^{2}-\bigcup_{n \geq 0}\left(f^{n} \mathcal{C}\left(f^{-1}\right) \cup f^{-n} \mathcal{C}(f)\right) .
$$

Thus $\mathcal{D}_{f}$ is the largest set which $f$ maps homeomorphically to itself. $\mathcal{D}_{f}$ is clearly dense in $\overline{\mathbf{R}^{2}}$.

We let $\dot{f}$ denote the closed relation on $\overline{\mathbf{R}^{2}}$ which is obtained by taking the closure of the graph of $f$ restricted to $\overline{\mathbf{R}^{2}}-I(f)$. (We mention Akin [Ak] as a reference for basic material about closed relations.) In other words, $\dot{f}$ is the set-valued mapping given by $\dot{f}(p)=f(p)$ for $p \in \overline{\mathbf{R}^{2}}-I(f), \dot{f}(1,0)=$ $\{y=a\}$, and $\dot{f}(-a, \infty)=\{y=-1\}$. Figure 1 shows how $\dot{f}$ acts on $\overline{\mathbf{R}^{2}}$; $\mathcal{C}(f)-I(f)$ is taken to $I\left(f^{-1}\right)$, and $I(f)$ is taken to $\mathcal{C}\left(f^{-1}\right)$. Since $a$ is not exceptional, the operation of passing to the corresponding closed relation respects the dynamics. That is, $(\dot{f})^{n}=\dot{g}$, where $g=f^{n}$. Let $\dot{f}^{-1}$ denote the closed relation obtained from the restriction of $f^{-1}$ to $\overline{\mathbf{R}^{2}}-I\left(f^{-1}\right)$. With 

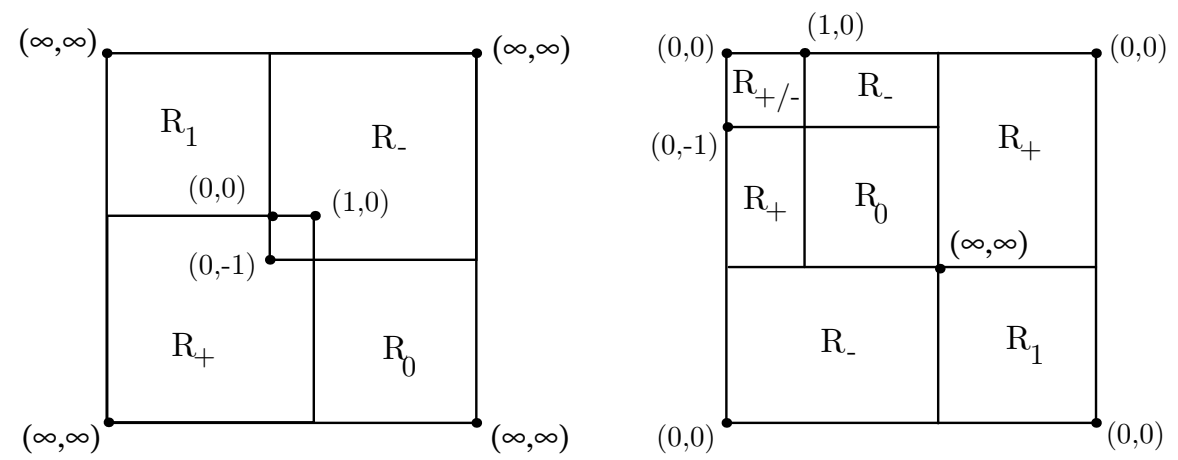

Figure 2. Partition of $\overline{\mathbf{R}^{2}}$ when $a<-1$.

$\mathcal{C}(f)=C_{1} \cup C_{2}$ as in Figure 1 , we see that if $q \in C_{j}$, then $\dot{f}^{-1} \dot{f} q=C_{j}$, for $j=1,2$.

Let $\mathcal{K}$ denote the set of compact subsets of $\overline{\mathbf{R}^{2}}$. The relation $\dot{f}$ induces a map $\dot{f}: \mathcal{K} \rightarrow \mathcal{K}$. Since $\dot{f}$ is a closed relation, it is upper semicontinuous on $\mathcal{K}$. That is, if closed sets $S_{j}$ decrease to $S$, then $\dot{f} S_{j}$ decrease to $\dot{f} S$.

There is a second induced map $f: \mathcal{K} \rightarrow \mathcal{K}$, where $f(S)$ is defined as the closure of $f(S-I(f))$. This map is neither upper or lower semicontinuous. For $S \in \mathcal{K}$ we have

$$
f(S) \subset \dot{f}(S) \subset f(S) \cup \mathcal{C}\left(f^{-1}\right)
$$

Again, since $a$ is not exceptional, the $n$th iterate of $f$ as a map of $\mathcal{K}$ coincides with the mapping of $\mathcal{K}$ induced by $f^{n}$. We also have an induced mapping $f^{-1}: \mathcal{K} \rightarrow \mathcal{K}$. If $S$ is a compact set which is the closure of its interior, then $f(S)$ is also the closure of its interior, and $f^{-1} f(S)=S$.

Consider the covering of $\overline{\mathbf{R}^{2}}$ by closed rectangles:

$$
\begin{aligned}
R_{+} & =[-\infty, 1] \times[-\infty, 0] \\
R_{-} & =[0, \infty] \times[-1, \infty] \\
R_{0} & =[1, \infty] \times[-\infty,-1] \\
R_{1} & =[-\infty, 0] \times[0, \infty] .
\end{aligned}
$$

Two views of this covering are pictured in Figure [2] the right hand illustration is useful for visualizing the fixed point $(\infty, \infty)$, since in a small neighborhood of $(\infty, \infty)$, the action of $f$ is approximately $(s, t) \mapsto(t, s)$, which is a reflection about the diagonal $s=t$.

Proposition 1.2. If $a<-1$ then the following hold:

- $f\left(R_{+}\right) \subset R_{+}$, and if $\left(x_{0}, y_{0}\right) \in R_{+}$and $\left(x_{1}, y_{1}\right)=f\left(x_{0}, y_{0}\right)$, then

$$
\min \left\{x_{1}-1, y_{1}\right\} \leq \min \left\{x_{0}-1, y_{0}\right\}-1 .
$$

- $f^{-1}\left(R_{-}\right) \subset R_{-}$, and if $\left(x_{0}, y_{0}\right) \in R_{-}$and $\left(x_{-1}, y_{-1}\right)=f^{-1}\left(x_{0}, y_{0}\right)$, then

$$
\max \left\{x_{-1}, y_{-1}+1\right\} \geq \max \left\{x_{0}, y_{0}+1\right\}+1 .
$$

- $f\left(R_{1}\right) \cap \operatorname{int} R_{1} \cap \mathbf{R}^{2}=f^{-1}\left(R_{1}\right) \cap \operatorname{int} R_{1} \cap \mathbf{R}^{2}=\emptyset$.

The proof of this proposition is elementary, and we leave its verification to the reader. Taken together, the four conclusions indicate that $f$ has the combinatorial behavior given by the graph on the left side of Figure 3 . For 


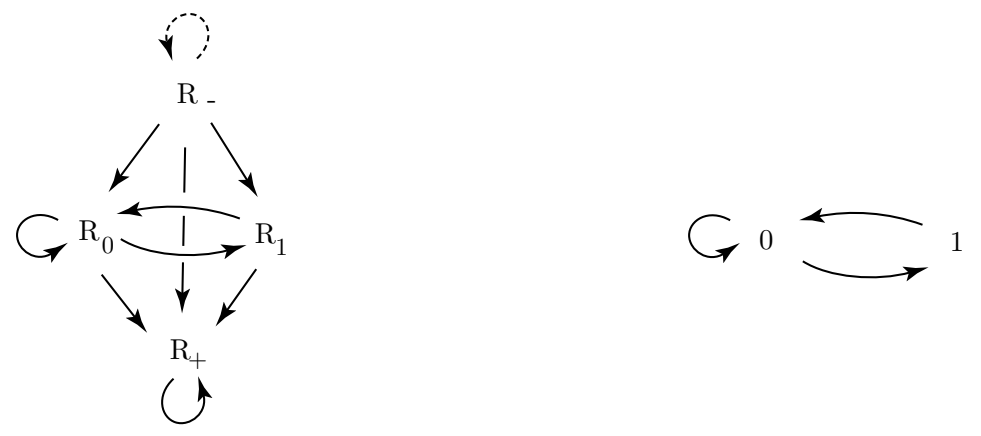

Figure 3. Graph of the Filtration

instance, the arrow from $R_{0}$ to $R_{1}$ indicates that $f\left(R_{0}\right) \cap R_{1}$ contains an open set. The dashed arrow indicates that a point can remain within int $R_{-}$ for only finite positive time. The nontrivial recurrent part of this graph is given by the right hand side of Figure 3 . If we reverse the arrows and move the dashed arrow to $R_{+}$, then we obtain the graphs corresponding to $f^{-1}$.

Let us define the stable set $W^{s}(\infty, \infty)$ as the set of points $p$ such that $f^{n} p \notin I(f)$ for all $n \geq 0$, and $\lim _{n \rightarrow+\infty} \operatorname{dist}\left(f^{n} p,(\infty, \infty)\right)=0$.

Theorem 1.3. We have

$$
W^{s}(\infty, \infty)=\bigcup_{n \geq 0} f^{-n} R_{+}-I_{+} .
$$

In particular, if $f^{n} p \in\left(R_{0} \cup R_{1}\right) \cap \mathbf{R}^{2}$ for all $n \in \mathbf{Z}$, then $f^{n} p$ cannot approach $(\infty, \infty)$ in either forward or backward time.

Proof. If $p \in \bigcup_{n \geq 0} f^{-n} R_{+}-I_{+}$, then we have $\lim _{n \rightarrow \infty} f^{n} p=(\infty, \infty)$ by Proposition 1.2. Conversely, suppose that $p \notin I_{+}$, and $\lim _{n \rightarrow \infty} f^{n} p=$ $(\infty, \infty)$. We will show that $p \in \bigcup_{n \geq 0} f^{-n} R_{+}$. If not, then $f^{n} p \in \operatorname{int}\left(R_{-} \cup\right.$ $R_{0} \cup R_{1}$ ) for all $n \geq 0$; in particular, $f^{n} p \in \mathbf{R}^{2}$. By Proposition 1.2 there can be only a finite interval $0 \leq j \leq J$ for which $f^{j} p \in R_{-}$. Further, by Proposition 1.2 once $f^{j_{0}} p \notin R_{-}$, we must have $f^{j} p \notin R_{-}$for $j \geq j_{0}$. Thus we have $f^{n} p \in R_{0} \cup R_{1}$ for $n \geq N$.

Without loss of generality, we may assume that $f^{2 n} p \in R_{0}$ for all $n \geq 0$. We write $\left(x_{n}, y_{n}\right)=f^{2 n}(p)$ and $m_{n}=y_{n} / x_{n}<0$. Proposition 1.1 gives us

$$
m_{n+1}<m_{n}\left(1-\frac{1}{y_{n}}\right)
$$

so that $\left|m_{n}\right|$ increases with $n$. Therefore Proposition 1.1 also gives us that

$$
y_{n+1} \geq y_{n}-C
$$

for some fixed $C$ and $n$ large enough. From this, we deduce that $\left|y_{n}\right| \leq C n$, that $\sum_{j=0}^{\infty} \frac{1}{y_{n}}$ diverges, and that therefore

$$
\lim _{n \rightarrow \infty} m_{n}=m_{0} \prod_{n=0}^{\infty} \frac{m_{n+1}}{m_{n}}=-\infty .
$$

So for $n$ large enough, we have $m_{n}=y_{n} / x_{n}<-1$ and

$$
x_{n+1}=x_{n}+(a-1) x_{n}+m_{n}(a+1)+O\left(|x|^{-1}+|y|^{-1}\right) \leq x_{n}-1
$$

which contradicts the assumption that $x_{n} \rightarrow \infty$. 
Corollary 1.4. If $p \in W^{s}(\infty, \infty)$, and if $f^{j} p \in R_{0} \cup R_{1}$ for all $j \geq 0$, then we must have $f^{j} p \notin \mathbf{R}^{2}$ for some $j \geq 0$. If in addition $p \in \mathbf{R}^{2}$, then we must have $p \in f^{-n}\{x=1\}$ for some $n \geq 0$.

\section{Coding And Rectangles}

Let us summarize some information about finite subshifts (see $[\mathrm{KH}]$ pages 176-181, and $[\mathrm{LM}])$. We use the symbol space $\mathcal{S}=\{0,1\}^{\mathbf{Z}}$, which consists of bi-infinite sequences $w=\ldots w_{-1} w_{0} \cdot w_{1} w_{2} \ldots$, where $w_{j} \in\{0,1\}$ and the ' $'$ serves to locate the entry with subscript 0 . Let $\sigma: \mathcal{S} \rightarrow \mathcal{S}$ denote the shift operator given by $\sigma(w)=\tilde{w}$, where $\tilde{w}_{j}=w_{j+1}$. We give $\mathcal{S}$ the product space topology, which is generated by the finite cylinder sets $C\left(a_{-N} \ldots a_{N}\right):=\left\{w \in \mathcal{S}: w_{j}=a_{j}\right.$ for $\left.-N \leq j \leq N\right\}$. Thus $\mathcal{S}$ is a compact space homeomorphic to a Cantor set. Let us define $\Sigma$ to be the subspace of $\mathcal{S}$ consisting of all sequences (words) $w=\left(w_{j}\right)_{j \in \mathbf{Z}}$ such that the block "11" appears nowhere in $w$; alternatively, the symbol sequence $\left(w_{j}\right)$ may be generated by following the graph on the right hand side of Figure 3. We refer to $(\sigma, \Sigma)$ as the golden mean subshift.

If $w \in \Sigma$ and if $j \leq k$, we let $w[j, k]:=w_{j} \ldots w_{k}$ denote the $[j, k]$ subword of $w$. We refer to $[j, k]$ as the extent of the word $w[j, k]$. We let $\Sigma^{*}$ denote all the subwords of elements $w \in \Sigma$. If $w \in \Sigma^{*}$, is a word of extent $[-n, m]$, with $-n \leq 0 \leq m$, we let $w^{-}=w[-n, 0]$ denote the $[-n, 0]$ subword and $w^{+}=w[0, m]$ denote the $[0, m]$ subword.

We say that a word $w$ is admissible if $w \in \Sigma^{*}$ and if $w$ has extent $[-n, m]$ with $0 \leq m, n \leq \infty$. We will only work with admissible words in the rest of this paper, so we will use "word" to mean "admissible word."

We let $\Sigma^{+}\left(\right.$resp. $\left.\Sigma^{-}\right)$denote the sets of all $[0, \infty]$ words (resp. $[-\infty, 0]$ ) words in $\Sigma^{*}$. We endow both spaces with the product topology. The onesided shift $\sigma^{+}\left(w_{0} \cdot w_{1} \ldots\right)=w_{1} \cdot w_{2} \ldots\left(\operatorname{resp} . \sigma^{-}\left(\ldots w_{-1} w_{0} \cdot\right)=\ldots w_{-2} w_{-1} \cdot\right)$ gives a continuous self-map of $\Sigma^{+}$(resp. $\Sigma^{-}$).

The incidence matrix corresponding to the graph on the right hand side of Figure 3 is $\left(\begin{array}{ll}1 & 1 \\ 1 & 0\end{array}\right)$, and the powers of this matrix satisfy $\left(\begin{array}{ll}1 & 1 \\ 1 & 0\end{array}\right)^{n}=$ $\left(\begin{array}{cc}F_{n+1} & F_{n} \\ F_{n} & F_{n-1}\end{array}\right)$, where $F_{-1}=1, F_{0}=0$, and $F_{n+1}=F_{n}+F_{n-1}$ denote the Fibonacci numbers. For $k, l \in\{0,1\}$, the $(k, l)$ entry of the $n$-th power of this matrix gives the number of words of length $n+1$ starting at $k$ and ending at $l$. For example, if $-n \leq 0 \leq m$, then the number of $[-n, m]$ words that begin and end with ' 0 ' is $F_{n+m+1}$.

A sequence $w \in \Sigma$ satisfies $\sigma^{n} w=w$ if and only if $w_{n+j}=w_{j}$ for all $j \in \mathbf{Z}$. Such a sequence is determined by a finite word $w_{0} \cdot w_{1} \ldots w_{n}$ with $w_{0}=w_{n}$. We use the notation $\bar{v}:=\ldots v \cdot v v \ldots$ with $v=w_{1} \ldots w_{n}$ to express such periodic words. Since there two possibilities, $w_{0}=0$ or $w_{0}=1$, the number of such words is given by

$$
\#\left\{w \in \Sigma: \sigma^{n} w=w\right\}=\operatorname{trace}\left(\begin{array}{ll}
1 & 1 \\
1 & 0
\end{array}\right)^{n}=F_{n+1}+F_{n-1} .
$$

The topological entropy of $\sigma: \Sigma \rightarrow \Sigma$ is the logarithm of the golden mean $\phi=(1+\sqrt{5}) / 2$. Every $\sigma$-invariant probability measure on $\Sigma$ has entropy less than or equal to $\log \phi$. There is a unique $\sigma$-invariant probability measure $\nu$ 

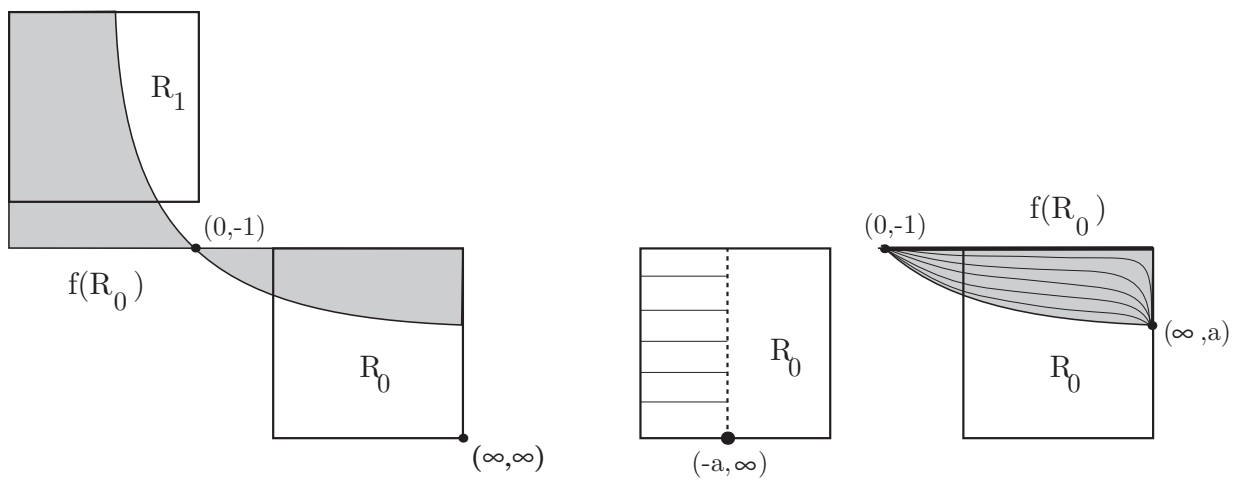

Figure 4. $R(00 \cdot)$

on $\Sigma$ with entropy equal to $\log \phi$. This measure is given by averaging point masses over the periodic points

$$
\nu=\lim _{n \rightarrow \infty} \frac{1}{F_{n+1}+F_{n-1}} \sum_{\sigma^{n} w=w} \delta_{w},
$$

where $\delta_{w}$ denotes the point mass at the point $w$. The measure $\nu$ is also mixing.

There is a unique $\sigma^{+}$-invariant measure $\nu^{+}$on $\Sigma^{+}$with entropy $\log \phi$. The measures $\nu^{ \pm}$are balanced: if $E \subset \Omega^{+}$is a measurable subset and $\left.\sigma^{+}\right|_{E}$ is injective, then $\left.\sigma_{+}^{*} \nu^{+}\right|_{E}=\left.\phi^{-1} \nu^{+}\right|_{\sigma^{+}(E)}$. We may identify $\nu$ with $\nu^{+} \otimes \nu^{-}$ via the product structure

$$
\Sigma \ni v \mapsto\left\{\left(v^{+}, v^{-}\right) \in \Sigma^{+} \times \Sigma^{-}: v^{+}[0]=v^{-}[0]\right\} .
$$

The stable manifold of a point $w \in \Sigma$ is

$$
W^{s}(w)=\{v \in \Sigma: v[n, \infty]=w[n, \infty] \text { for some } n \in \mathbf{N}\} .
$$

We define the local stable manifold

$$
W_{l o c}^{s}(w)=\left\{v \in \Sigma: v^{+}=w^{+}\right\},
$$

which is the cylinder $C\left(w^{+}\right)$over the semi-infinite word $w^{+}$, and it follows that $W^{s}(w)=\bigcup_{n \geq 0} \sigma^{-n} W_{l o c}^{s}(w)$. It is evident that $W_{l o c}^{s}(\tilde{w})=W_{l o c}^{s}(w)$ if and only if $\tilde{w}^{+}=w^{+}$, so that the set of local stable manifolds is parametrized by the set $\Sigma^{+}$, and an individual local stable manifold $W_{l o c}^{s}\left(w^{+}\right)$is parametrized by $\Sigma_{-}$.

For a finite word $w$, we define the rectangle

$$
R(w):=\overline{\bigcap_{k=-n}^{m} f^{-k} \operatorname{int} R_{w_{k}} .}
$$

Since the interiors of $R_{0}$ and $R_{1}$ avoid $I\left(f^{-k}\right)$ for all $k \in \mathbf{Z}$, the definition of $f^{-k}$ is unambiguous. When $w$ is a word of infinite extent, we take $R(w)$ to be the intersection of all rectangles $R\left(w^{\prime}\right)$ corresponding to finite subwords $w^{\prime}$ of $w$. This definition of rectangle will be shown in Theorem 4.9 to essentially coincide with two other plausible definitions of rectangle.

Let us consider an example when $a=-2$. The left hand part of Figure 4 shows $R_{0}, R_{1}$, and $f\left(R_{0}\right)$. The two squares on the right of Figure 4 show how $f$ maps part of $R_{0}$ onto $R(00 \cdot)$, which is the intersection of the shaded 
region with $R_{0}$. The dashed vertical segment inside $\{x=-a\}$ is mapped to the point $(0,-1)$. The top segment in the boundary of $R_{0}$ is mapped to the curved portion of the boundary of $f\left(R_{0}\right)$. The five horizontal segments $\{y=$ const, $1 \leq x \leq-a\}$ in the interior of $R_{0}$, are mapped to the curves connecting $(0,-1)$ and $(\infty, a)$ through the interior of $f\left(R_{0}\right)$. The point of indeterminacy $(a, \infty)$ is mapped to the line $\{y=-1\}$, part of which forms the upper boundary of $R_{0}$. This Figure will be discussed further in connection with Figure 6.

The second item in the following Proposition shows why we assume that all words are admissible.

Proposition 2.1. Let $w$ be a finite $[-n, m]$ word, and let $R(w)$ be the corresponding rectangle. Then

- $R(w)=\overline{\operatorname{int} R(w)}$;

- If $w$ is not admissible, then $R(w)=\emptyset$;

- $f^{k} R(w)=R\left(\sigma^{k} w\right)$ for all $-n \leq k \leq m$;

- $\operatorname{int} R(w) \cap \mathcal{C}\left(f^{k}\right)=\emptyset$ for $-n \leq k \leq m$.

- $p \in \operatorname{int} R(w)$ if and only if $f^{k} p \in \operatorname{int} R_{w_{k}}$ for $-n \leq k \leq m$.

- For all $-n \leq k \leq m, f^{k}$ maps $\operatorname{int} R(w)$ homeomorphically onto int $R\left(\sigma^{k} w\right)$.

Proof. The first conclusion follows because $R(w)$ is the closure of an open set. The second follows from Proposition [1.2 The third results from our convention for images of closed sets under $f$.

To see that the fourth conclusion holds, suppose that it fails for some smallest $k>0$ (the case $k<0$ is similar). Then there exists $p \in \operatorname{int} R(w)$ such that $f^{k-1}(p) \subset \mathcal{C}(f) \cap \operatorname{int} R_{w_{k-1}}$. But this means that $f^{k-1}(p) \in\{x=$ $-a\}$, and $f^{k}(p)=(0,-1) \notin R_{w_{k}}$, which conflicts with the assumption that $p \in R(w) \subset f^{-k} R_{w_{k}}$. Hence the fourth conclusion is true.

We also have that int $R(w) \subset \operatorname{int} R_{w_{0}}$ avoids the indeterminacy set of every iterate of $f$. So in light of the fourth conclusion, the restriction $\left.f^{k}\right|_{\text {int }} R(w)$ is a homeomorphism for each $-n \leq k \leq m$. The fifth and sixth conclusions follow immediately.

We will call a connected arc $\gamma \subset R_{0}$ an $s$-arc if it joins the boundary components $\{y=-1\}$ and $\{y=-\infty\}$ and a $u$-arc if it joins $\{x=1\}$ and $\{x=\infty\}$. Similarly, we call $\gamma \subset R_{1}$ an s-arc if it joins $\{x=0\}$ and $\{x=\infty\}$ and a $\mathrm{u}$-arc if it joins $\{y=0\}$ and $\{y=\infty\}$. In any case, let us call $\gamma$ proper if only its endpoints lie on the boundaries of $R_{0}$ and $R_{1}$ and these points are not corners.

Proposition 2.2. Let $\gamma$ be a proper arc in $R_{0} \cup R_{1}$.

- If $\gamma \subset R_{0}$ is a u-arc, then $f(\gamma)$ contains proper $u$-arcs in $R_{0}$ and $R_{1}$.

- If $\gamma \subset R_{1}$ is a u-arc, then $f(\gamma)$ contains a proper u-arc in $R_{0}$.

- If $\gamma \subset R_{0}$ is an s-arc, then $f^{-1}(\gamma)$ contains proper s-arcs in $R_{0}$ and $R_{1}$.

- If $\gamma \subset R_{1}$ is an s-arc, then $f^{-1}(\gamma)$ contains a proper s-arc in $R_{0}$.

Proof. Suppose that $\gamma \subset R_{0}$ is a proper u-arc. By the intermediate value theorem, there is a smallest subarc $\alpha \subset \gamma$ beginning with the left endpoint of $\gamma$ and ending on $\{x=-a\}$. The map $f$ sends the left endpoint of $\alpha$ to the line $(a, \infty)$ and the right endpoint of $\alpha$ to $(0,-1)$. One sees easily that the 

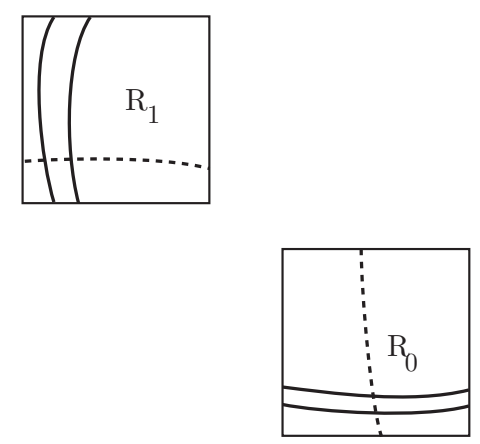

Figure 5. Proper unstable (solid) and stable (dashed) arcs.

intervening points are mapped into the region $(-\infty, 0) \times(-1, \infty)$. Hence $f(\alpha) \cap R_{1}$ contains a proper $\mathrm{u}$-arc. Likewise, there is a smallest subarc $\beta \subset \gamma$ beginning on $x=-a$ and ending with the left endpoint of $\gamma$ on $x=\infty$. The left and right endpoints of $\gamma$ are sent to $(0,-1)$ and $(b, \infty)$, respectively, where $b<-1$. The intervening points are all mapped below $y=-1$, so that $f(\beta) \cap R_{0}$ must contain a proper u-arc. The first assertion is now proved. The proof the second assertion is similar. The last two assertions follow from the reversibility of $f$.

Proposition 2.3. Let $w=w[-n, 0]$ be a finite word, and let $L$ be a horizontal or vertical line that intersects int $R_{w_{-n}}$ in a proper $u$-arc. Then $f^{n}(L) \cap \operatorname{int} R(w)$ contains a proper u-arc $\gamma$. Likewise, if $w=w[0, m]$ is a finite admissible word and $L$ meets $R_{w_{m}}$ in a proper s-arc. Then $f^{-m}(L) \cap R(w)$ contains a proper s-arc $\gamma$.

Proof. We work by induction, considering only the case $w=w[-n, 0]$. By hypothesis $L \cap R_{w_{-n}}$ is a proper u-arc $\gamma_{-n}$. Suppose that for $-n \leq-j<$ $-k \leq 0$ we have proper $u$-arcs $\gamma_{j} \subset R_{w_{-j}}$ satisfying $\gamma_{j-1} \subset f \gamma_{j}$. Then since $w$ is admissible, Proposition 2.2 gives us a proper u-arc $\gamma_{k} \subset f \gamma_{k+1} \cap R_{w_{k+1}}$. Hence $\gamma_{j}$ exists for $0 \leq j \leq n$. Moreover, if $p \in \gamma_{0}$ is not an endpoint, then neither is $f^{-j}(p) \in \gamma_{j}$ for any $j$. Hence the portion $f^{-n}(p), \ldots, p$ of the orbit of $p$ lies entirely in int $R_{0} \cup \operatorname{int} R_{1}$. It follows that $p \in \operatorname{int} R(w)$. Hence $\gamma=\gamma_{0}$ is the arc we are seeking.

Theorem 2.4. $R(w)$ is nonempty.

Proof. Since rectangles corresponding to finite words are compact, and rectangles corresponding to infinite words are decreasing intersections of these, it is enough to prove the proposition for a finite word $w$ of arbitrary extent $[-n, m]$. To do this, we apply the previous proposition to obtain a proper $\mathrm{u}$-arc $\gamma^{-} \subset R\left(w^{-}\right)$and a proper s-arc $\gamma^{+} \subset R\left(w^{+}\right)$. Then $\gamma^{-}, \gamma^{+} \subset R_{w_{0}}$ must meet at some point $p$ that is not an endpoint of either arc. It follows that $p \in \operatorname{int} R\left(w^{+}\right) \cap \operatorname{int} R\left(w^{-}\right)=\operatorname{int} R(w)$, where the equality holds by the definition of $R(w)$ and the fifth conclusion of Proposition 2.1]

\section{Complexification and Intersection Theory}

We will work with the complexification $\tilde{f}$ of $f$; for simplicity, we drop the tilde. The homology group $H_{2}\left(\mathbf{P}^{1} \times \mathbf{P}^{1}, \mathbf{C}\right)$ is two dimensional. Let us fix 
generators: $\gamma_{1}$, which corresponds to the horizontal (complex) line $\mathbf{P}^{1} \times$ $\left\{y_{0}\right\}$, and $\gamma_{2}$, which corresponds to the vertical (complex) line $\left\{x_{0}\right\} \times \mathbf{P}^{1}$. Let $\left\{\gamma_{1}^{*}, \gamma_{2}^{*}\right\}$ denote the basis of $H^{2}\left(\mathbf{P}^{1} \times \mathbf{P}^{1}, \mathbf{C}\right)$ which is dual to $\left\{\gamma_{1}, \gamma_{2}\right\}$. We may represent $\gamma_{1}^{*}$ as the $(1,1)$ form $\frac{i}{2 \pi}\left(1+|x|^{2}\right)^{-2} d x \wedge d \bar{x}$ and $\gamma_{2}^{*}$ as $\frac{i}{2 \pi}\left(1+|y|^{2}\right)^{-2} d y \wedge d \bar{y}$. The homology classes of the preimages under $f$ are: $f^{-1} \gamma_{2} \sim \gamma_{1}+\gamma_{2}$ and $f^{-1} \gamma_{1}=\gamma_{2}$. Thus the induced pullback map $f^{*}$ on the cohomology group $H^{2}\left(\mathbf{P}^{1} \times \mathbf{P}^{1}\right)$ is given with respect to the basis $\gamma_{1}^{*}, \gamma_{2}^{*}$ as

$$
f^{*}=\left(\begin{array}{ll}
0 & 1 \\
1 & 1
\end{array}\right) \text {. }
$$

In particular, the largest eigenvalue of $f^{*}$ is the golden mean $\phi$. By [DF], the pullback of $f^{n}$ on cohomology, $\left(f^{n}\right)^{*}$, coincides with $\left(f^{*}\right)^{n}$ if $a$ is not exceptional. Then the powers of $f^{*}$ are given by the Fibonacci numbers:

$$
\left(\begin{array}{ll}
0 & 1 \\
1 & 1
\end{array}\right)^{n}=\left(\begin{array}{cc}
F_{n-1} & F_{n} \\
F_{n} & F_{n+1}
\end{array}\right)
$$

So we have $f^{n *} \gamma_{1}^{*}=F_{n-1} \gamma_{1}^{*}+F_{n} \gamma_{2}^{*}$ and $f^{n *} \gamma_{2}^{*}=F_{n} \gamma_{1}^{*}+F_{n+1} \gamma_{2}^{*}$.

Complex algebraic curves $V$ and $W$ in $\mathbf{P}^{1} \times \mathbf{P}^{1}$ define cohomology classes $\{V\}=n_{1} \gamma_{1}^{*}+n_{2} \gamma_{2}^{*}$ and $\{W\}=m_{1} \gamma_{1}^{*}+m_{2} \gamma_{2}^{*}$ in $H^{2}$. We use the notation $V \sim\left[n_{1}, n_{2}\right]$ and $W \sim\left[m_{1}, m_{2}\right]$. The intersection product on $H^{2}$ is defined as

$$
V \cdot W=n_{1} m_{2}+n_{2} m_{1} .
$$

Thus the intersection form

$$
\cdot: H^{2}\left(\mathbf{P}^{1} \times \mathbf{P}^{1}\right) \times H^{2}\left(\mathbf{P}^{1} \times \mathbf{P}^{1}\right) \rightarrow \mathbf{C}
$$

is a quadratic form whose matrix is

$$
\left(\begin{array}{ll}
0 & 1 \\
1 & 0
\end{array}\right)
$$

with respect to the basis $\gamma_{1}^{*}, \gamma_{2}^{*}$. A basic result of intersection theory (see [Fu]) is that if all points of $V \cap W$ are isolated, then the intersection product $V \cdot W$ is equal to the number of intersection points $V \cap W$ counted with multiplicity. Since the curves are complex, the multiplicity of each isolated intersection point is an integer $\geq 1$.

The pushforward $f_{*}=\left(f^{-1}\right)^{*}$ of $f$ acting on $H^{2}\left(\mathbf{P}^{1} \times \mathbf{P}^{1}\right)$ is just the adjoint

$$
\left(\begin{array}{ll}
1 & 1 \\
1 & 0
\end{array}\right)
$$

of $f^{*}$ with respect to this form. Thus $f_{*}^{m} \gamma_{1}^{*}=F_{m+1} \gamma_{1}^{*}+F_{m} \gamma_{2}^{*}$, and $f_{*}^{m} \gamma_{2}^{*}=$ $F_{m} \gamma_{1}^{*}+F_{m-1} \gamma_{2}^{*}$. In the sequel we will use this to compute the number of intersections between $f^{n *} \gamma_{i}$ and $f_{*}^{m} \gamma_{j}$. For instance:

$$
f^{n *} \gamma_{2} \cdot f_{*}^{m} \gamma_{1}=F_{n+1} F_{m+1}+F_{n} F_{m} .
$$

Lemma 3.1. For each $n \in \mathbf{N}$, the periodic points of period $n$ for $f$ are isolated.

Proof. The alternative is that $f^{n}$ fixes some algebraic curve $V \subset \mathbf{P}^{1} \times$ $\mathbf{P}^{1}$ pointwise. But $V$ is homologous to a positive linear combination of horizontal and vertical lines and must therefore intersect the line $\{y=x-1\}$ somewhere. Since $f$ acts by translation on this line with $(\infty, \infty)$ as the sole 
fixed point, we see that $V$ must contain the point $(\infty, \infty)$. This contradicts Proposition 3.2 below.

Given the Lemma, let us describe how to count the periodic points of the complexification of $f$. Let $\Gamma_{f^{n}}$ denote the graph of $f^{n}$ as a subvariety of $\left(\mathbf{P}^{1} \times \mathbf{P}^{1}\right) \times\left(\mathbf{P}^{1} \times \mathbf{P}^{1}\right)$. The periodic points of $f$ are given by intersecting $\Gamma_{f^{n}}$ with the diagonal $\Delta \subset\left(\mathbf{P}^{1} \times \mathbf{P}^{1}\right) \times\left(\mathbf{P}^{1} \times \mathbf{P}^{1}\right)$. Thus we have:

$$
\left\{p \in \mathbf{P}^{1} \times \mathbf{P}^{1}: f^{n} p=p\right\}=\Delta \cap \Gamma_{f^{n}} .
$$

Since $f^{-n}(I(f)) \cap I(f)=\emptyset$ for every $n \in \mathbf{N}$, it follows from the Lefschetz Fixed Point Formula $[\mathrm{Fu}]$ that

$$
\#\left\{p \in \mathbf{P}^{1} \times \mathbf{P}^{1}: f^{n} p=p\right\}=\operatorname{trace}\left(f^{*}\right),
$$

where the periodic points are counted according to their multiplicities, and the trace refers to the action of $f^{*}$ on cohomology in all dimensions. The total cohomology is given by $H^{*}\left(\mathbf{P}^{1} \times \mathbf{P}^{1}\right)=H^{0}+H^{2}+H^{4}$. Now $f^{*}$ acts as the identity on $H^{0}$ and $H^{4}$, both of which have dimension 1 , so we may evaluate the total trace to obtain

$$
\#\left\{p \in \mathbf{P}^{1} \times \mathbf{P}^{1}: f^{n} p=p\right\}=F_{n+1}+F_{n-1}+2 .
$$

We have seen that $(\infty, \infty)$ is the only fixed point of $\mathbf{P}^{1} \times \mathbf{P}^{1}-\mathbf{C}^{2}$. Thus we have

$$
\#\left\{p \in \mathbf{C}^{2}: f^{n} p=p\right\}=F_{n+1}+F_{n-1}+2-m_{(\infty, \infty)},
$$

where $m_{(\infty, \infty)}$ denotes the multiplicity of $(\infty, \infty)$ as a fixed point of $f^{n}$, which is defined as the multiplicity of the intersection of $\Delta$ and $\Gamma_{f^{n}}$ at $(\infty, \infty)$.

Proposition 3.2. For every $n \in \mathbf{Z}, n \neq 0$, the point $(\infty, \infty)$ is an isolated fixed point of $f^{n}$ with multiplicity at least two. When $n$ is even, $m_{(\infty, \infty)}=4$.

Proof. Writing $f$ with respect to the coordinates $(\xi, \eta)=(1 / x, 1 / y)$ and employing Proposition 1.1 gives

$$
f^{2}(\xi, \eta)=(\xi, \eta)+Q(\xi, \eta)+O\left(\|(\xi, \eta)\|^{3}\right),
$$

where $Q(\xi, \eta)=\left(\xi^{2}(1-a)-\xi \eta(1+a), \eta^{2}(1-a)-\eta \xi(1+a)\right)$ is a non-degenerate homogeneous map of degree 2 . Therefore ,

$$
f^{2 n}(\xi, \eta)=(\xi, \eta)+n Q(\xi, \eta)+O\left(\|(\xi, \eta)\|^{3}\right)
$$

for every $n \in \mathbf{Z}$. In particular, there exists $C>0$ and $\epsilon=\epsilon(n)$

$$
\left\|f^{2 n}(\xi, \eta)-(\xi, \eta)\right\| \geq C\|(\xi, \eta)\|^{2}
$$

for every $\|(\xi, \eta)\|<\epsilon$. So $(\xi, \eta)=(0,0)$ is isolated as a fixed point of $f^{2 n}$. Fixed points of $f^{n}$ are also fixed by $f^{2 n}$, so $(\xi, \eta)=(0,0)$ is isolated as a fixed point of any iterate of $f$.

The multiplicity of an isolated fixed point of $f^{n}$ is greater than one exactly when one of the eigenvectors of $D f^{n}$ has eigenvalue one. One can easily check that $(1,1)$ is such an eigenvector for $D f_{(\xi, \eta)=(0,0)}^{n}$. To compute the exact multiplicity in the even case, we need to compute the multiplicity of $(0,0)$ as a solution of $f^{2 n}(\xi, \eta)-(\xi, \eta)=n Q(\xi, \eta)+O\left(\|\left(\xi, \eta \|^{3}\right)=(0,0)\right.$. Because $Q$ is non-degenerate and quadratic, it follows that the multiplicity is four.

It will be seen in $\S 6$ that $m_{(\infty, \infty)}=2$ when $n$ is odd. 


\section{Structure of Rectangles}

Here we study the structure of rectangles $R(w)$ for finite $w$. Essentially, we continue $\S 2$, now incorporating complex intersection theory. The first result (Theorem 4.3) is that if $w$ is finite, then the interior of $R(w)$ has a canonical product structure. This product structure extends to points of $R(w) \cap \mathbf{R}^{2}$ but degenerates at $R(w)-\mathbf{R}^{2}$. Problems with points at infinity lead us to consider the special case of words $w$ which are "alternating." The possibilities for (nonempty) $R(w) \cap \mathbf{R}^{2}$ are given in Theorem 4.7 and Corollary 4.8. Theorem 4.7 then leads to characterizations of $R(w)$ (Theorem 4.9) and of $\Omega:=\bigcup_{w \in \Sigma} R(w)$ (Theorem 4.11).

Theorem 4.1. Let $w_{0}, w_{-n} \in\{0,1\}$ be given, and let $W$ denote the set of $[-n, 0]$ words $w$ beginning with $w_{-n}$ and ending with $w_{0}$. Let $L$ be a horizontal or vertical complex line that meets $R_{w_{-n}}$ in a proper u-arc. Then

$$
f^{n} L \cap R_{w_{0}}=\bigcup_{w \in W} R(w) \cap f^{n} L,
$$

and for each $w \in W, R(w) \cap f^{n} L$ is a proper u-arc. If $w_{0}=0$ (respectively $w_{0}=1$ ) then $R(w) \cap f^{n} L$ can be expressed as the graph of a function over the $x$-axis (respectively, $y$-axis).

Proof. We treat only the representative case $w_{-n}=w_{0}=0$ (in particular $L$ is horizontal); the other cases are similar. By Proposition $2.3 f^{n} L \cap i n t R(w)$ contains a proper u-arc $\gamma_{w}$ for each $w \in W$. By Proposition 2.1, we know that $\gamma_{w} \cap \gamma_{\tilde{w}}=\emptyset$ for distinct words $w, \tilde{w} \in W$. Moreover, the definition of a $\mathrm{u}$-arc in $R_{0}$ implies that $\gamma_{w}$ intersects the vertical line $\left\{x=x_{0}\right\}$, for each $x_{0} \in[1, \infty]$ and each $w \in W$. Hence if $x_{0} \in(1, \infty)$, there are at least $F_{n+1}$ distinct intersections between $\left\{x=x_{0}\right\}$ and $f^{n} L$. On the other hand, as complex curves $\left\{x=x_{0}\right\} \cdot f^{n} L=F_{n+1}$, too. Hence there are no further intersections between $\left\{x=x_{0}\right\}$ and $f^{n} L$. Since vertical lines foliate $R_{0}$, we see that $f^{n} L \cap R_{0}=\bigcup_{w \in W} \gamma_{w}$ and that $f^{n} L \cap R(w)$ is a graph over the $x$-axis, as desired.

Let us define projections $\pi_{j}^{s / u}: \mathbf{P}^{1} \times \mathbf{P}^{1} \rightarrow \mathbf{P}^{1}$ for $j=0,1$ according to the formulas

$$
\pi_{0}^{s}(x, y)=\pi_{1}^{u}(x, y)=x, \quad \pi_{0}^{u}(x, y)=\pi_{1}^{s}(x, y)=y .
$$

We choose intervals

$$
T_{0}^{u}=[-\infty,-1], \quad T_{0}^{s}=[1, \infty], \quad T_{1}^{s}=[0, \infty], \quad T_{1}^{u}=[-\infty, 0]
$$

so that for $j=0,1$, the map $\pi_{j}:=\left(\pi_{j}^{s}, \pi_{j}^{u}\right): \mathbf{P}^{1} \times \mathbf{P}^{1} \rightarrow \mathbf{P}^{1} \times \mathbf{P}^{1}$ is a biholomorphism that restricts to a homeomorphism from $R_{j}$ onto $T_{j}^{s} \times T_{j}^{u}$.

More generally, if $w=w[-n, m]$ is a finite word, let us define $\pi_{w}^{s / u}$ : $\mathbf{P}^{1} \times \mathbf{P}^{1} \rightarrow \mathbf{P}^{1}$ by

$$
\pi_{w}^{s}=\pi_{w_{m}}^{s} \circ f^{m}, \quad \pi_{w}^{u}=\pi_{w_{-n}}^{u} \circ f^{-n},
$$

and set $T_{w}^{s}=T_{w_{m}}^{s}, T_{w}^{u}=T_{w_{-n}}^{u}$. This gives us a meromorphic map $\pi_{w}:=$ $\left(\pi_{w}^{s}, \pi_{w}^{u}\right): \mathbf{P}^{1} \times \mathbf{P}^{1} \rightarrow \mathbf{P}^{1} \times \mathbf{P}^{1}$ whose restriction maps $\operatorname{int} R(w)$ to $\operatorname{int} T_{w}^{s} \times T_{w}^{u}$. Note that $\pi_{w}$ depends only on the first and last digits of $w$. Clearly,

$$
\pi_{\sigma^{k} w} \circ f^{k}=\pi_{w}
$$


for $-n \leq k \leq m$. Furthermore, for each $t \in T_{w_{-n}}^{u}$ we have from Theorem 4.1 that $R(w) \cap\left(\pi_{w}^{u}\right)^{-1} t$ is the intersection of $R(w)$ with a proper u-arc. Likewise for each $t \in T_{w_{m}}^{s}$, the fiber $R(w) \cap\left(\pi_{w}^{s}\right)^{-1} t$ is the intersection of $R(w)$ with a proper s-arc. We refer to these fibers as canonical $s / u$-arcs of $R(w)$. Two properties of canonical arcs follow immediately from their definition. We state them only for $\mathrm{u}$-arcs.

- If $\gamma$ is a canonical u-arc of $R(w)$, and if $\sigma w$ is admissible, then $f \gamma$ is a canonical u-arc of $R(\sigma w)$.

- If $\tilde{w}$ extends $w$ to the right, then the canonical u-arcs of $R(\tilde{w})$ are sub-arcs of the canonical $\mathrm{u}$-arcs of $R(w)$.

Where context makes things clear, we will drop the subscripts from $\pi_{w}^{u}, T_{w_{m}}^{u}$, etc.

We say that a curve $V$ belongs to the exceptional locus of $\pi_{w}$ if $\pi_{w}(V)$ is a point.

Proposition 4.2. For any finite word $w=w[-n, m]$, the indeterminacy locus of $\pi_{w}$ is contained in $I\left(f^{-n}\right) \cup I\left(f^{m}\right)$. The intersection between $R(w)$ and the exceptional locus of $\pi_{w}$ is contained in $\pi_{w}^{-1}(\infty, \infty) \cap(\{x=\infty\} \cup\{y=$ $\infty\})$.

Proof. The assertion about the indeterminacy set is clear from the definition of $\pi$. To see that the claim about the exceptional locus of $\pi$ is true, note that $\pi\left(R(w)-\left(I\left(f^{-n}\right) \cup I\left(f^{m}\right)\right)\right) \subset T^{s} \times T^{u}$. So fix $\left(x_{0}, y_{0}\right) \in T^{s} \times T^{u}$. Then any overlap between $\pi^{-1}\left(x_{0}, y_{0}\right)$ and the exceptional set of $\pi$ is a common component of the complex curves $\left(\pi^{s}\right)^{-1} x_{0}$ and $\left(\pi^{u}\right)^{-1} y_{0}$.

Since $\left(\pi_{w_{m}}^{s}\right)^{-1} x_{0}$ is a line in $R_{0} \cup R_{1} \cup R_{-}$, it follows from Proposition 1.2 that any irreducible component of $\left(\pi^{s}\right)^{-1} x_{0}=f^{-m}\left(\pi_{w_{m}}^{s}\right)^{-1} x_{0}$ not equal to $\{x=\infty\}$ or $\{y=\infty\}$ must contain points in $R_{-}-R_{+}$. Similarly, any non-infinite irreducible component of $\left(\pi^{u}\right)^{-1} y_{0}$ contains points in $R_{+}-R_{-}$. Therefore the only candidates for a common irreducible component $V$ of $\left(\pi^{s}\right)^{-1} x_{0}$ and $\left(\pi^{u}\right)^{-1} y_{0}$ are $\{x=\infty\}$ and $\{y=\infty\}$, and it follows that $f^{k}(V)$ is $\{x=\infty\}$ or $\{y=\infty\}$ for all $k \in \mathbf{Z}$. Since $\pi(V)=\left(\pi^{u}(V), \pi^{s}(V)\right)$ is a single point, we must have $\pi(V)=(\infty, \infty)$.

As the next theorem shows, the restriction of $\pi_{w}$ to int $R(w)$ defines a product structure.

Theorem 4.3. If $w$ is a finite word, then $\pi_{w}$ maps int $R(w)$ homeomorphically onto int $T^{s} \times \operatorname{int} T^{u}$. More generally, $\pi$ is injective on $R(w) \cap \mathbf{R}^{2}$. So if $R(w) \subset \mathbf{R}^{2}$, then $\pi_{w}: R(w) \rightarrow T^{s} \times T^{u}$ is a homeomorphism.

Proof. Let $[-n, m]$ be the extent of $w$. Since $f^{-n} \operatorname{int} R(w) \subset \operatorname{int} R_{w_{-n}}$ and $f^{m}$ int $R(w) \subset R_{w_{m}}$, we have that $\pi$ maps int $R(w)$ into int $\left(T^{s} \times T^{u}\right)$.

Fix a point $\left(x_{0}, y_{0}\right) \in \operatorname{int}\left(T^{s} \times T^{u}\right)$. Then the canonical u-arc $\left(\pi^{u}\right)^{-1} y_{0} \cap$ $R\left(w^{-}\right)$must meet the canonical s-arc $\left(\pi^{s}\right)^{-1} x_{0} \cap R\left(w^{+}\right)$at some point $p_{w} \in$ $\operatorname{int} R\left(w^{+}\right) \cap \operatorname{int} R\left(w^{-}\right)=\operatorname{int} R(w)$. So $\pi\left(p_{w}\right)=\left(x_{0}, y_{0}\right)$. That is, $\pi$ is surjective.

On the other hand, $\pi$ depends only on the extent $[-n, m]$ of $w$ and the first and last digits $w_{-n}, w_{m}$. Hence our argument produces a distinct preimage of $\left(x_{0}, y_{0}\right)$ in $\operatorname{int} R(\tilde{w})$ for every $[-n, m]$ word $\tilde{w}$ whose first and last digits agree with those of $w$. It is straightforward to verify that regardless of $w_{-n}$ and $w_{m}$, the number of such words is exactly the same as the intersection 
number of $\left(\pi^{u}\right)^{-1} y_{0}$ and $\left(\pi^{s}\right)^{-1} x_{0}$ treated as complex curves. Therefore (as we argued in Theorem 4.1) there is exactly one preimage of $\left(x_{0}, y_{0}\right)$ in int $R(\tilde{w})$ for each $\tilde{w}$ and no other preimages in $\mathbf{P}^{1} \times \mathbf{P}^{1}$. In particular, $\pi$ is injective on $R(w)$.

We also obtain that fibers of the meromorphic map $\pi$ are discrete over points in int $\left(T^{s} \times T^{u}\right)$. They are therefore discrete over a neighborhood $U$ of int $\left(T^{s} \times T^{u}\right)$ in $\mathbf{P}^{1} \times \mathbf{P}^{1}$. Taking $U$ small enough, we see that $\pi^{-1} U$ is a disjoint union of connected components $U(\tilde{w}) \subset R(\tilde{w})$ for each $[-n, m]$ word $\tilde{w}$ whose first and last digits agree with those of $w$. Because the number of such words is exactly the topological degree of $\pi$, we see that $\pi$ sends $U(\tilde{w})$ holomorphically and injectively onto $U$ for each $\tilde{w}$. We conclude that $\pi$ restricts to a homeomorphism from int $R(w)$ onto int $\left(T^{s} \times T^{u}\right)$.

By continuity, we must have that fibers $\pi^{-1}\left(x_{0}, y_{0}\right) \cap R(w)$ of the restricted map are connected even when $\left(x_{0}, y_{0}\right) \in \partial\left(T^{s} \times T^{u}\right)$. In addition, a point $p \in R(w) \cap \mathbf{R}^{2} \cap \pi^{-1}\left(x_{0}, y_{0}\right)$ in the finite part of a fiber must be isolated by Proposition 4.2. Hence $\pi$ is injective on $R(w) \cap \mathbf{R}^{2}$. In particular if $R(w) \subset \mathbf{R}^{2}$, then $\pi$ maps $R(w)$ homeomorphically onto its image in $T^{s} \times T^{u}$. The image is compact and contains int $\left(T^{s} \times T^{u}\right)$ as a dense subset, so it is in fact equal to $T^{s} \times T^{u}$.

We single out an observation from the proof of the preceding theorem as a separate result.

Proposition 4.4. Given $a, b \in\{0,1\}$ let $\pi:=\left(\pi_{a}^{s} f^{m}, \pi_{b} f^{-n}\right)$. Then

$$
\pi^{-1}\left(\operatorname{int}\left(T_{a}^{s} \times T_{b}^{u}\right)\right)=\bigcup_{w} \operatorname{int} R(w)
$$

where the union is taken over all $[-n, m]$ words $w$ with $w_{-n}=b$ and $w_{m}=a$.

Corollary 4.5. $R(w)$ is connected.

Proof. If $w$ is finite, then $R(w)=\overline{\operatorname{int} R(w)}$ is connected by Theorem 4.1. If $w$ is infinite, it is a decreasing intersection of compact, connected sets and must also be connected.

There are exactly two words in $\Sigma, \overline{01}$ and $\overline{10}$, in which which the digits alternate. We call a word alternating if it is a subword of one of these. We now describe the connection between rectangles that contain points of indeterminacy and (partially) alternating words. First an elementary observation.

Lemma 4.6. Suppose $j, k \in\{0,1\}$ are not both 1 , that $p \in R_{j}-\mathbf{R}^{2}-I(f)$, that $f(p) \in R_{k}-\mathbf{R}^{2}$, and that neither point is a corner of $R_{0}$ or $R_{1}$. Then for any small neighborhood $U \ni p$, we have $f(U)$ is a neighborhood of $p$ and that $f\left(U \cap R_{j}\right)=f(U) \cap R_{k}$.

Proof. Since $p \in R_{j}-\mathbf{R}^{2}$ and $p \neq(-a, \infty)$, it follows that $f$ is a local diffeomorphism at $p$. The Lemma follows because $(\mathbf{R} \times\{\infty\}) \cup(\{\infty\} \times \mathbf{R})$ is an invariant set, and locally near $p, f$ maps $R_{j}$ to $R_{k}$.

Theorem 4.7. Let $w$ be $a[-n, m]$ word.

- $(\infty, \infty)$ belongs to $R(w)$ if and only if $w$ is alternating.

- If $p \notin I\left(f^{-n}\right) \cup I\left(f^{m}\right)$ is not $(\infty, \infty)$, then $p \in R(w)$ if and only if $f^{k}(p) \in R_{w_{k}}$ for $-n \leq k \leq m$. 
- $p \in I\left(f^{m}\right)$ belongs to $R(w)$ if and only if $f^{k} p=(-a, \infty)$ for some $0 \leq k<m$, and $w[-n, k]$ is alternating, but $w[-n, k+1]$ is not.

- $p \in I\left(f^{-n}\right)$ belongs to $R(w)$ if and only if $f^{-k} p=(\infty, a)$ for some $0 \leq k<n$, and $w[-k, m]$ is alternating, but $w[-k-1, m]$ is not.

If $w$ and $\tilde{w}$ are distinct $[-n, m]$ words, then $R(w) \cap R(\tilde{w})$ contains at most one point, which is in $I_{+} \cup I_{-}$, and consequently $R(w) \cap R(\tilde{w}) \cap \mathbf{R}^{2}=\emptyset$.

Proof. We need only establish each conclusion in the case where $w$ is finite. The first conclusion holds because points $q \in R_{0}$ near $(\infty, \infty)$ map to points $f(q) \in R_{1}$ near $(\infty, \infty)$.

For the second assertion, let us suppose first that $q \in \operatorname{int} R(w)$. Then by the fifth item in Proposition 2.1, $f^{j} q \in R_{w_{j}}$ for all $-n \leq j \leq m$. Thus $\Rightarrow$ holds in this case. Now $f^{j}$ is continuous at $p$ since $p \notin I\left(f^{-n}\right) \cup I\left(f^{m}\right) \cup$ $\{(\infty, \infty)\}$. If we approximate $p$ by $q \in \bigcap_{j=-n}^{m} f^{-j}$ int $R_{w_{j}}$, we see that $\Rightarrow$ holds by continuity.

For the case $\Leftarrow$ in the second assertion, we consider first the case $p \in \mathbf{R}^{2}$. By Theorem 4.2, $\pi_{w}$ is holomorphic and open at $p$, and we have observed that $\pi_{w}(p) \in T_{w_{m}}^{s} \times T_{w_{-n}}^{u}$. Let us choose points $q_{j} \in \operatorname{int}\left(T_{w_{m}}^{s} \times T_{w_{-n}}^{u}\right)$ converging to $\pi_{w}(p)$, and let us choose preimages $p_{j} \in \pi_{w}^{-1}\left(q_{j}\right)$ which converge to $p$ as $j \rightarrow \infty$. By Theorem 4.4, we may pass to a subsequence so that $p_{j} \in$ $\operatorname{int} R(\tilde{w})$ for some word $\tilde{w}$ starting with $w_{-n}$ and ending with $w_{m}$. Since $R(\tilde{w})$ is closed, we must have $p \in R(\tilde{w})$. It follows by the $\Rightarrow$ case that $f^{j} p \in R_{\tilde{w}_{j}}$. Thus $\tilde{w}=w$.

If $p \notin \mathbf{R}^{2}$, then $f^{j} p \notin \mathbf{R}^{2}$. The $\Leftarrow$ part of the second assertion then follows by the preceding Lemma.

Next we prove the third assertion. Since $p \in I\left(f^{m}\right)$, there exists a unique $k, 0 \leq k<m$ such that $f^{k} p \in I(f)$. In particular $f^{k} p \in I(f) \cap\left(R_{0} \cup R_{1}\right)=$ $(-a, \infty)$. Now if $p \in R(w)$, we may choose a sequence $p_{l} \rightarrow p$ such that $f^{j} p_{l} \in \operatorname{int}\left(R_{w_{j}}\right)$ for all $-n \leq j \leq m$. By continuity, $f_{p_{l}}^{j} \rightarrow f^{j} p$ for all $-n \leq j \leq k$. Note that $f^{k} p \in R_{0}, f^{k-1} p \in R_{1}$, etc., so by the second item in this Proposition, $p \in R(w[-n, k])$, and $w[-n, k]$ is alternating. Although $f$ is not continuous at $(-a, \infty)$, the points $f^{k+1} p_{l}$ must accumulate only on $\dot{f}(-a, \infty) \cap\left(R_{0} \cup R_{1}\right) \subset R_{0} \cap\{y=-1\}$. Thus $f^{k+1} p_{l} \in R_{0}$, and so $w_{k}=w_{k+1}=0$. Thus $w[-n, k+1]$ is not alternating. This proves the $\Rightarrow$ portion of the third assertion.

To prove the $\Leftarrow$ part of the third assertion, define $v=0 \cdot v_{1} \ldots v_{n-k-1}$ by setting $v_{\ell}=w_{k+1+\ell}$. By Proposition [2.3, $f^{m-k-1}\{y=-1\} \cap R(v)$ contains a proper u-arc $\gamma$. Choose $q \in f^{-m+k+1}\left(\gamma \cap \mathbf{R}^{2}\right)$. Since $q \in\{y=-1\} \cap \mathbf{R}^{2}$, we have $f^{-1} q=(-a, \infty)$, and so $f^{-j} q \notin I\left(f^{-1}\right)$ for all $j \geq 0$. Thus we may apply the second item of this Proposition to conclude that $q \in R(\tilde{v})$ with $\tilde{v}=\overline{10} v$; we see that we may concatenate the $\overline{10}$ on the left of $v$ because $f^{-j} q \in R_{w_{-j}}$ for alternating symbols $w_{-j}$. By Proposition 2.1, we have $p \in f^{-k-1} q \in R\left(\sigma^{-k-1} \tilde{v}\right) \subset R(w)$.

The proof of the fourth assertion is similar.

We can now specialize Theorem 4.7 to the case of infinite points.

Corollary 4.8. For any word $w$, we have the following possibilities:

- If $w$ is alternating, then $R(w) \cap\{x=\infty\}$ and $R(w) \cap\{y=\infty\}$ are (possibly degenerate) intervals containing $(\infty, \infty)$. 


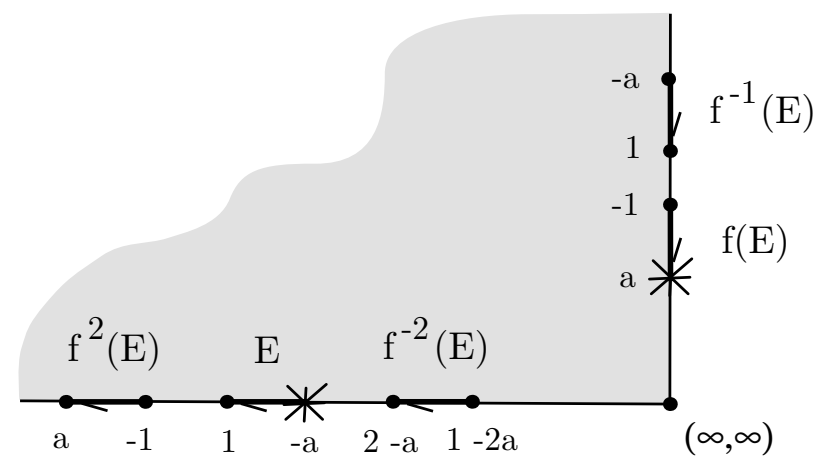

Figure 6 . Orbit of the nontrivial rectangle $R(\overline{10} \cdot \overline{10})=E$

- If $w$ is not alternating, but there exists $n \leq k<m$ such that $w[-n, k]$ and $w[k+1, m]$ are alternating, then $R(w)-\mathbf{R}^{2}$ is the interval $f^{k}(E)$, where

$$
E:=\{(x, \infty): 1 \leq x \leq-a\} .
$$

This case corresponds to $w$ being a subword of a translate of $\overline{10} \cdot \overline{01}$.

- If neither $w^{+}$nor $w^{-}$is alternating, then $R(w) \subset \mathbf{R}^{2}$.

- Otherwise, $R(w)-\mathbf{R}^{2}$ contains exactly one point, and this point belongs to $I\left(f^{-n}\right) \cup I\left(f^{m}\right)$.

Proof. By Theorem 4.7 we know that $p$ belongs to a rectangle $R(w)$ if and only if it is part of an orbit with $w$ as its itinerary. The first assertion of this Corollary is immediate. The third and fourth items of Theorem 4.7 assert that $R(w)$ can contain at most one element of $I\left(f^{-n}\right) \cup I\left(f^{m}\right)$. Further, if there is such an element, then the Theorem says that $w^{+}$or $w^{-}$ is alternating.

It remains to consider points $p \in R(w)-\left(\mathbf{R}^{2} \cup I\left(f^{-n}\right) \cup I\left(f^{m}\right)\right)$. The orbit of such a point will alternate between $R_{0}$ and $R_{1}$ unless there is a $j$ with $f^{j} p \in E$. In this case we have $f^{j} p, f^{j+1} p \in R_{0}$. Note that this can happen for at most one $j$. This completes the proof.

In Figure 6 we have chosen one of the four "quadrants" abutting on $(\infty, \infty)$ to illustrate the interval $E$ and part of its orbit. The "whisker" coming off of $E$ indicates an orientation. The point marked "*" in $E$ indeterminate for $f$; the other " $*$ " is indeterminate for $f^{-1}$. Now recall the right hand side of Figure 4 in which $f(E)$ appears as a vertical boundary segment of $R(00 \cdot)$. Figure 4 also shows some of the canonical $\mathrm{u}$-arcs that foliate $R(00 \cdot)$. The pictures of $R\left((10)^{k} 0 \cdot\right)$ are similar except that as the number of digits increases, the uniform u-arcs get closer to the limiting curve $\{y=-1\}$. The convergence is not uniform because the right endpoint of every canonical $\mathrm{u}$-arc is $(\infty, a)$, regardless of the number of digits. Hence, in the limit, the canonical u-arcs converge to the "L"-shaped rectangle $R(\overline{10} 0 \cdot)=f(E) \cup\left(R_{0} \cap\{y=-1\}\right)$. In light of Corollary 4.8, it will follow from Theorem 6.5 that $E=R(\overline{10} \cdot \overline{01})$.

Theorem 4.9. If $w \in \Sigma^{*}$, the two expressions on the right hand side of

$$
\dot{R}(w):=\bigcap_{k=-n}^{m} f^{-k} R_{w_{k}}=\bigcap_{k=-n}^{m} \dot{f}^{-k} R_{w_{k}}
$$


are equal. Further, $\dot{R}(w)=R(w)$ if $w$ is alternating, and if $w$ is not alternating, we have $\dot{R}(w)=R(w) \cup\{(\infty, \infty)\}$ and $R(w)=\dot{R}(w)-\{(\infty, \infty)\}$. In particular, $R(w)=R\left(w^{+}\right) \cap R\left(w^{-}\right)$.

Proof. Let us start with the observation:

$$
\dot{f}\left(R_{0}\right)-f\left(R_{0}\right)=\{y=-1\}-R_{0} \subset \operatorname{int} R_{+} .
$$

Thus

$$
\left(R_{0} \cup R_{1}\right) \cap \bigcap_{k=-n}^{-1} \dot{f}^{-k} R_{w_{k}}=\left(R_{0} \cup R_{1}\right) \cap \bigcap_{k=-n}^{-1} f^{-k} R_{w_{k}},
$$

from which we deduce that the definition of $\dot{R}(w)$ is unambiguous. Next we note that $(\infty, \infty)$ belongs to $R(w)$ if and only if $w$ is alternating. Finally, consider a point $p \in \overline{\mathbf{R}^{2}}-(\infty, \infty)$. If the orbit of $p$ is disjoint from the indeterminacy set, then $p \in R(w)$ if and only if $f^{j} p \in R_{w_{j}}$ for all $j \in \mathbf{Z}$. Thus $p \in R(w)$ if and only if $p \in \dot{R}(w)$. Otherwise, we may assume that $f^{j} p \in I(f) \cap\left(R_{0} \cup R_{1}\right)=(-a, \infty)$ for some $j \geq 0$. This case is handled by considering the various possibilities in Corollary 4.8 .

By the following result, $R$ is essentially a semi-conjugacy from $(\sigma, \Sigma)$ to $(f, \mathcal{K})$.

Theorem 4.10. If $w$ and $\sigma w$ are admissible, and if $(-a, \infty) \notin R(w)$ then $R(\sigma w)=f R(w)$.

We define

$$
\Omega:=\bigcup_{w \in \Sigma} R(w)
$$

\section{Theorem 4.11.}

$$
\Omega=\bigcap_{n \in \mathbf{Z}} f^{n}\left(R_{0} \cup R_{1}\right)
$$

Proof. The inclusion $\subset$ is evident. We will show the reverse containment. For this, it suffices to show that

$$
\bigcup_{w \in \Sigma} R(w) \supset \bigcap_{m, n=0}^{\infty} \bigcup_{v \in \Sigma[-n, m]} R(v)
$$

where $\Sigma[-n, m]$ denotes the set of admissible $[-n, m]$ words. Now we suppose that $p$ belongs to the right hand intersection. Thus for each $n, m$, there is a word $v$ of extent $[-n, m]$ with $p \in R(v)$. Let us suppose first that $p \notin I_{+} \cup I_{-}$. If $n^{\prime}<n^{\prime \prime}$ and $m^{\prime}<m^{\prime \prime}$, and if $v^{\prime}$ and $v^{\prime \prime}$ are the corresponding words, then $v^{\prime \prime}$ extends $v^{\prime}$. Thus there is a word $w \in \Sigma$ of infinite length which is the common extension of all these finite words. It follows that $p \in R(w)$. If $p \in I_{+} \cup I_{-}$, then by Corollary 4.8 we have $p \in R(w)$, where $w$ is a finite subword of $* 0 \overline{01}$. In this case, too, we obtain an infinite word $w \in \Sigma$ with $p \in R(w)$. This gives the reverse containment, which completes the proof. 


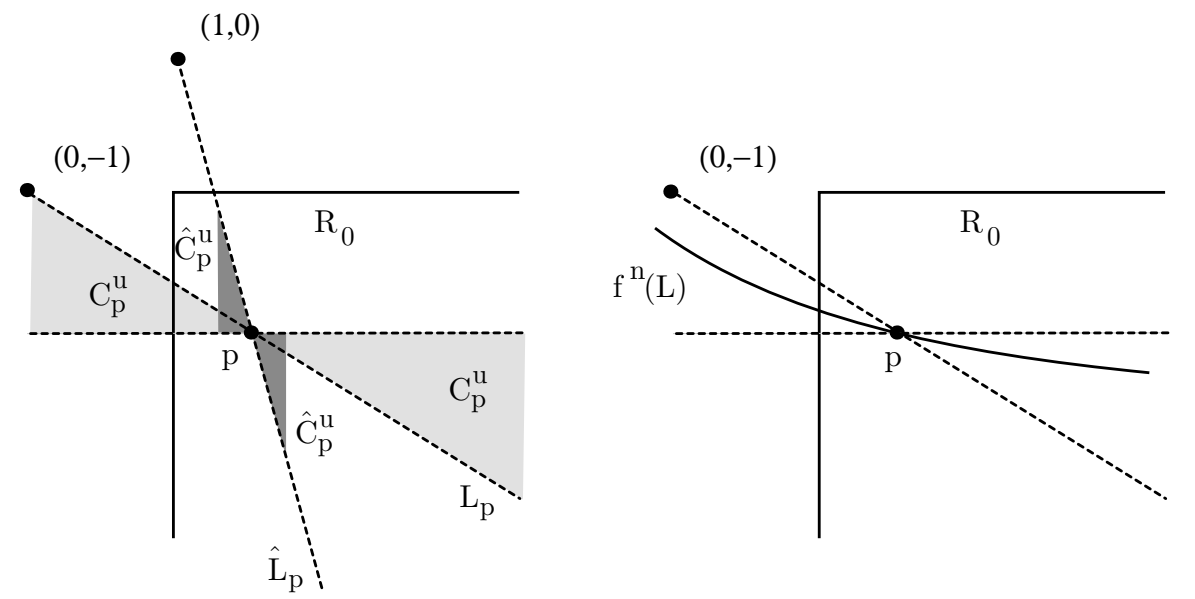

Figure 7. Tangent to $f^{n}(L)$ is between dashed lines.

\section{INVARIANT CONE FIELDS; BOUNDARIES OF RECTANGLES}

In this Section, we show the existence of invariant cone fields for $f$. This allows us to obtain slope bounds for s- and u-arcs. From this we are able to work more effectively with the boundaries of rectangles.

For a point $p \in \mathbf{R}^{2}$, we let $L_{p}$ denote the line from $(0,-1)$ to $p$ and $\hat{L}_{p}$ denote the line from $(1,0)$ to $p$. Let $H_{p}$ and $V_{p}$ denote the horizontal and vertical lines through $p$. For $p \in \mathbf{R}^{2} \cap R_{0}$, we let $\mathcal{C}_{p}^{u}$ denote the cone of tangent vectors $t \in T_{p} \mathbf{R}^{2}$ which are obtained by passing, in the counter-clockwise direction, from $L_{p}$ to $H_{p}$. In other words, $\mathcal{C}_{p}^{u}$ contains those vectors in the second and fourth quadrants between $L_{p}$ and $H_{p}$. The cone $\hat{\mathcal{C}}_{p}^{u}$ is obtained by starting at $\hat{L}_{p}$ and passing in the counter-clockwise direction until we reach $H_{p}$. If $q \in R_{1}$, then we let $\mathcal{C}_{q}^{u}$ (respectively, $\hat{\mathcal{C}}_{q}^{u}$ ) be the cone swept out by starting at $V_{q}$ and moving counter-clockwise until we reach $L_{q}$ (respectively, $\hat{L}_{q}$ ). The cones $\mathcal{C}^{s}$ and $\hat{\mathcal{C}}^{s}$ are obtained as the images of $\mathcal{C}^{u}$ and $\hat{\mathcal{C}}^{u}$ under the involution $(x, y) \mapsto(-y,-x)$. Thus $\mathcal{C}_{p}^{s}$ (respectively, $\hat{\mathcal{C}}_{p}^{s}$ ) is the complement of the interior of $\hat{\mathcal{C}}_{p}^{u}$ (respectively, $\mathcal{C}_{p}^{u}$ ). Figure[7 shows both cones for a point $p \in R_{0}$; the corresponding picture for $p \in R_{1}$ is obtained by reflecting about the line $y=x-1$.

Theorem 5.1. If $p$, fp $\in\left(R_{0} \cup R_{1}\right) \cap \mathbf{R}^{2}$, then the differential $D f_{p}$ maps vectors of $\hat{\mathcal{C}}_{p}^{u}$ to vectors in $\mathcal{C}_{f p}^{u}$.

Proof. Let us assume that $p \in R_{0}$ and fix a vector $t=(1,-\alpha) \in \hat{\mathcal{C}}_{p}^{u}$ for $\alpha>0$. Let $M=\{p+\zeta(1,-\alpha): \zeta \in \mathbf{C}\}$ denote the complex line passing through $p$ in the direction $t=(1,-\alpha)$. With respect to the basis $\left\{\gamma_{1}^{*}, \gamma_{2}^{*}\right\}$ from $\S 3$, the cohomology class $\{M\} \in H^{2}\left(\mathbf{P}^{1} \times \mathbf{P}^{1}\right)$ is the vector $[1,1]$. Likewise, $\{f M\}=f_{*}\{M\}=[2,1]$. We let $H_{f p}$ denote the horizontal line passing through $f p$. Then $\left\{H_{f p}\right\}=[1,0]$, and the intersection multiplicity is

$$
H_{f p} \cdot f M=[1,0] \cdot[2,1]=1 .
$$

It follows that the intersection of $H_{f p}$ and $f M$ at $f p$ is transverse. That is, $D f_{p}(t)$ is not horizontal. 
Similarly, since $L_{f p}$ is neither vertical nor horizontal, we have $\left\{L_{f p}\right\}=$ $[1,1]$, and so

$$
L_{f p} \cdot f M=[1,1] \cdot[2,1]=3 .
$$

Since $M \cap\{x=-a\} \neq \emptyset$, we have $(0,-1) \in f M$. Thus each point of the intersection

$$
\{(0,-1), f p,(\infty, \infty)\} \subset L_{f p} \cap f M
$$

must have multiplicity one, which is to say that each intersection is transverse. Since $\mathcal{C}_{f p}^{u}$ is bounded by the horizontal and $L_{f p}$, we conclude that $D f_{p} t \notin \partial \mathcal{C}_{f p}^{u}$.

We may consider $f M$ as the union of $\operatorname{arcs} \gamma_{+}:=f(M \cap\{x>1\})$ and $\gamma_{-}:=$ $f(M \cap\{x<1\})$. As $t \rightarrow \pm \infty$, we have $f p+t(1,-\alpha)=t(-\alpha, 1)+O(1)$. Thus $f M$ intersects $(\infty, \infty)$ through the second and fourth quadrants. Further, since $t \in \hat{\mathcal{C}}_{p}$, it follows that $M \cap\{x=1\} \subset\{y \leq 0\}$. Thus $\gamma_{+}$begins at $(-\infty,+\infty)$ (in the second quadrant), passes through $(0,-1)$ and then proceeds to $(+\infty, a)$ (i.e. the $y$-coordinate approaches $a$ as $x \rightarrow+\infty)$. We have seen that $L_{f p}$ intersects $\gamma_{+}$transversally, and only in the points $(0,-1)$ and $(\infty, \infty)$. If $f p$ is to the right of the point $(0,-1)$, then the portion of $\gamma_{+}$to the right of $f p$ must be above $L_{f p}$ because $\gamma_{+}$approaches $(a,+\infty)$, whereas $L_{f p}$ approaches $(+\infty,-\infty)$. Thus the tangent to $f M$ lies above the tangent to $L_{f p}$. On the other hand, since the horizontal $H_{f p}$ intersects $\gamma_{+}$only once, the tangent to $f M$ at $f p$ must lie below the horizontal. The two other cases: $f p \in \gamma_{+}$to the left of $(0,-1)$, and $f p \in \gamma_{-}$are handled similarly.

Theorem 5.2. If $p, f p, f^{2} p \in\left(R_{0} \cup R_{1}\right) \cap \mathbf{R}^{2}$, then $D f_{p}^{2}$ maps $\mathcal{C}_{p}^{u}$ strictly inside $\mathcal{C}_{f p}^{u}$.

Proof. Since $\mathcal{C}_{p}^{u} \subset \hat{\mathcal{C}}_{p}^{u}$, it follows from the previous Theorem that $\mathcal{C}_{p}^{u}$ is mapped to $\mathcal{C}_{f p}^{u}$. Now we show that it is mapped strictly inside. Thus, if $t \in$ $\mathcal{C}_{p}^{u}$, we must show that $D f_{p} t \notin \partial \mathcal{C}_{f p}^{u}$. We may assume that $p \in R_{0}$; otherwise, we work with $f p \in R_{0}$ instead. In this case, $\partial \mathcal{C}^{u} \cap \partial \hat{\mathcal{C}}^{u}$ is horizontal, so it suffices to show that if $t$ is horizontal, then $D f_{p}(t)$ is not in $\partial \mathcal{C}_{p}^{u}$. For this, let $H_{p}$ denote the horizontal complex line passing through $p$, with cohomology class $\left\{H_{p}\right\}=[1,0]$. Thus $\left\{f H_{p}\right\}=f_{*}\left\{H_{p}\right\}=[1,1]$, and so

$$
\begin{aligned}
& H_{f p} \cdot f H_{p}=[1,0] \cdot[1,1]=1 \\
& V_{f p} \cdot f H_{p}=[0,1] \cdot[1,1]=1,
\end{aligned}
$$

where $V_{f p}$ denotes the vertical passing through $f p$. It follows that the tangent to $f H_{p}$ is neither horizontal nor vertical at $f p$. Similarly, we have $\{(0,-1), f p\} \subset L_{f p} \cap f H_{p}$, and the intersection number is

$$
L_{f p} \cdot f H_{p}=[1,1] \cdot[1,1]=2 .
$$

Thus the intersection of $L_{f p}$ and $f H_{p}$ is transverse at $f p$, and so the tangent does not belong to the boundary of $\mathcal{C}_{f p}^{u}$. We conclude that $D f_{p}\left(\mathcal{C}_{p}^{u}\right)$ is strictly inside $\mathcal{C}_{f p}^{u}$.

As a consequence, we obtain slope bounds on canonical s- and u-arcs; the first of these slope bounds is illustrated in Figure 7 
Theorem 5.3. Let $L$ be a horizontal or vertical line which meets $R_{0} \cup R_{1}$ in a proper $u$-arc. Let $p \in f^{n} L$ for some $n>0, m$ be the slope of $f^{n} L$ at $p$, and $\tilde{m}$ be the slope of the line joining $p$ to $(0,-1)$.

- If $p \in R_{0}$, then $\tilde{m}<m<0$.

- If $p \in R_{1}$, then $m<\tilde{m}<0$.

Suppose instead that $L$ meets $R_{0} \cup R_{1}$ in a proper s-arc and that $\tilde{m}$ is the slope of the line joining $p \in f^{-n} L$ to $(1,0)$.

- If $p \in R_{0}$, then $m<\tilde{m}<0$.

- If $p \in R_{1}$, then $\tilde{m}<m<0$.

We will call a u-arc $\gamma \subset R_{0}$, uniform if it can be described as the graph $\{(x, g(x))\}$ of a function $g:[1, \infty] \rightarrow[-\infty,-1]$ such that $g \equiv-\infty$, or $g$ is Lipschitz continuous with pointwise derivative $g^{\prime}(x)$ constrained a.e. by the bounds in the first assertion of Theorem 5.3 . We extend the definition of uniformity to u-arcs in $R_{1}$ and s-arcs in $R_{0}$ and $R_{1}$ in the obvious fashion. We say that an arc $\gamma \subset \mathbf{R}^{2}$ is uniform if its closure is uniform. Note, in connection with Figures 4 and 6 , that $R(\overline{10} 0 \cdot) \cap \mathbf{R}^{2}$ is a uniform $\mathrm{u}$-arc. The set $R(\overline{10} 0 \cdot)$, however, is not a uniform $\mathrm{u}$-arc, since it also contains $f^{-1}(E)$.

With this terminology, we may summarize the first two assertions in Theorem 5.3 by saying that for any finite admissible $[-n, 0]$ word $w$, the canonical $\mathrm{u}$-arcs foliating int $R(w)$ are uniform. The following is an easy consequence of the Arzela-Ascoli Theorem.

Proposition 5.4. Let $\left\{\gamma_{j}\right\}_{j \in \mathbf{N}} \subset R_{0}$ be a sequence of uniform u-arcs with graphing functions $g_{j}$. Suppose that $g=\lim _{j \rightarrow \infty} g_{j}$ exists pointwise on $[1, \infty)$. Then the convergence is uniform on compact subsets of $[0, \infty)$, and the limit extends to a function $g:[1, \infty] \rightarrow \mathbf{R}$ whose graph is a uniform $u$-arc in $R_{0}$.

Note that it is not necessarily the case that $g_{j}(\infty) \rightarrow g(\infty)$. This is illustrated on the right hand side of Figure 4, with $g_{j}(\infty)=a$ and $g(\infty)=-1$.

Uniformity of canonical arcs is the key to understanding the boundaries of rectangles.

Theorem 5.5. Let $w \in \Sigma_{-}$be given. Then for every $0 \leq n \leq \infty$ the rectangle $R(w[-n, 0])$ is the set of points in $R_{w_{0}}$ between two uniform u-arcs $\gamma_{1}$ and $\gamma_{2}$. If $n<\infty$, then $\gamma_{1} \cap \gamma_{2} \cap \mathbf{R}^{2}=\emptyset$. If $n=\infty$, then either $\gamma_{1}=\gamma_{2}$, or $R(w)$ has interior.

Proof. For the moment, suppose that $w=w[-n, 0]$ is finite. Then the canonical u-arcs $\left(\pi_{w}^{u}\right)^{-1}(t), t \in T^{u}$, are uniform. Therefore by Proposition 5.4 the two halves of $\partial^{u} R(w)$, which are pointwise limits of canonical u-arcs, are uniform u-arcs. We pointed out earlier that these arcs meet, if at all, in a single infinite endpoint.

Now suppose that $w$ is infinite, and let $\gamma_{1, n}, \gamma_{2, n}$ denote the uniform u-arcs bounding $R(w[-n, 0])$. Because $R(w[-n, 0])$ decreases as $n$ increases, the graphing functions for $\gamma_{1, n}, \gamma_{2, n}$ are monotone in $n$. We apply Proposition 5.4 again as $n \rightarrow \infty$ to extract limiting uniform u-arcs. The convergence of the graphing functions is uniform except at infinity, so we conclude that $R(w) \cap \mathbf{R}^{2}$ is the set of points in $R_{w_{0}} \cap \mathbf{R}^{2}$ between $\gamma_{1}$ and $\gamma_{2}$.

Either $\gamma_{1}$ coincides with $\gamma_{2}$ or the corresponding graphing functions differ at some point. In the first case, $R(w) \cap \mathbf{R}^{2}=\gamma_{1}=\gamma_{2}$. In the second, 
continuity implies that the graphing functions differ on an entire interval. It follows that the region $R(w)$ has interior.

If $w \in \Sigma_{-}, n$, and $\gamma_{1}, \gamma_{2}$ are as in Theorem [5.5] when we set

$$
\partial^{u} R(w[-n, 0])=\gamma_{1} \cup \gamma_{2} .
$$

More generally, we may decompose $\partial R(w)$ into

$$
\partial^{u} R(w)=\partial^{u} R\left(w^{-}\right) \cap R(w), \quad \partial^{s} R(w)=\partial^{s} R\left(w^{+}\right) \cap R(w) .
$$

Since $\partial^{u} R(w)$ is a pair of (not necessarily distinct) uniform $\mathrm{u}$-arcs, it is natural to refer to the intersection of one of these arcs with $R(w)$ as a half of $\partial^{u} R(w)$.

Theorem 5.6. If $w$ is any admissible word, then

$$
\partial R(w)=\partial^{u} R(w) \cup \partial^{s} R(w) .
$$

Each half of $\partial^{u} R(w)$ is connected and meets each half of $\partial^{s} R(w)$ in exactly one point. If $\sigma w$ is well-defined and $R(w), R(\sigma w) \subset \mathbf{R}^{2}$, then

$$
f \partial^{u} R(w)=\partial^{u} R(\sigma w), \quad f \partial^{s} R(w)=\partial^{s} R(\sigma w) .
$$

Proof. The first conclusion is immediate from the fact that $R(w)=R\left(w^{+}\right) \cap$ $R\left(w^{-}\right)$. Because of the bounds on slopes, a uniform u-arc in $R_{w_{0}}$ meets a uniform s-arc in $R_{w_{0}}$ in exactly one point. Therefore, the second conclusion also follows. Finally, if $R(w), R(\sigma w) \subset \mathbf{R}^{2}$, then $f$ maps $\partial R(w)$ homeomorphically onto $\partial R(\sigma w)$. Moreover if $w$ is finite, the pair of proper $\mathrm{u}$-arcs that make up $\partial^{u} R(w)$ must map to proper u-arcs. Hence $f \partial^{u} R(w) \subset \partial^{u} R(\sigma w)$. Likewise, $f^{-1} \partial^{s} R(\sigma w) \subset \partial^{s} R(w)$. This justifies the last conclusion for $w$ of finite extent. A limiting argument justifies it for words of infinite extent.

We refer to the (at most four) points in $\partial^{u} R(w) \cap \partial^{s} R(w)$ as corners of $R(w)$. We denote the corner closest to the origin by $\delta R(w)$ and the corner furthest from the origin by $\tilde{\delta} R(w)$. Since uniform arcs of either type are graphs of non-increasing functions, we see that $\delta R(w)$ is also the corner nearest to the $x$-axis and to the $y$-axis and that $\tilde{\delta} R(w)$ is likewise furthest from either axis. Finally, if $w$ is a $[-n, m]$ word, we have $\delta R(w) \neq \tilde{\delta} R(w)$ unless $n=m=\infty$. When $n$ and $m$ are both infinite, $\delta R(w)=\tilde{\delta} R(w)$ if and only if $R(w)$ is a single point.

\section{PERIODIC POINTS}

In this section we show that if $w \in \Sigma$ is periodic, then $R(w)$ consists of a single periodic point (Theorem 6.3). Further, the correspondence $w \mapsto$ $p \in R(w)$ is essentially a bijection between periodic points of $\sigma$ and periodic points of $f$. Finally, by Theorem 6.4 , all periodic points except $(\infty, \infty)$ are of saddle type.

The alternating words $w \in \Sigma$ are special, as is the parabolic fixed point $(\infty, \infty)$. So we remove them from our discussion of fixed points and define

$$
\operatorname{Fix}^{\prime}\left(\sigma^{n}\right)=\left\{w \in \Sigma: \sigma^{n} w=w\right\}-\{\overline{01}, \overline{10}\}
$$

and

$$
\operatorname{Fix}^{\prime}\left(f^{n}\right)=\operatorname{Fix}\left(f^{n}\right)-\{(\infty, \infty)\}=\operatorname{Fix}\left(f^{n}\right) \cap \mathbf{R}^{2} .
$$

Proposition 6.1. If $w \in \Sigma$ is alternating, then $R(w)=\{(\infty, \infty)\}$. 
Proof. Since $(x, y) \mapsto(-y,-x)$ conjugates $f$ to $f^{-1}$, the set $R(w)=R\left(w^{+}\right) \cap$ $R\left(w^{-}\right)$is symmetric about the line $y=-x$. Therefore assuming $R(w)$ contains points other than $(\infty, \infty)$, we deduce that the corner $\delta R(w) \neq$ $(\infty, \infty)$ of $R(w)$ opposite $(\infty, \infty)$ lies in $\mathbf{R}^{2}$. By Theorem 4.7 $R(w)$ avoids $I\left(f^{n}\right)$ for all $n \in \mathbf{Z}$. Hence $f^{2} R(w)=R\left(\sigma^{2} w\right)=R(w)$ with corners sent to corners. So because $f^{2}$ preserves $(\infty, \infty)$ it also preserves the opposite corner $\delta R(w)=f^{2} \delta R(w)$. Lefschetz fixed point formula predicts that $f^{2}$ has five fixed points, and Proposition 3.2 shows that $(\infty, \infty)$ accounts for four of these. The point $((1-a) / 2,(a-1) / 2) \in R_{0}$ is fixed by $f$ (and therefore by $\left.f^{2}\right)$ and is thus the fifth fixed point. Thus $\delta R(w)=((1-a) / 2,(a-1) / 2)$. But this cannot be, because by Theorem 4.7. $((1-a) / 2,(a-1) / 2) \in R(w)$ only for $w=\overline{0}$. This contradiction shows that $R(w)=\{(\infty, \infty)\}$.

Lemma 6.2. If $w \in \Sigma$ satisfies $\sigma^{n} w=w$, then $R(w) \cap \mathbf{R}^{2}$ contains a fixed point for $f^{n}$. This point belongs to $\mathrm{Fix}^{\prime}\left(f^{n}\right)$ unless $w$ is alternating.

Proof. By Proposition 6.1 we may suppose that $w$ is not alternating. Hence none of the subwords $v^{-}:=w[-n, 0], v^{+}:=w[0, n]$, or $v:=w[-n, n]$ is alternating. Corollary 4.8 therefore implies that $R(v) \subset \mathbf{R}^{2}$. So we invoke Theorem 4.3 to obtain that $\pi_{w_{0}} \circ \pi_{v}^{-1}$ maps $T_{w_{0}}^{s} \times T_{w_{0}}^{u}$ continuously and injectively into $T_{w_{0}}^{s} \times T_{w_{0}}^{u}$. By Brouwer's Theorem, we obtain a fixed point $q=\pi_{w_{0}} \circ \pi_{v}^{-1}(q) \in T_{w_{0}}^{s} \times T_{w_{0}}^{u}$. The point $p=\pi_{v}^{-1}(q) \cap R(v)=\pi_{w_{0}}^{-1}(q) \cap R_{w_{0}}$ lies in $\mathbf{R}^{2}$. Breaking $\pi_{v}$ and $\pi_{w_{0}}$ into components and taking advantage of the fact that $p \notin I\left(f^{n}\right)$, we see

$$
\pi_{v^{-}}^{u}(p)=\pi_{v}^{u}(p)=\pi_{w_{0}}^{u}(p)=\pi_{v^{+}}^{u}(p), \quad \pi_{v^{+}}^{s}(p)=\pi_{v}^{s}(p)=\pi_{w_{0}}^{s}(p)=\pi_{v^{-}}^{s}(p) .
$$

Therefore,

$$
\pi_{v^{+}}(p)=\pi_{v^{-}}(p)=\pi_{\sigma^{n} v^{-}}\left(f^{n} p\right)=\pi_{v^{+}}\left(f^{n} p\right) .
$$

Since $p \in \mathbf{R}^{2}$, we conclude that $p=f^{n}(p)$. By Theorem 4.7 $p \in R(w)$.

Theorem 6.3. If $w \in \Sigma$ satisfies $\sigma^{n} w=w$, then $R(w)=\{p\}$ is a single point satisfying $f^{n} p=p$. If $w$ is not alternating, $p$ has multiplicity one, and $w$ and $p$ have the same period. Finally, the map $w \mapsto R(w)$ defines a bijection between Fix $^{\prime}\left(\sigma^{n}\right)$ and Fix $^{\prime}\left(f^{n}\right)$.

Proof. If $w$ is alternating, then $R(w)=\{(\infty, \infty)\}$ by Proposition 6.1 Otherwise, $w \in \operatorname{Fix}^{\prime}\left(\sigma^{n}\right)$, and by Lemma 6.2 we may choose a point $p=p(w) \in$ $\operatorname{Fix}^{\prime}\left(f^{n}\right) \subset \mathbf{R}^{2}$. If $w$ and $\tilde{w}$ are distinct words in $\operatorname{Fix}^{\prime}\left(\sigma^{n}\right)$, then Theorem 4.7 implies $p(w) \neq p(\tilde{w})$. In particular \# $\operatorname{Fix}^{\prime}\left(\sigma^{n}\right) \leq \# \operatorname{Fix}^{\prime}\left(f^{n}\right)$.

From the discussion of symbolic dynamics in $\S 2$, we have

$$
\# \operatorname{Fix}^{\prime}\left(\sigma^{n}\right)=\# \operatorname{Fix}\left(\sigma^{n}\right)=F_{n+1}+F_{n-1}
$$

if $n$ is odd and

$$
\# \operatorname{Fix}^{\prime}\left(\sigma^{n}\right)=\# \operatorname{Fix}\left(\sigma^{n}\right)-2=F_{n+1}+F_{n-1}-2
$$

if $n$ is even. We may also count the periodic points of $f$. By Proposition 3.2 and the equation preceding it, we have (ignoring multiplicity on the left hand sides)

when $n$ is odd, and

$$
\# \operatorname{Fix}^{\prime}\left(f^{n}\right) \leq F_{n+1}+F_{n-1}
$$

$$
\# \operatorname{Fix}^{\prime}\left(f^{n}\right) \leq F_{n+1}+F_{n-1}-2
$$


when $n$ is even. In either case, it follows that $\# \operatorname{Fix}^{\prime}\left(\sigma^{n}\right)=\# \operatorname{Fix}^{\prime}\left(f^{n}\right)$, and the correspondence $w \rightarrow p(w)$ is bijective. Further, since the count of fixed points of $f^{n}$ without multiplicity coincides with the count with multiplicity, we conclude that each element of $\operatorname{Fix}^{\prime}\left(f^{n}\right)$ has multiplicity one.

Next suppose that $R(w)$ contains two periodic points $f^{n} p=p$, and $f^{m} \tilde{p}=$ $\tilde{p}$. Then $w \in \operatorname{Fix}^{\prime}\left(\sigma^{n m}\right)$ and $p, \tilde{p} \in \operatorname{Fix}^{\prime}\left(f^{n m}\right) \cap R(w)$, contradicting the previous paragraph. Hence $R(w)$ contains at most one periodic point.

Recall that the period of $w$ is the smallest $n$ for which $\sigma^{n} w=w$. In particular, $p(w) \neq p\left(\sigma^{k} w\right)$ for $0 \leq k<n$, so the period of $w$ divides the period of $p(w)$. Lemma 6.2 implies that the reverse is also true, so that $w$ and $p(w)$ have the same period.

Now we wish to show that $R(w)$ is a point for $w \in \operatorname{Fix}^{\prime}\left(\sigma^{n}\right)$. We have $R\left(\sigma^{k} w\right) \subset \mathbf{R}^{2}$ for every $k \in \mathbf{Z}$. Therefore, from the discussion at the end of Section $5 f^{k}: R(w) \rightarrow R\left(\sigma^{k} w\right)$ is a corner preserving homeomorphism. Since there are at most four corners for $R(w)$, each must be a periodic point for $f$. This implies that $R(w)$ has only one corner, which occurs if and only if $R(w)$ is a point.

A consequence of the proof is that $(\infty, \infty)$ is a fixed point of multiplicity 2 for odd iterates of $f$.

Theorem 6.4. Every finite periodic point for $f$ is of saddle type.

Proof. By Theorem 5.2, every fixed point $p=f^{n}(p)$ is simple. That is, no eigenvalue of $D f^{n}(p)$ can be one. Nor can there be an eigenvalue that is a $k$ th root of unity, since that would mean that $p$ has multiplicity greater than one as a point of period $n k$ for some $k>1$. Since $f$ preserves the area form $\zeta=d x \wedge d y /(y-x+1)$, whose singularities are disjoint from $R_{0}^{\prime} \cup R_{1}^{\prime}$, the product det $D f^{n}(p)$ of the eigenvalues of $D f^{n}$ is exactly one. We conclude that either $p$ is a saddle point, or that $D f^{n}$ is conjugate to an irrational rotation. However, this latter conclusion is inconsistent with the fact that $f$ preserves the cone field $\mathcal{C}_{p}^{u}$.

We say that a word $w \in \Sigma_{+}$is eventually alternating if there exists $k \geq 0$ such that $w[k, \infty]$ is alternating.

Theorem 6.5. If $w \in \Sigma_{+}$is alternating, then $R(w)$ is one of the following

- $R(0 \cdot \overline{10})=\{x=\infty\} \cap R_{0}$;

- $R(1 \cdot \overline{01})=\{y=\infty\} \cap R_{1}$.

If $w$ is eventually alternating and $k \geq 1$ is the minimum number for which $w[k, \infty]$ alternates, then $R(w) \cap \mathbf{R}^{2}$ is a uniform s-arc inside $f^{-k+1}\{x=$ 1) $\subset \mathcal{C}\left(f^{k}\right)$.

Proof. Let us start with the word $w=0 \cdot \overline{10}$. By Corollary $4.8, R(w)-\mathbf{R}^{2}=$ $\{x=\infty\} \cap R_{0}$. If $R(w) \neq\{x=\infty\} \cap R_{0}$, then $\partial^{s} R(w) \cap \mathbf{R}^{2}$ also contains a uniform s-arc $\gamma$. It follows that $\gamma$ contains $(\infty, \infty)$. since $R(w) \cap I\left(f^{k}\right)=\emptyset$ for $k \geq 0$, we have $f^{2}(R(w))=R(w) \cap R(010 \cdot)$. In particular, $f^{2} \gamma \subset \gamma$. By Lemma 3.2, the fixed points of $f^{2}$ are isolated, so we can choose a neighborhood $U$ of $(\infty, \infty)$ so that $f^{2}$ has no fixed points in $U-(\infty, \infty)$. We may assume that $f^{2}(\gamma \cap U) \subset \gamma \cap U$ (the case $f^{-2}(\gamma \cap U) \subset \gamma \cap U$ is similar). Since $f^{2}$ has no fixed points except $(\infty, \infty)$, each $p \in \gamma \cap U$ satisfies $f^{2 m} p \rightarrow(\infty, \infty)$ as $m \rightarrow \infty$. This, however, contradicts Theorem 1.3. Thus $R(w)=\{x=\infty\} \cap R_{0}$. 
If $w$ is eventually alternating, then there is a $k>0$ such that $\left(\sigma^{k} w\right)^{+}$ is alternating. By Theorem 4.7. the image $f^{k}\left(R(w) \cap \mathbf{R}^{2}\right)$ is contained in $R(0 \cdot \overline{10})$ or $R(1 \cdot \overline{01})$, which are intervals at infinity. The only points in $\mathbf{R}^{2}$ that are sent to infinity by $f$ are those in $\{x=1\} \subset \mathcal{C}$.

We say that a word $w \in \Sigma_{+}$is pre-periodic if it is pre-periodic for $\sigma^{+}$.

Theorem 6.6. If $w \in \Sigma_{+}$is pre-periodic but not alternating, then $R(w) \cap \mathbf{R}^{2}$ is a uniform s-arc.

Proof. By Corollary [5.5 it suffices to show that int $R(w)=\emptyset$. Theorem 6.5 allows us to assume that $w$ is not eventually alternating, i.e. the (eventual) period $n$ of $w$ is larger than two. Replacing $w$ with $\sigma^{j} w$ for $j$ large enough, we can assume that $w[-n, \infty]$ is periodic. Therefore neither $w[-n, 0]$ nor $w[0, n]$ alternates, and from Corollary 4.8 we have

$$
R\left(\sigma^{k n} w\right) \subset R(w[-n, n]) \subset \mathbf{R}^{2}
$$

for all $k \in \mathbf{N}$. Moreover, the invariant 2-form $\zeta=(y-x+1)^{-1} d x \wedge d y$ satisfies

$$
C^{-1} d x \wedge d y \leq|\zeta| \leq C d x \wedge d y
$$

on $R(w[-n, n])$. Hence

$$
\text { Area (int } R(w)) \leq C \int_{\operatorname{int} R(w)}|\zeta|=C \int_{\operatorname{int} R\left(\sigma^{k n} w\right)}|\zeta| \leq C \text { Area } R\left(\sigma^{k n} w\right) .
$$

But $R\left(\sigma^{n k} w\right)$ decreases to $R(\tilde{w})$, where $\tilde{w} \in \Sigma$ is the periodic extension of $w[-n, n]$. So by Monotone Convergence and Theorem 6.3] it follows that Area $R\left(\sigma^{n k} w\right)$ decreases to Area $R(\tilde{w})=0$ as $k \rightarrow \infty$. We conclude that Area (int $R(w))=0$ and thus int $R(w)=\emptyset$.

\section{UNIFORM ARCS AND ONE-SIDED WORDS}

The following is one of the main results of this paper.

Theorem 7.1. If $w \in \Sigma_{+}$is not alternating, then $R(w) \cap \mathbf{R}^{2}$ is a uniform s-arc.

When $R(w)-\mathbf{R}^{2}$ is a single point, the conclusion of Theorem 7.1 simplifies to the statement that $R(w)$ is itself a uniform s-arc. The only time when this does not happen is when the block '00' appears exactly once in $w$, in which case $R(w)-\mathbf{R}^{2}=f^{-j} E$ for some $j$, by Corollary 4.8 .

The rest of this section will be devoted to the proof of Theorem 7.1; because of Theorem 6.5] we will assume throughout that $w$ is not pre-periodic. By 45 we know that it is sufficient to show that the area of $R(w)$ is zero. The invariant area form is a useful tool, but it is singular at infinity. So we need to study orbits that accumulate at infinity, and for this we analyze the behavior near the parabolic point. We first characterize the itineraries of points in $\Omega$ with unbounded forward orbits.

Lemma 7.2. Let $w \in \Sigma_{+}$and $K \subset R(w) \cap \mathbf{R}^{2}$ be a given compact set. Then the forward orbit $\left\{f^{n} K\right\}_{n \geq 0}$ is unbounded if and only if $w$ contains arbitrarily long alternating subwords. 
Proof. By Theorem 4.7 $f^{n} K \subset R\left(\sigma^{n} w\right)$. The lemma is therefore a consequence of Theorem 6.5

Recall the invariant 2-form

$$
\zeta=(y-x+1)^{-1} d x \wedge d y .
$$

Theorem 7.3. Let $w \in \Sigma_{+}$be given, and suppose that there is a number $M$ such that any alternating subword of $w$ has length no greater than $M$. Then $R(w)$ is a uniform s-arc.

Proof. Theorem 6.6 allows us to assume that $w$ is not eventually periodic. If $n \geq M+1$, then neither $\left(\sigma^{n} w\right)^{+}$nor $\left(\sigma^{n} w\right)^{-}$is alternating, and so $R\left(\sigma^{n} w\right)$ is a compact subset of $\mathbf{R}^{2}$. By Lemma 7.2 there is a compact set $S \subset \mathbf{R}^{2}$ which contains $R\left(\sigma^{n} w\right)$ for all $n \geq M+1$. Let $C$ be a constant such that $\zeta$ satisfies

$$
C^{-1} d x \wedge d y \leq|\zeta| \leq C d x \wedge d y
$$

on $S \cap\left(R_{0} \cup R_{1}\right)$. So

$$
\left.0<\int_{R\left(\sigma^{M+1} w\right)}|\zeta|=\int_{R\left(\sigma^{n} w\right)}|\zeta| \leq C \text { Area } R\left(\sigma^{n} w\right)\right) \leq C \text { Area } S<\infty
$$

for all $n \geq M+1$.

On the other hand, since $w$ is not eventually periodic, $\left(\sigma^{n} w\right)^{+}$is different for every $n \in \mathbf{N}$. Therefore the rectangles $R\left(\sigma^{n} w\right)$ are mutually disjoint. Since $\bigcup_{n=M+1}^{\infty} R\left(\sigma^{n} w\right) \subset S$, we must have Area $R\left(\sigma^{n} w\right)=0$. It follows that $R\left(\sigma^{n} w\right)$ has no interior for any $n \in \mathbf{N}$. By Corollary [5.5] this is all we need to know.

The final and most delicate part of Theorem 7.1 is

Theorem 7.4. Suppose that $w \in \Sigma_{+}$contains arbitrarily long alternating subwords. Then $R(w)$ is a uniform s-arc.

Proof. For $n \geq n_{0}$ sufficiently large, neither $\left(\sigma^{n} w\right)^{-}$nor $\left(\sigma^{n} w\right)^{+}$alternates. Thus $R\left(\sigma^{n} w\right) \subset \mathbf{R}^{2}$. For every $n \geq n_{0}$, let $\left(x_{n}, y_{n}\right)=\delta R\left(\sigma^{n} w\right)$ and $\left(\tilde{x}_{n}, \tilde{y}_{n}\right)=\tilde{\delta} R\left(\sigma^{n} w\right)$ be the vertices of $R\left(\sigma^{n} w\right)$ which are closest and farthest from the origin in $\mathbf{R}^{2}$.

Lemma 7.5. For every $n \geq n_{0}$, we have

$$
\left|x_{n}-\tilde{x}_{n}\right|\left|y_{n}-\tilde{y}_{n}\right| \geq c\left\|\left(x_{n}, y_{n}\right)\right\| \text {. }
$$

for $c=\int_{R(w)}|\zeta|$.

Proof. We estimate:

$$
\begin{aligned}
& \int_{R(w)}|\zeta|=\int_{f^{n} R(w)}|\zeta|=\int_{R\left(\sigma^{n} w\right)}|\zeta| \\
\leq & \int_{x_{n}}^{\tilde{x}_{n}} \int_{\tilde{y}_{n}}^{y_{n}} \frac{d x d y}{\left\|\left(x_{n}, y_{n}\right)\right\|}=\frac{\left|\tilde{x}_{n}-x_{n}\right|\left|y_{n}-\tilde{y}_{n}\right|}{\left\|\left(x_{n}, y_{n}\right)\right\|} .
\end{aligned}
$$

The inequality follows for two reasons. First, we replace $R\left(\sigma^{n} w\right)$ by the euclidean rectangle with vertices $\delta R\left(\sigma^{n} w\right)$ and $\tilde{\delta} R\left(\sigma^{n} w\right)$. Then we estimate $|\zeta|$ using the inequality $|y-x+1| \geq\left\|\left(x_{n}, y_{n}\right)\right\|$ on $R_{0} \cup R_{1}$. 
Lemma 7.6. There exists a large $M>0$ such that if $\max \left\{x_{n},-y_{n}\right\}>M$ and $\max \left\{x_{n+2},-y_{n+2}\right\}>M$, then

$$
f^{2}\left(x_{n}, y_{n}\right)=\left(x_{n+2}, y_{n+2}\right) \text { and } f^{2}\left(\tilde{x}_{n}, \tilde{y}_{n}\right)=\left(\tilde{x}_{n+2}, \tilde{y}_{n+2}\right) \text {. }
$$

Proof. The coordinates of the rectangle $R\left(\sigma^{n} w\right)$ which are nearest to the origin are $\left(x_{n}, y_{n}\right)$, so it follows that all points of the rectangle are at distance at least $M$ from the origin. Since $f^{2}$ acts by translation on $\{x=\infty\}$ and $\{y=\infty\}, f^{2}$ is approximately a translation on $\{\max \{|x|,|y|\}>M\}$. Since $f^{2}$ maps the rectangle $R\left(\sigma^{n} w\right)$ to $R\left(\sigma^{n+2} w\right)$, since $D f^{2}$ preserves the cone fields $\mathcal{C}^{s / u}$, and since the tangents to the sides of the rectangles lie inside these cone fields, it follows that the nearest and farthest vertices $\delta$ and $\tilde{\delta}$ are preserved.

For each $n \in \mathbf{N}$, we set $m_{n}=y_{n} / x_{n}$ and $\tilde{m}_{n}=\tilde{y}_{n} / \tilde{x}_{n}$.

Lemma 7.7. If $\tilde{M}>0$ is large enough and $\min \left\{x_{n},-y_{n}\right\}>\tilde{M}$, then

- $m_{n+2}<m_{n}<0$ and $\tilde{m}_{n+2}<\tilde{m}_{n}<0$;

- $m_{n}<\tilde{m}_{n}$ implies that $m_{n+2}<\tilde{m}_{n+2}$;

Proof. By the hypothesis, the points $\left(x_{n}, y_{n}\right),\left(\tilde{x}_{n}, \tilde{y}_{n}\right),\left(x_{n+2}, y_{n+2}\right)$, and $\left(\tilde{x}_{n+2}, \tilde{y}_{n+2}\right)$ all belong to $R_{0}$ and are near $(\infty, \infty)$. The points $\left(\tilde{x}_{n}, \tilde{y}_{n}\right)$, $\left(\tilde{x}_{n+2}, \tilde{y}_{n+2}\right)$ therefore have the same properties.

The 'slope function' $m(x, y)=y / x$ is meromorphic on $\mathbf{P}^{1} \times \mathbf{P}^{1}$ with a simple pole along $\{x=\infty\}$ and a simple zero along $\{y=\infty\}$. Since $f^{2}$ preserves both of these sets, $m \circ f^{2}$ also has a simple pole along $\{x=\infty\}$ and a simple zero along $\{y=\infty\}$. The latter function has further zeroes and poles along $\mathcal{C}\left(f^{2}\right)$, but these avoid the point $(\infty, \infty)$. Hence $h(x, y):=$ $\left(m \circ f^{2}\right) / m$ is holomorphic in a neighborhood of $(\infty, \infty)$.

We introduce the change of variables $(x, y):=\varphi(s, t):=(1 / s, 1 / t)$, setting $\left(s_{n}, t_{n}\right)=\varphi\left(x_{n}, y_{n}\right)$ and $\left(\tilde{s}_{n}, \tilde{t}_{n}\right)=\varphi\left(\tilde{x}_{n}, \tilde{t}_{n}\right)$. Then $s_{n}>\tilde{s}_{n}>0$ and $t_{n}<$ $\tilde{t}_{n}<0$, and both points $\left(s_{n}, t_{n}\right),\left(\tilde{s}_{n}, \tilde{t}_{n}\right)$ are near the origin. Moreover, $h \circ \varphi$ is holomorphic near $(0,0)$ and Proposition 1.1 tells us that

$$
h \circ \varphi(s, t)=1+2 s-2 t+O\left(\|(s, t)\|^{2}\right) .
$$

In particular, $h\left(x_{n}, y_{n}\right)=h \circ \varphi\left(s_{n}, t_{n}\right)$ and $h\left(\tilde{x}_{n}, \tilde{y}_{n}\right)=h \circ \varphi\left(\tilde{s}_{n}, \tilde{t}_{n}\right)$ both exceed one. This implies the first assertion of the lemma. Moreover,

$$
h\left(x_{n}, y_{n}\right)=h \circ \varphi\left(s_{n}, t_{n}\right)>h \circ \varphi\left(\tilde{s}_{n}, \tilde{t}_{n}\right)=h\left(\tilde{x}_{n}, \tilde{y}_{n}\right),
$$

which implies the second assertion.

Now we complete the proof of Theorem [7.4 Let $c=\int_{R(w)}|\zeta|$ be the constant from Lemma 7.5. We will show that $c=0$, so $\operatorname{int} R(w)=0$. The theorem will then follow from Corollary 5.5. So let us suppose, to the contrary, that $c>0$. Choose $j_{0}$ such that

$$
j_{0}>\frac{|a|+1}{c} .
$$

Let $\tilde{M}$ be as in Lemma 7.7, and increase $j_{0}$ if necessary to obtain

$$
R\left(0 \cdot(10)^{j}\right) \subset\{\tilde{M}<x\} \text { and } R\left((01)^{j} \cdot 0\right) \subset\{y<-\tilde{M}\}
$$

for $j \geq j_{0}$. The map $f^{2 j_{0}}$ acts as translation by $j_{0}(a-1)$ on the line $\{x=\infty\}$, so we may choose $M$ sufficiently large that the second coordinate 
$\pi_{2} f^{2 j_{0}}$ satisfies

$$
\left|\pi_{2} f^{2 j_{0}}(x, y)-y-j_{0}(a-1)\right| \leq 1
$$

for $(x, y) \in\{M<x, a \leq y \leq-1\}$. Now that we have chosen $M$, we may choose $k_{0} \geq j_{0}$ such that

$$
R\left(0 \cdot(10)^{k_{0} / 2}\right) \subset\{M<x\} \text { and } R\left((01)^{k_{0} / 2} 0 \cdot\right) \subset\{y<-M\} .
$$

The word $w$ contains arbitrarily long alternating subwords but is not eventually alternating, so we may find $K \geq k_{0}$ and $N$ such that $w[N, n+2 K]$ is alternating and such that $w[N-1, N]=00$ and $w[N+2 K, N+2 K+1]=$ 00 .

For convenience of notation, let us suppose that $N=0$. Thus $(x, y) \in$ $R(00 \cdot) \subset\{1 \leq x<\infty, a \leq y \leq-1\}$ and $(x, y) \in R\left(0 \cdot(10)^{K}\right) \subset\{M<x\}$ since $K \geq k_{0}$. By our estimate on the second coordinate of $f^{2 j_{0}}$, we have

$$
a+j_{0}(a-1)-1 \leq y_{2 j_{0}}, \tilde{y}_{2 j_{0}} \leq-1+j_{0}(a-1)=1 .
$$

By Lemma 7.5, we have

$$
\tilde{x}_{2 j_{0}} \geq x_{2 j_{0}}+\frac{c||\left(x_{2 j_{0}}, y_{2 j_{0}}\right)||}{\left|y_{2 j_{0}}-\tilde{y}_{2 j_{0}}\right|} \geq x_{2 j_{0}}+\frac{c}{|a|+1} x_{2 j_{0}} .
$$

Now we estimate the slopes

$$
\begin{gathered}
m_{2 j_{0}}=\frac{y_{2 j_{0}}}{x_{2 j_{0}}} \leq \frac{2 j_{0}(a-1)}{x_{2 j_{0}}} \\
\tilde{m}_{2 j_{0}}=\frac{\tilde{y}_{2 j_{0}}}{\tilde{x}_{2 j_{0}}} \geq \frac{a+j_{0}(1-a)-1}{\tilde{x}_{j_{0}}}>\frac{\left(j_{0}+1\right)(a-1)}{(1+c /(|a|+1)) x_{2 j_{0}}} .
\end{gathered}
$$

By our choice of $j_{0}$, we have $m_{2 j_{0}}<\tilde{m}_{2 j_{0}} \leq 0$. Further, we have

$$
\left(x_{j}, y_{j}\right) \in\{\tilde{M}<\min (x,-y)\}
$$

for $2 j_{0} \leq j \leq 2 K-2 j_{0}$. Thus we may apply Lemma 7.7 to conclude that $m_{j}<\tilde{m}_{j}$ for $2 j_{0} \leq j \leq 2 K-2 j_{0}$.

On the other hand, we could have chosen the point $\left(x_{2 K}, y_{2 K}\right)$ as our starting point. In this case we use $f^{-1}$ instead of $f$ and work backwards. (Passing from $f$ to $f^{-1}$ corresponds to applying the involution $(x, y) \mapsto$ $(-y,-x)$.) Our starting point satisfies $\left(x_{2 K}, y_{2 K}\right) \in R(0 \cdot 0) \subset\{1 \leq x \leq$ $-a, y \leq-1\}$ and $\left(x_{2 K}, y_{2 K}\right) \in R\left((10)^{K} \cdot\right) \subset\{y \leq-M\}$. However, when we perform the corresponding slope estimates, we obtain $m_{2 K-2 j_{0}}>\tilde{m}_{2 K-2 j_{0}}$. From this contradiction we conclude that $c=0$, completing the proof of Theorem 7.4.

\section{Conjugacy with the subshift}

We can now make completely explicit the connection between $f$ and the golden mean subshift. The map $R$ turns out to be very nearly a topological conjugacy. Using this map we then transfer the unique measure of maximal entropy from $\Sigma$ to $\Omega$ and draw a number of conclusions about the dynamics of $f$.

Let

$$
\Sigma^{\prime}=\Sigma-\left(W_{l o c}^{s}(\overline{01}, \overline{10}) \cup W_{l o c}^{u}(\overline{01}, \overline{10})\right)
$$

denote the collection of those words $w$ such that neither $w^{+}$nor $w^{-}$is alternating. 
Theorem 8.1. For each $w \in \Sigma^{\prime}$, the set $R(w)$ consists of a single point, and the assignment $w \mapsto R(w)$ gives a homeomorphism between $\Sigma^{\prime}$ and $\Omega \cap \mathbf{R}^{2}$.

Proof. Since $w^{+}$is not alternating, Theorem 7.1 implies that $R\left(w^{+}\right)$is a uniform s-arc. Similarly, $R\left(w^{-}\right)$is a uniform u-arc. Therefore,

$$
R(w) \cap \mathbf{R}^{2}=R\left(w^{+}\right) \cap R\left(w^{-}\right) \cap \mathbf{R}^{2}
$$

contains a unique point $p$. Since $R(w)$ is connected (Corollary 4.5), we conclude that $R(w)=R(w) \cap \mathbf{R}^{2}=\{p\}$.

If $w \in \Sigma$ and, for example, $w^{+}$is alternating then

$$
R(w) \subset R\left(w^{+}\right) \subset\{x=\infty\} \cup\{y=\infty\}
$$

by Theorem 6.5. Therefore, we have from Corollary 4.9 that

$$
\Omega \cap \mathbf{R}^{2}=\bigcup_{w \in \Sigma^{\prime}} R(w)
$$

In other words, $w \mapsto R(w)$ is surjective. But rectangles corresponding to distinct admissible words intersect only at points outside $\mathbf{R}^{2}$ (Theorem 4.7). So the assignment is also injective.

To see that it is continuous, suppose that a sequence $\left\{w^{j}\right\} \subset \Sigma$ converges to $w \in \Sigma$. Then for each $k$, there exists $j_{0}$ such that $j \geq j_{0}$ implies that $w^{j}[-k, k]=w[-k, k]$. Hence $R\left(w^{j}\right) \subset R(w[-k, k])$. Continuity then follows from the definition of $R(w)$ as the decreasing intersection of the sets $R(w[-k, k])$ as $k \rightarrow \infty$.

Continuity of the inverse map follows from continuity of $f$ away from its indeterminacy set and the fact that $p \in \mathbf{R}^{2}$ belongs to $R(w)$ if and only if $f^{k} p \in R_{w_{k}}$ for all $k \in \mathbf{Z}$.

Corollary 8.2. Saddle periodic points of $f$ are a dense subset of $\Omega \cap \mathbf{R}^{2}$, and $\Omega \cap \mathbf{R}^{2}$ is a totally disconnected and perfect subset of $\mathbf{R}^{2}$.

Proof. It is well-known that $\operatorname{Per}^{\prime}(\sigma)$ is dense in $\Sigma^{\prime}$ and that $\Sigma=\overline{\Sigma^{\prime}}$ is a perfect set. Therefore the theorem follows directly from Theorems 6.3 and 8.1 .

Let us set

$$
\Sigma^{\prime \prime}:=\Sigma-\left(W^{s}(\overline{01}, \overline{10}) \cup W^{u}(\overline{01}, \overline{10})\right)=\bigcap_{n \in \mathbf{Z}} \sigma^{n} \Sigma^{\prime} .
$$

Corollary 8.3. The assignment $w \mapsto R(w)$ defines a topological conjugacy between $\left(\sigma, \Sigma^{\prime \prime}\right)$ and $\left(f, \mathcal{D}_{f} \cap \Omega\right)$.

The following result shows that $f$ is topologically expansive on $\Omega \cap \mathbf{R}^{2}$. (Recall that $f^{2} f$ acts as a translation, and thus is not expansive, on $\overline{\mathbf{R}^{2}}-\mathbf{R}^{2}$.)

Theorem 8.4. There is an $\eta>0$ such that if $p, q \in \Omega \cap \mathbf{R}^{2}$ are distinct points, then $\sup _{n \in \mathbf{Z}} \operatorname{dist}\left(f^{n} p, f^{n} q\right)>\eta$.

Proof. Let dist denote a distance function on $\overline{\mathbf{R}^{2}}$. Fix $\eta>0$ such that $\eta<\operatorname{dist}\left(R_{1}, R_{0} \cap\{1 \leq x \leq-a\}\right)$ and $\eta<\operatorname{dist}\left(R_{1}, R_{0} \cap\{-a \leq y \leq-1\}\right)$. By Theorem 8.1 there are $w, v \in \Sigma^{\prime}$ such that $p=R(w)$ and $q=R(v)$. If $p \neq q$, we must have $v \neq w$. Without loss of generality we may assume that $w_{0} \neq v_{0}$ and thus $w_{0}=0$ and $v_{0}=1$. If $\operatorname{dist}(p, q)<\eta$, and if $p \in R_{0}, q \in R_{1}$, then we must have $p \in R_{0} \cap\{-a<x\}$. Now $f\left(R_{0} \cap\{x>-a\}\right) \cap R_{0}=\emptyset$. It follows 
that $f p \in R_{1}$. And by Proposition 1.2, $f q \in R_{1}$. Thus $w_{1}=1$ and $v_{1}=0$. Again, if $\operatorname{dist}(f p, f q)<\eta$, we must have $f q \in R_{0} \cap\{-a<x\}$. Repeating the previous observation, we conclude that $w^{+}$and $v^{+}$are alternating sequences. A similar argument applied to $f^{-1}$ shows that $w$ and $v$ are alternating. This is a contradiction, since by Theorem 8.1 , we have $w, v \in \Sigma^{\prime}$.

Recall from $\S 2$ the measure $\nu$ on $\Sigma$ of maximal entropy (equal to $\log \phi$ ). This measure puts no mass on the 2 -cycle $\{\overline{01}, \overline{10}\}$. Since $\nu$ is finite and all points of $W^{s / u}(\overline{01}, \overline{10})-\{\overline{01}, \overline{10}\}$ are wandering for the restriction of $\sigma$, it follows that $\nu$ puts no mass on $W^{s / u}(\overline{01}, \overline{10})$. Thus $\Sigma^{\prime \prime}$ is a set of full measure for $\nu$, and it follows that $\mu:=R_{*} \nu$ is a probability measure on $\mathcal{D}_{f} \cap \Omega$ which inherits the key properties of $\nu$ :

Corollary 8.5. The measure $\mu$ does not charge $I(f)$, is $f$-invariant and mixing and has entropy $\log \phi$. Further,

$$
\mu=\lim _{n \rightarrow \infty} \frac{1}{\# F i x^{\prime}\left(f^{n}\right)} \sum_{p \in F i x^{\prime}\left(f^{n}\right)} \delta_{p}=\lim _{n \rightarrow \infty} \frac{1}{\# F i x\left(f^{n}\right)} \sum_{p \in F i x\left(f^{n}\right)} \delta_{p},
$$

so $\mu$ reflects the asymptotic distribution of (saddle) periodic points of $f$.

Proposition 8.6. Let $\lambda$ be a probability measure on $\overline{\mathbf{R}^{2}}$ with the following weak invariance property: For each Borel set $E$ there are sets $E^{\prime} \subset \dot{f}(E)$ and $E^{\prime \prime} \subset \dot{f}^{-1}(E)$ such that $\lambda(E)=\lambda\left(E^{\prime}\right)=\lambda\left(E^{\prime \prime}\right)$. Then $\lambda$ puts no mass on $\overline{\mathbf{R}^{2}}-\mathcal{D}_{f}$.

Proof. Let us recall that

$$
\overline{\mathbf{R}^{2}}-\mathcal{D}_{f}=\bigcup_{n \in \mathbf{Z}} \dot{f}^{n}\left(I(f) \cup I\left(f^{-1}\right)\right) .
$$

First, by the invariance property, $\lambda$ can put no mass on $I(f)$. For if $p \in I(f)$ has positive mass, then $\lambda\left(f^{-n} p\right)=\lambda(p)>0$ for $n \geq 0$. Thus $\lambda$ would have infinite mass, since $f^{-n} p$ is disjoint from $f^{-m} p$ if $n \neq m$. Similarly, $\lambda$ puts no mass on $\bigcup_{n \geq 0}\left(f^{-n} I(f) \cup f^{n} I\left(f^{-1}\right)\right)$.

Finally, consider a Borel subset $E \subset \dot{f}^{N} p$ for $p \in I(f)$. Without loss of generality $E$ is disjoint from $\bigcup_{j \geq 0} f^{j} I\left(f^{-1}\right)$. Thus $f^{-N}$ is smooth on $E$, and $\lambda(E)=\lambda\left(f^{-N} E\right)=\lambda\{p\}=0$.

Any measure $\lambda$ on $\overline{\mathbf{R}^{2}}$ which is $f$-invariant in the sense of the previous Proposition will live on $\mathcal{D}_{f}$. By Theorem $1.2, \lambda$ can put no mass on int $\left(R_{+} \cup\right.$ $\left.R_{-}\right)$. By Theorem 4.11, all of the mass of $\lambda$ is on $\Omega$, and thus $\lambda$ is carried by $\Omega \cap \mathcal{D}_{f}$. Thus it will be of the form $\lambda=R_{*} \eta$ for some $\sigma$-invariant measure $\eta$ on $\Sigma$. From the fact that $\nu$ is the unique measure of maximal entropy on $\Sigma$, we obtain:

Corollary 8.7. $\mu$ is the unique measure of entropy $\geq \log \phi$ on $\overline{\mathbf{R}^{2}}$.

We say that a bi-infinite sequence $\hat{x}=\left(x_{n}\right)_{n \in \mathbf{Z}}$ is an $f$-orbit if $x_{n+1} \in \dot{f} x_{n}$ for all $n \in \mathbf{Z}$. Let $X$ be a compact subset of $\overline{\mathbf{R}^{2}}$. By $\hat{X}_{f}$ we denote the space of $f$-orbits $\hat{x}$ such that $x_{n} \in X$ for all $n \in \mathbf{Z}$. This is a compact subspace of the infinite product space $X^{\mathbf{Z}}$. We let $\hat{f}$ denote the shift map on $\hat{X}_{f}$, which means that $\hat{f} \hat{x}=\hat{y}$, where $\hat{x}=\left(x_{n}\right)$ and $\hat{y}=\left(y_{n}\right)$ are sequences with $y_{n}=x_{n+1}$. It follows that $\hat{f}$ is a homeomorphism of $\hat{X}_{f}$. 
Let $\pi: \hat{X}_{f} \rightarrow X$ be the projection defined by $\pi \hat{x}=x_{0}$. If $x \in \mathcal{D}_{f}$, then $x$ is contained in a unique $f$-orbit $\iota(x):=\left(f^{n} x\right)_{n \in \mathbf{Z}}$. In fact, $\pi: \pi^{-1}\left(\mathcal{D}_{f}\right) \rightarrow \mathcal{D}_{f}$ is a homeomorphism, and its inverse is given by $\iota$. We may use $\iota$ to push $\mu$ up to an invariant measure $\iota_{*} \mu$ on $\iota\left(\mathcal{D}_{f}\right) \subset \widehat{\mathbf{R}^{2}}{ }_{f}$.

Proposition 8.8. If $\lambda$ is an invariant probability measure on $\hat{X}_{f}$ with $X=$ $\overline{\mathbf{R}^{2}}$, then $\lambda$ puts full measure on $\iota\left(\mathcal{D}_{f}\right)$. Thus $\iota_{*} \mu$ is the unique measure of entropy $\log \phi$ on $\iota\left(\mathcal{D}_{f}\right)$.

Proof. Pushing $\lambda$ down to $\overline{\mathbf{R}^{2}}$, we obtain a measure $\pi_{*} \lambda$ which is invariant in the sense of Proposition 8.5. Thus $\pi_{*} \lambda$ puts no mass on the complement of $\mathcal{D}_{f}$. Thus $\lambda$ can put no mass on $\pi^{-1}\left(\overline{\mathbf{R}^{2}}-\mathcal{D}_{f}\right)$.

If $\lambda$ is an invariant measure of entropy $\log \phi$ on $\hat{X}_{f}$, then $\lambda$ lives on $\iota \mathcal{D}_{f}$. Thus we may identify $\lambda$ with an $f$-invariant measure on $\mathcal{D}_{f}$ with entropy $\log \phi$. This measure must be $\mu$, so $\lambda=\iota \mu$

Now we discuss the topological entropy of $f$. The approach we follow here is to replace $f$ by the map $\hat{f}$ acting on the orbit space. In this case, $(\hat{f}, \hat{\Omega})$ is a compactification of the restriction of $f$ to $\mathcal{D}_{f}$. A second approach would be to work directly with $f$, as is done by Guedj [Gu2].

Theorem 8.9. The topological entropy of $\hat{f}$ on $\hat{X}_{f}$ is equal to $\log \phi$ for $X=\Omega, \overline{\mathbf{R}^{2}}$, and $\mathbf{P}^{1} \times \mathbf{P}^{1}$.

Proof. Let us consider first the case $X=\mathbf{P}^{1} \times \mathbf{P}^{1}$. In this case Friedland [Fr] has shown that $h_{\text {top }}\left(f, \hat{X}_{f}\right)$ is bounded above by the logarithm of the spectral radius of $f^{*}$ action on cohomology $H^{*}(X)$. We have seen that $f^{*}$ is represented by the matrix $\left(\begin{array}{ll}1 & 1 \\ 1 & 0\end{array}\right)$, and thus the spectral radius is given by the golden mean $\phi$. It follows that $h_{\text {top }}\left(f, \hat{X}_{f}\right) \leq \log \phi$ for all three choices $X$.

Now we consider the case $X=\Omega$. We have seen that $\iota_{*} \mu$ is an invariant measure on $\hat{X}_{f}$ with entropy equal to $\log \phi$. Since the topological entropy dominates the entropy of any invariant measure, it follows that $h_{\text {top }}\left(f, \hat{X}_{f}\right) \geq$ $\log \phi$. Thus $h_{t o p}\left(f, \hat{X}_{f}\right)=\log \phi$ for all three choices of $X$.

We note that $(\infty, \infty)$ belongs to $\mathcal{D}_{f}$ and is contained in a unique orbit $\iota(\infty, \infty)$, which is the constant sequence $(\infty, \infty)$. Let us write

$$
\hat{\Omega}_{*}:=\hat{\Omega}_{f}-\iota(\infty, \infty)=\pi^{-1}(\Omega-(\infty, \infty)) .
$$

Each $x \in \Omega-(\infty, \infty)$ is contained in $R_{j}$ for a unique $j$. Thus we have a coding map

$$
c: \hat{\Omega}_{*} \rightarrow \Sigma
$$

given by $c(\hat{x})=\left(w_{n}\right)$, where $w_{n}$ is chosen such that $x_{n} \in R_{w_{n}}$ for all $n \in \mathbf{Z}$. It follows that

$$
c:\left(\hat{f}, \hat{\Omega}_{*}\right) \rightarrow(\sigma, \Sigma)
$$

is a semi-conjugacy. This is an inverse to the mapping $R$ in the following sense: if $x \in \mathcal{D}_{f}$ and $\hat{x}=\iota(x)$, then $R(c(\hat{x}))=\{x\}$, which means that $\pi=R \circ c$ as a mapping from $\iota\left(\mathcal{D}_{f}\right)$ to $\mathcal{D}_{f}$. In other words,

Proposition 8.10. For $\hat{x} \in \pi^{-1}\left(\Omega \cap \mathbf{R}^{2}\right)$, we have $\pi(\hat{x})=R(c(\hat{x}))$. More generally, for $\hat{x} \in \hat{\Omega}_{*}$, we have $\pi(\hat{x}) \in R(c(\hat{x}))$. 


\section{Parabolic basin; Nonwandering Set}

In this section and the next, we study the sets

$$
\Omega_{+}=\bigcup_{w \in \Sigma_{+}} R(w), \text { and } \Omega_{-}=\bigcup_{w \in \Sigma_{-}} R(w) .
$$

By Theorem 4.9 we have

$$
\Omega=\Omega_{+} \cap \Omega_{-} .
$$

By Theorem 7.1, each $R(w)$ is a uniform arc, and because of this, the sets $\Omega_{ \pm} \cap \mathbf{R}^{2}$ have product structure. In this section we show that $\Omega_{+}$is the complement of the forward basin $\mathcal{B}_{+}$inside $R_{0} \cup R_{1}$. Then we identify the nonwandering set as the complement of the total basin $\mathcal{B}_{+} \cup \mathcal{B}_{-}$inside $\overline{\mathbf{R}^{2}}$.

We define the forward basin of $(\infty, \infty)$, written $\mathcal{B}_{+}$, to be the set of points $p$ which are contained in neighborhoods $U$ such that $f^{n} \mid U$ converges uniformly to $(\infty, \infty)$ as $n \rightarrow+\infty$.

Theorem 9.1. The forward basin is $\mathcal{B}_{+}=\operatorname{int}\left(W^{s}(\infty, \infty)\right)$.

Proof. Let us start by noting that $I_{+} \cap \mathcal{B}_{+}=\emptyset$. For if $p \in I(f)$, then $\dot{f} p$ is one of the horizontal curves in the right hand side of Figure 1, and for all $j \geq 1 \dot{f}^{j} p$ is a nontrivial element of $\pi_{1}\left(\overline{\mathbf{R}^{2}}\right)$. Thus $\dot{f}^{j} p$ cannot be contained in a disk about $(\infty, \infty)$.

It follows that $\mathcal{B}_{+}$is an open subset of $W^{s}(\infty, \infty)$, so it suffices to show that $\operatorname{int}\left(W^{s}(\infty, \infty)\right)=\mathcal{B}_{+}$. In fact we will show that these sets both coincide with the set of all points of $W^{s}(\infty, \infty)$ whose forward orbits are contained in $\mathbf{R}^{2}$. One direction is clear. For if $p \in W^{s}(\infty, \infty)$, we must have $f^{n} p \in R_{+}$ for some positive $n$. If $f^{n} p \in R_{+} \cap \mathbf{R}^{2}$, then there is a neighborhood $U$ of $p$ such that $f^{n+1} U \subset R_{+}$. Thus $p$ belongs to both int $W^{s}(\infty, \infty)$ and $\mathcal{B}_{+}$.

It remains to consider the case of $p \in W^{s}(\infty, \infty)$ such that $f^{n} p \notin \mathbf{R}^{2}$ for some $n \geq 0$. Let us first claim that there exists a neighborhood $U$ of $p$ such that $f^{k} U \cap R_{0}$ contains a connected open set $V$ such that $\bar{V} \cap\{x=\infty\} \neq \emptyset$. Let $n \geq 0$ be the smallest number such that $f^{n} p \notin \mathbf{R}^{2}$. If $n=0$, then for sufficiently large $k, f^{k} U$ will in fact contain a one-sided neighborhood of $f^{k} p$ inside $R_{0}$. If $n>0$, then $f^{n-1} p \in\{x=1\} \cap \mathbf{R}^{2}$, and $f^{n-1} U$ is a neighborhood of $f^{n-1} p$ in $\mathbf{R}^{2}$. We see that $f^{n+k} U$ will have the desired property.

We will conclude the proof by showing that $V \not \subset W^{s}(\infty, \infty)$ and thus $p$ belongs to neither int $W^{s}(\infty, \infty)$ nor $\mathcal{B}_{+}$. By Theorem $6.5, R\left(\cdot(01)^{j}\right)$ is a small one-sided neighborhood of $\{x=\infty\} \cap R_{0}$ inside $R_{0}$, and this neighborhood shrinks to $\{x=\infty\} \cap R_{0}$ as $j \rightarrow \infty$. Thus we may choose $j$ sufficiently large that any s-arc in $R\left(\cdot(01)^{j}\right)$ crosses $V$. On the other hand, if $w$ is not eventually alternating, we have $R(w) \cap W^{s}(\infty, \infty)=\emptyset$. Since words which are not eventually alternating can begin with $(01)^{j}$, we see that $V \not \subset W^{s}(\infty, \infty)$, as claimed.

To define the backward basin $\mathcal{B}_{-}$, we replace $f$ with $f^{-1}$; the analogue of the theorem above holds for $W^{u}(\infty, \infty)$ and $\mathcal{B}_{-}$.

Each of the sets $\Omega_{+} \cap R_{j} \cap \mathbf{R}^{2}$ carries a natural product structure. To see this, let us define

$$
\mathcal{T}_{j, \pm}=\left\{w \in \Sigma_{ \pm}: w_{0}=j, \text { and } w \text { not alternating }\right\} .
$$


For instance, if $j=0$, we set $I=(-\infty,-1]$, and we have a homeomorphism

$$
\mathcal{T}_{+, 0} \times I \ni\left(w^{+}, t\right) \mapsto R\left(w^{+}\right) \cap\{y=t\} \in \Omega_{+} \cap R_{0} .
$$

To see that this map in fact defines a homeomorphism, note that for each $w^{+} \in \mathcal{T}_{+, 0}, R\left(w^{+}\right) \cap \mathbf{R}^{2}$ is a uniform s-arc in $R_{0}$ which intersects $\{y=$ $t\}$ in a single point. Similarly, if $j=1$, we set $I=[1, \infty)$ and have a homeomorphism

$$
\mathcal{T}_{+, 1} \times I \cong \Omega_{+} \cap R_{1} \cap \mathbf{R}^{2} .
$$

Lemma 9.2. $\Omega_{+}=\left(R_{0} \cup R_{1}\right)-\mathcal{B}_{+}$.

Proof. If $p \notin \Omega_{+}$, then by Proposition $1.2, f^{j} p \in R_{+}-\left(R_{0} \cup R_{1}\right)$ for some $j \geq 1$. It follows that the complement of $\Omega_{+}$is contained in $W^{s}(\infty, \infty)$. If $w \in \Sigma_{+}$is not eventually alternating, then $R(w)$ is disjoint from $W^{s}(\infty, \infty)$. Since the set of all such $R(w)$ is a dense subset of $\Omega_{-}$and since $\Omega_{+}$is nowhere dense in $R_{0} \cup R_{1}$, the Lemma follows.

Theorem 9.3. $\overline{\mathbf{R}^{2}}-\mathcal{B}_{+}=\overline{\mathbf{R}^{2}} \cap \partial \mathcal{B}_{+}=\bigcup_{n \geq 0} f^{-n} \Omega_{+}$, and $\mathcal{B}_{+}$is dense in $\mathbf{R}^{2}$.

Proof. By Proposition 1.2, $f^{-1} \Omega_{+}-\Omega_{+} \subset R_{-}$. Thus $f^{-n-1} \Omega_{+}-f^{-n} \Omega_{+}$ tends uniformly to $(\infty, \infty)$ through $R_{-}$as $n \rightarrow \infty$. The Theorem then follows from the previous Lemma.

A point $p$ is said to be wandering if it has a neighborhood $U$ such that $U \cap f^{n}\left(U-I\left(f^{n}\right)\right)=\emptyset$ for all $n \neq 0$.

Proposition 9.4. The points of $\mathcal{B}_{+} \cup \mathcal{B}_{-}$are wandering.

Proof. If $p \in \mathcal{B}_{+}$, let $U$ be a neighborhood of $p$ on which $f^{n} \mid U$ converges uniformly to $(\infty, \infty)$. It follows that, shrinking $U$ if necessary, we have $f^{n} U \cap U=\emptyset$ for $n \geq 1$. Since $p \notin I\left(f^{n}\right)$ for $n \geq 0$, we also have that $f^{n} U \cap U=\emptyset$ for all $n \leq-1$.

Now suppose $p=(1,0)$. We have $\dot{f} p=\{y=a\} \subset R_{+} \cup R_{0}$ (see Figures 1 and 2). By Proposition 1.2, $\dot{f}^{n} p \subset R_{+} \cup R_{0} \cup R_{1}$ for all $n \geq 1$. It follows that if $U$ is a sufficiently small neighborhood of $p$, we have $f^{n}(U-\{p\}) \subset$ $R_{+} \cup R_{0} \cup R_{1}$ for all $n \geq 1$. In particular, we may choose $U$, so that $U \cap f^{n}(U-\{p\})=\emptyset$ for $n \geq 1$. On the other hand, since $(1,0) \in R_{-}$, we may choose $U$ small and apply Proposition 1.2 to have $f^{-n} U \cap U=\emptyset$ for $n \geq 1$.

Proposition 9.5. The lines at infinity $\{x=\infty\} \cup\{y=\infty\}$ are contained in the nonwandering set of $f$.

Proof. Since the nonwandering set is closed and invariant, it suffices to show that any point $p \in R_{0} \cap\{x=\infty\}-I_{-}$is nonwandering. Let $U$ be a neighborhood of $p$. Let us choose $N$ such that $f^{-N} p \in R_{0} \cap\{y=\infty\}$. By Theorem 6.5. $U$ intersects $f^{-m}\{x=1\}$ for sufficiently large $m$. Hence $f^{2 m} U \cap R_{0}$ contains a component connecting $\{x=1\}$ to $\{x=\infty\}$. Further, since this component is inside $R\left((10)^{2 m} \cdot\right)$ which is a small one-sided neighborhood of $R_{0} \cap\{y=\infty\}$, it must cross $f^{-N} U$.

Theorem 9.6. The nonwandering set is

$$
\{x=\infty\} \cup\{y=\infty\} \cup \Omega=\overline{\mathbf{R}^{2}}-\left(\mathcal{B}_{+} \cup \mathcal{B}_{-}\right) .
$$


Proof. The two sets above are equal by Theorem 9.3. By Proposition 9.4, this set contains the nonwandering set. The Theorem now follows from Proposition 9.5 and the fact that the periodic (saddle) points are dense in $\Omega \cap \mathbf{R}^{2}$.

\section{Stable MANifolds AND LAMinar CURRENTS}

This section is devoted to identifying the stable manifolds of points of $\Omega$. In order to do this, we define a pseudometric on $R_{0} \cup R_{1}$ which is uniformly contracted/expanded by $f$. Finally, we use the stable manifolds and transverse measures to construct stable and unstable currents, as was done in the Axiom A case by Ruelle and Sullivan [RS]. One motivation for doing this is to present all of the aspects of hyperbolicity present in $f$. Another motivation is that laminar currents have proved increasingly useful in understanding the dynamics of mappings (see, for instance $[\mathrm{Du}]$ ).

We say that $p \in \Omega_{+}$is accessible if there exists a continuous curve $\gamma$ : $[0,1] \rightarrow\left(R_{0} \cup R_{1} \cup R_{+}\right)$such that $\gamma([0,1)) \cap \Omega_{+}=\emptyset$, and $\gamma(1)=p$.

Theorem 10.1. The accessible points of $\Omega_{+}$are the points in the set

$$
\begin{gathered}
W^{s}(\infty, \infty) \cap \Omega_{+}=\Omega_{+} \cap \bigcup_{n \geq 0} f^{-n}\{x=1\} \\
=\left(R_{0} \cup R_{1}\right) \cap \bigcup_{n \geq 0} f^{-n}\{x=1\} .
\end{gathered}
$$

Proof. Suppose that $p \in \Omega_{+}$is accessible. Without loss of generality we may suppose that $p \in R_{0}$, and $\gamma \subset R_{0}$ is as in the definition. Let us assume, by way of contradiction, that $f^{n} p \notin\{x=1\}$ for all $n \geq 0$. Since $f^{n}(\gamma-\{p\}) \cap \Omega_{+}$, it follows that $f^{n}(\gamma) \cap\left(\{x=1\} \cap R_{0}\right)=\emptyset$. We may choose $n$ sufficiently large that $f^{n} p \in R_{0}$ and $f^{n}(\gamma) \cap R_{+} \neq \emptyset$, for otherwise $\gamma \subset \Omega_{+}$. Since $f^{n} \gamma \subset R_{0} \cup R_{1} \cup R_{+}$it follows that $f^{n} \gamma$ contains a point of $\{x=1\} \cap R_{0}$. This contradiction shows that the accessible points are contained in the displayed set.

Conversely, suppose that $f^{n} p \in\{x=1\} \cap R_{0}$. Then $f^{n+1} p \in R_{0} \cap\{x=$ $\infty\}$, and so $p \in \Omega_{+}$. Choose $\gamma$ to be a curve which has $f^{n} p$ as an endpoint and such that $\gamma-\left\{f^{n} p\right\} \subset \operatorname{int}\left(R_{+}\right)$. Then $f^{-n}(\gamma-\{p\}) \cap \Omega_{+}=\emptyset$, and $f^{-n} \gamma$ is an access to $p$.

Recall the homeomorphism $\mathcal{T}_{ \pm, j} \times I \cong \Omega_{ \pm, j}$ from the previous section. With this, we may define an ordering on $\mathcal{T}_{ \pm, j}$. For instance, if $w^{1}, w^{2} \in \mathcal{T}_{+, 0}$ (resp. $w^{1}, w^{2} \in \mathcal{T}_{+, 1}$ ) we say that $w^{1}<w^{2}$ if the s-arc $R\left(w^{1}\right) \cap \mathbf{R}^{2}$ lies to the left of (resp. below) $R\left(w^{2}\right) \cap \mathbf{R}^{2}$. It is evident that this defines total orders on $\mathcal{T}_{+, 0}$ and $\mathcal{T}_{+, 1}$. If $w^{1}, w^{2} \in \mathcal{T}_{+, j}$ and $w^{1} \leq w^{2}$, we define the interval $\left[w^{1}, w^{2}\right]=\left\{x \in \mathcal{T}_{+, j}: w^{1} \leq x \leq w^{2}\right\}$. By the product structure, we see that $\left[w^{1}, w^{2}\right]$ is a closed subset of $\mathcal{T}_{ \pm, j}$, and $\left(w^{1}, w^{2}\right)$ is open.

The map $f^{-1}$ induces maps $\sigma_{k}^{+}: \mathcal{T}_{+, 0} \rightarrow \mathcal{T}_{+, k}, k=0,1$ and $\sigma_{0}^{+}: \mathcal{T}_{+, 1} \rightarrow$ $\mathcal{T}_{+, 0}$ as follows. If $w=w_{0} \cdot w_{1} w_{2} \cdots \in \mathcal{T}_{+, 0}$, then for $k=0,1, f^{-1} R(w) \cap R_{k}$ is the s-arc $R\left(k \cdot w_{0} w_{1} \ldots\right)$. We write $\sigma_{k}^{+}(w)=k \cdot w_{0} w_{1} w_{2} \ldots$; thus $\sigma_{0}^{+}$and $\sigma_{1}^{+}$are the two right inverses of $\sigma_{+}$. We define $\sigma_{k}^{-}$in an analogous fashion.

Figure 8 shows how $f$ maps u-arcs in $R_{0}$. From this we can see that the restriction of $\sigma_{j}^{-}$to each $\mathcal{T}_{-, k}$ is either order-preserving or order-reversing. Thus we have: 

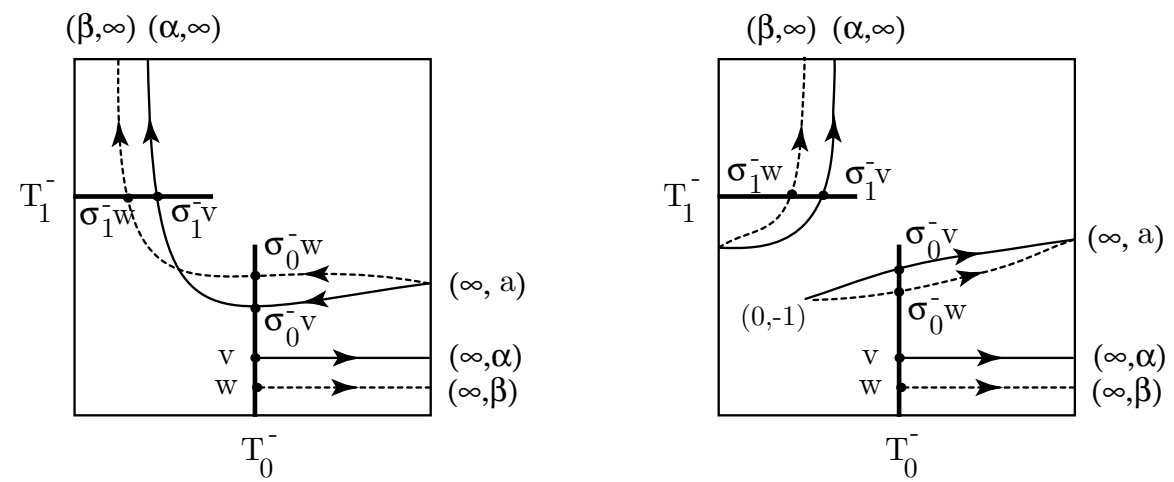

FiguRE 8 . Induced mappings $\sigma_{0}^{-}$and $\sigma_{1}^{-}$on the transversal $\mathcal{T}_{-}$.

Proposition 10.2. The mappings $\sigma_{k}^{ \pm}$take intervals to intervals.

The sets $\mathcal{T}_{ \pm, j}$ have the topologies of closed, perfect, totally disconnected subsets of $\mathbf{R}$. We may write the complement of $\mathcal{T}_{ \pm, j}$ in $\mathbf{R}$ as a union of open intervals $(a, b)$ : the points $w^{a}, w^{b} \in \mathcal{T}_{ \pm, j}$ corresponding to $a$ and $b$ are said to be accessible points of $\mathcal{T}_{ \pm, j}$. In terms of the order structure on $\mathcal{T}_{ \pm, j}$, these pairs of accessible points are characterized by the condition that the intervals $\left[w^{a}, w^{b}\right]$ contain only the endpoints $w^{a}$ and $w^{b}$.

Proposition 10.3. A point $w \in \mathcal{T}_{+, j}$ is accessible if and only if it lies in the stable manifold $W^{s}\left(\{\overline{01}, \overline{10}\}, \sigma_{+}, \Sigma_{+}\right)$.

Now we define a distance function dist $_{ \pm}^{\prime}$ on each of the sets $\mathcal{T}_{ \pm, j}$. For convenience of notation, let us work with $\dot{\mathcal{T}}_{+, 0}$. Let $\nu_{+}$denote the measure of maximal entropy for $\left(\sigma_{+}, \Sigma_{+}\right)$. For $w^{1}, w^{2} \in \mathcal{T}_{+, 0}$ we define

$$
\operatorname{dist}_{+}^{\prime}\left(w^{1}, w^{2}\right)=\nu_{+}\left(\left[w^{1}, w^{2}\right]\right) \geq 0
$$

If $w^{1}$ and $w^{2}$ are not both accessible, then $\left[w^{1}, w^{2}\right]$ contains an open subset of $\Sigma_{+}$, so $\operatorname{dist}^{\prime}\left(w^{1}, w^{2}\right)=\nu_{+}\left(\left[w^{1}, w^{2}\right]\right)>0$. An immediate consequence of the balanced property of $\nu_{+}$is:

Proposition 10.4. For $v, w \in \mathcal{T}_{ \pm, k}$, $\operatorname{dist}_{ \pm}^{\prime}\left(\sigma_{j}^{ \pm} v, \sigma_{j}^{ \pm} w\right)=\phi^{-1} \operatorname{dist}_{ \pm}^{\prime}(v, w)$.

Now we may extend dist $_{ \pm}^{\prime}$ to $R_{j}$ by the following requirement: if $U$ is a connected component of $R_{j}-\Omega_{+}$, then we set $\operatorname{dist}_{+}^{\prime}(p, q)=0$ for all $p, q \in U$. Thus $\operatorname{dist}^{\prime}:=\max \left(\right.$ dist $_{+}^{\prime}$, dist $\left._{-}^{\prime}\right)$ is a pseudodistance on $R_{j}$ for $j=0,1$. Since $R_{0} \cap R_{1}=\{(\infty, \infty)\}$, we extend dist' to $R_{0} \cup R_{1}$ by setting $\operatorname{dist}^{\prime}(p, q)=\operatorname{dist}^{\prime}(p,(\infty, \infty))+\operatorname{dist}^{\prime}(q,(\infty, \infty))$ whenever $p \in R_{0}$ and $q \in R_{1}$.

Recall from Proposition 1.2 that if $p \notin W^{s}(\infty, \infty) \cup I_{+}$, then $f^{n} p \in$ $R_{0} \cup R_{1}$ for $n$ sufficiently large. This motivates the following definition. For $p \in \overline{\mathbf{R}^{2}}-\left(W^{s}(\infty, \infty) \cup I_{+}\right)$, we set

$$
W^{s}(p):=\left\{q \in \overline{\mathbf{R}^{2}}-\left(W^{s}(\infty, \infty) \cup I_{+}\right): \lim _{n \rightarrow+\infty} \operatorname{dist}^{\prime}\left(f^{n} p, f^{n} q\right)=0\right\} .
$$

Theorem 10.5. Let $p \in \Omega-W^{s}(\infty, \infty)$ be given, and let $w \in \Sigma$ be such that $p=R(w)$. Then $\dot{W}^{s}(p):=\bigcup_{n \geq 0} f^{-n} R\left(\left(\sigma^{n} w\right)^{+}\right)$is equal to $W^{s}(p) \cup I_{+}$, and $W^{s}(p)=\dot{W}^{s}(p)-I_{+} \subset \mathbf{R}^{2}$. 
Proof. On $R\left(w^{+}\right)$, dist ${ }^{\prime}$ is equal to dist' and there decreases by factors of $\phi$ under forward iteration, so $R\left(w^{+}\right) \subset W^{s}(p)$. Thus $\dot{W}^{s}(p)-I_{+} \subset W^{s}(p)$.

Conversely, let us suppose that $q \in W^{s}(p)-\left(W^{s}(\infty, \infty) \cup I_{+}\right)$. Let $U$ be a neighborhood of $(\infty, \infty)$ such that $f$ is a diffeomorphism from $U \cap R_{0}$ (resp. $\left.U \cap R_{1}\right)$ to $f U \cap R_{1}$ (resp. $f U \cap R_{0}$ ). Choose $\epsilon>0$ such that an $\epsilon$ neighborhood of $(\infty, \infty)$ with respect to dist ${ }^{\prime}$ is contained in $U$. For $N$ sufficiently large, we may assume that $\operatorname{dist}^{\prime}\left(f^{n} p, f^{n} q\right)<\epsilon / 2$ for all $n \geq N$. By Proposition 1.2, $f^{n} p, f^{n} q \in R_{0} \cup R_{1}$ for $n$ sufficiently large. We claim that $f^{n} p$ and $f^{n} q$ must be contained in the same rectangle for $n \geq N+1$. Thus it will follow that $f^{N+1} q \in R\left(\sigma^{N+1} w\right)$, which means that $q \in \dot{W}^{s}(p)$. To see why the claim is true, observe that if $f^{n} p \in R_{0} \cap \Omega$ and $f^{n} q \in R_{1} \cap \Omega$ are points in different rectangles, then since $f^{n} p, f^{n} q \in U$ for all $n \geq N$, we have $f^{n+1} p \in R_{1}$ and $f^{n+1} q \in R_{0}$. Thus $f^{n} p$ and $f^{n} q$ are in opposite $R_{j}$ 's for $n>N$, which means that $p$ and $q$ would have to corresponding to eventually alternating words, which is not possible since $p \notin W^{s}(\infty, \infty)$.

Corollary 10.6. If $p \in \Omega-W^{s}(\infty, \infty)$, then $W^{s}(p) \cap\left(R_{0} \cup R_{1}\right) \subset \Omega_{+}$. If $p \in \Omega-W^{s}(\infty, \infty)$ and $q \in \Omega-W^{u}(\infty, \infty)$, then $W^{s}(p) \cap W^{u}(q) \subset \Omega$.

Let us define

$$
\mathcal{W}^{s}=\mathbf{R}^{2} \cap \bigcup_{n \geq 0} f^{-n} \Omega_{+}-I_{+}, \quad \mathcal{W}^{u}=\mathbf{R}^{2} \cap \bigcup_{n \geq 0} f^{n} \Omega_{-}-I_{-} .
$$

Theorem 10.7. $\mathcal{W}^{s}$ is a lamination of $\mathbf{R}^{2}-I_{+}$; the leaves of $\mathcal{W}^{s}$ are the connected components of $W^{s}(p) \cap \mathbf{R}^{2}$ for $p \in \mathcal{D} \cap \Omega$ and of $\mathbf{R}^{2} \cap f^{-n}\{x=$ $1\}-\mathcal{I}_{+}$for $n \geq 0$. Similarly, $\mathcal{W}^{u}$ is a lamination of $\mathbf{R}^{2}-I_{-}$; the leaves of $\mathcal{W}^{u}$ are the connected components of $W^{u}(p)$ for $p \in \mathcal{D} \cap \Omega$ and of $\mathbf{R}^{2} \cap f^{n}\{y=$ $-1\}-I_{-}$for $n \geq 0$.

Proof. We have seen that $\Omega_{+} \cap R_{j} \cap \mathbf{R}^{2}$ is homeomorphic to $\mathcal{T}_{+, j} \times I$, and thus $\Omega_{+} \cap \mathbf{R}^{2}$ is a lamination of $\left(R_{0} \cup R_{1}\right) \cap \mathbf{R}^{2}$. Now $f^{-n-1} \Omega_{+} \supset f^{-n} \Omega_{+}$, and $f^{-n-1} \Omega_{+}-f^{-n} \Omega_{+} \subset \operatorname{int} R_{-}$. Thus $f^{-1} \Omega_{+} \cap \mathbf{R}^{2}$ is a lamination except at the image of the critical locus of $f^{-1}$, which is $I(f)$. The backward orbit of $I(f)$ is $I_{+}$. Thus this theorem follows from Theorem 10.5.

The left side of Figure 9, which shows $f^{-10}(\{x=1.5\})$, gives an approximation of $\mathcal{W}^{s}$. Note that we have replaced the usual Euclidean coordinates $(x, y)$ on $\mathbf{R}^{2}$ with new coordinates $\left(x^{\prime}, y^{\prime}\right)=(\arctan x, \arctan y)$ in order to better show the behavior near infinity. Note also that the image curve intersects itself precisely at points in $I\left(f^{10}\right)$, and these are in the backward orbit of $\{(-a, \infty),(1,0)\}$. Both $\mathcal{W}^{s}$ and $\mathcal{W}^{u}$ are given on the right hand side of Figure 9. The intersection of the two curves approximates $\Omega$.

Now we introduce some standard terminology from the theory of currents. A good reference for this is Morgan [M]. For an oriented arc $\gamma$ of finite length, we define the current of integration $[\gamma]$, which is an object that acts on a 1-form $\xi$ as follows

$$
\langle[\gamma], \xi\rangle:=\int_{\gamma} \xi
$$

Since $\gamma$ has finite length, $\langle[\gamma], \xi\rangle$ is dominated by the length of $\gamma$ and $\sup _{\gamma}|\xi|$. In this case, $[\gamma]$ is said to be represented by integration. Currents which are represented by integration may be treated as vector-valued measures in the sense that they may be written the form $\vec{t} \cdot \lambda$, where $\vec{t}$ is a Borel measurable 


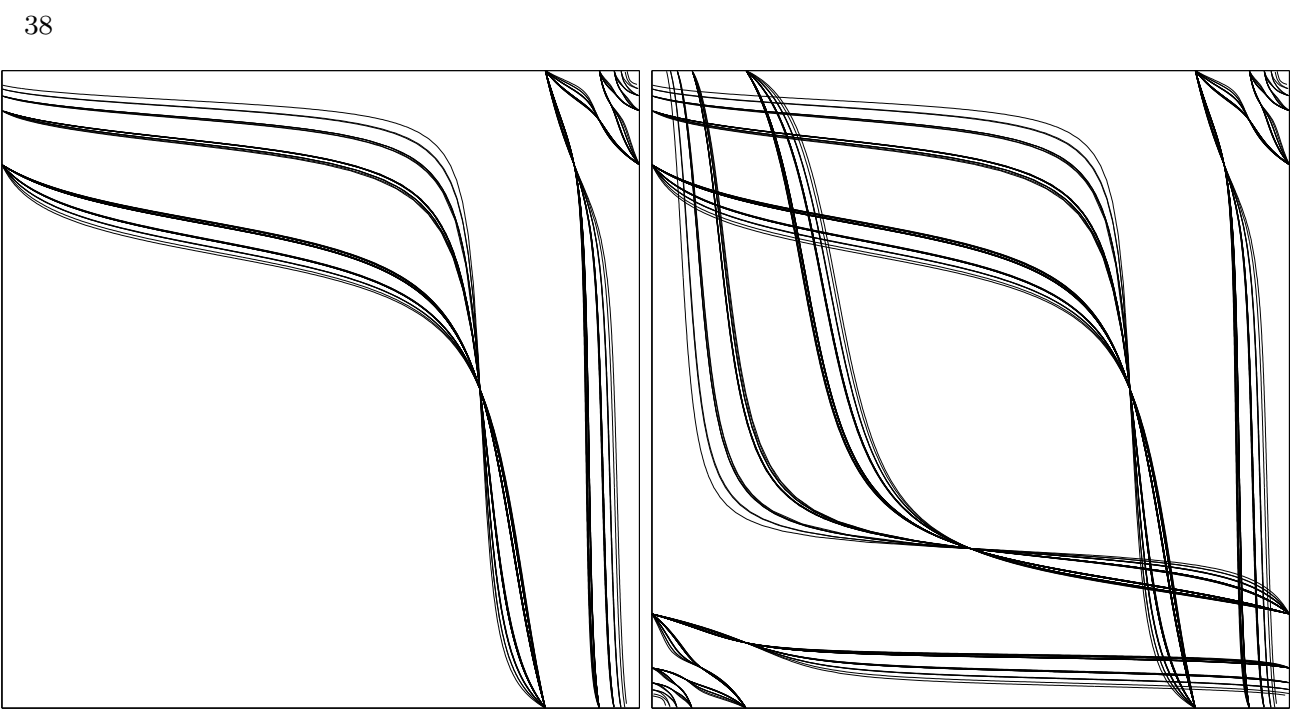

Figure 9. Stable lamination (left), both laminations (right), $a=-2$.
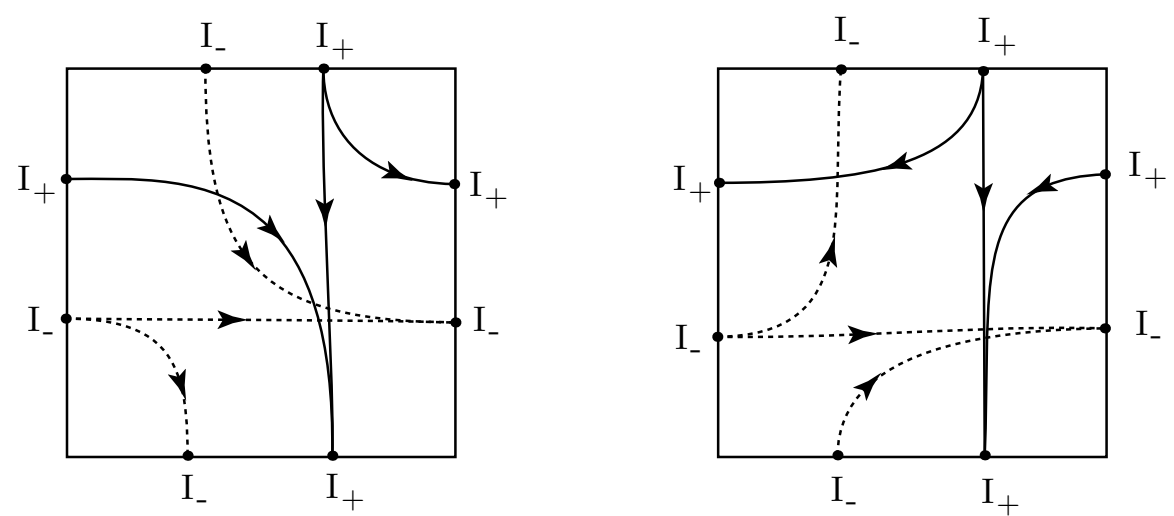

FiguRE 10. Orientations of stable and unstable currents.

1-vector, and $\lambda$ is a Borel measure. Further, it follows that for any smooth function $h,\langle[\gamma], d h\rangle=h(Q)-h(P)$, where $P$ and $Q$ are the endpoints of $\gamma$. In other words, the boundary of the current $[\gamma]$ is a difference of point masses: $\partial[\gamma]=\delta_{Q}-\delta_{P}$.

It is an elementary consequence of our slope bounds that:

Proposition 10.8. The length of $R(w)$ as a curve in $\overline{\mathbf{R}^{2}}$ is uniformly bounded for all $w \in \Sigma_{+}$.

For $w \in \mathcal{T}_{j}^{+}$, we assign to the arc $R(w) \cap \mathbf{R}^{2}$ the orientation for which it points down and to the right. A schematic diagram of this orientation is given for $a<-1$ on the left hand side of Figure 10. Note that this diagram in fact shows how to orient the whole stable lamination $\mathcal{W}^{s}$. The orientation for $\mathcal{W}^{u}$, obtained by mapping under $(x, y) \mapsto(-y,-x)$, is also given in Figure 10.

The current $\left[R(w) \cap \mathbf{R}^{2}\right]$ depends continuously on $w$, and we define

$$
\mu_{j}^{+}=\int_{w \in \mathcal{T}_{+, j}}\left[R(w) \cap \mathbf{R}^{2}\right] \nu^{+}(w) .
$$


The action of $\mu_{j}^{+}$on a 1 -form $\xi$ is given by:

$$
\left\langle\mu_{j}^{+}, \xi\right\rangle:=\int_{w \in \mathcal{T}_{+, j}}\left\langle\left[R(w) \cap \mathbf{R}^{2}\right], \xi\right\rangle \nu_{+}(w) .
$$

In other words, this is a direct integral of currents of integration: for each $w \in \mathcal{T}_{+, j}$, we apply the current of integration $[R(w)]$ to the test form $\xi$ on $\mathbf{R}^{2}$, and we then integrate the result with respect to $\nu_{+}$over the set $w \in \mathcal{T}_{+, j}$.

Proposition 10.9. $\mu_{j}^{+}$is a current on $\mathbf{R}^{2}$ whose support is contained in $\Omega^{+} \cap R_{j}$. The support of its boundary, $\partial \mu_{j}^{+}$is contained in $\partial^{u} R_{j}$.

Now let us see how $\mu_{0}^{+}$transforms under $f$. The pull-back of the direct integral is the direct integral of the pull-back. Therefore we obtain

$$
f^{*} \mu_{0}^{+}=\int_{w \in \mathcal{T}_{0}^{+}} f^{*}\left[R(w) \cap \mathbf{R}^{2}\right] \nu_{+}(w)=\int_{w \in \mathcal{T}_{0}^{+}}\left[f^{-1}\left(R(w) \cap \mathbf{R}^{2}\right)\right] \nu_{+}(w) .
$$

We compare $f^{*} \mu_{j}^{+}$with $\mu_{j}^{+}$. Since $\mu_{j}^{+}$is represented by integration, we may multiply it by $\mathbf{1}_{R_{0} \cup R_{1}}$, the characteristic function of the set $R_{0} \cup R_{1}$. We know that for each $w, f^{-1}\left(R(w) \cap \mathbf{R}^{2}\right)$ crosses both $R_{0}$ and $R_{1}$ in s-arcs, as is shown in Figure 8. Figure 8 shows that the orientations of $f^{-1}\left(R(w) \cap \mathbf{R}^{2}\right)$ are opposite from the orientations in Figure 10. By the definition of $\sigma_{k}^{+}$, we see that

$$
\mathbf{1}_{R_{0} \cup R_{1}}\left[f^{-1}\left(R(w) \cap \mathbf{R}^{2}\right)\right]=-\left[R\left(\sigma_{0}^{+} w\right)\right]-\left[R\left(\sigma_{1}^{+} w\right)\right] .
$$

If we take the integral with respect to $\nu_{+}$, we have

$$
\mathbf{1}_{R_{0} \cup R_{1}} f^{*} \mu_{0}^{+}=-\int_{w \in \mathcal{T}_{+, 0}} \nu_{+}(w)\left(\left[R\left(\sigma_{0}^{+} w\right)\right]+\left[R\left(\sigma_{1}^{+} w\right)\right]\right) .
$$

By the balanced property of $\nu_{+}$, this is

$$
=-\phi \int_{v_{0} \in \sigma_{0}^{+}\left(\mathcal{T}_{+, 0}\right) \subset \mathcal{T}_{+, 0}} \nu_{+}\left(v_{0}\right)\left[R\left(v_{0}\right)\right]-\phi \int_{v_{1} \in \sigma_{1}^{+}\left(\mathcal{T}_{+, 0}\right)=\mathcal{T}_{+, 1}} \nu_{+}\left(v_{1}\right)\left[R\left(v_{1}\right)\right] .
$$

Applying the same reasoning to $\mathbf{1}_{R_{0} \cup R_{1}} f^{*} \mu_{1}^{+}$, and adding, we obtain:

Proposition 10.10.

$$
\mathbf{1}_{R_{0} \cup R_{1}} f^{*}\left(\mu_{0}^{+}+\mu_{1}^{+}\right)=-\phi\left(\mu_{0}^{+}+\mu_{1}^{+}\right) .
$$

Now we consider the sequence of currents $(-\phi)^{-n} f^{* n}\left(\mu_{0}^{+}+\mu_{1}^{+}\right)$, which coincide with $\mu_{0}^{+}+\mu_{1}^{+}$on the interior of $R_{0} \cup R_{1}$. We may now argue as in the proof of Theorem 10.8 to obtain:

Theorem 10.11. The current $\mu_{0}^{+}+\mu_{1}^{+}$extends to a closed current $\mu_{\mathbf{R}}^{+}$on $\mathbf{R}^{2}-I_{+}$, which satisfies $f^{*} \mu_{\mathbf{R}}^{+}=-\phi \cdot \mu_{\mathbf{R}}^{+}$.

In a similar way, we may define currents

$$
\mu_{j}^{-}=\int_{w \in \mathcal{T}_{-, j}}\left[R(w) \cap \mathbf{R}^{2}\right] \nu^{-}(w),
$$

and we may extend $\mu_{0}^{-}+\mu_{1}^{-}$to a closed current $\mu_{\mathbf{R}}^{-}$on $\mathbf{R}^{2}-I_{-}$, which satisfies $f^{*} \mu_{\mathbf{R}}^{-}=-\phi \mu_{\mathbf{R}}^{-}$.

Let $\gamma_{1}$ and $\gamma_{2}$ be oriented arcs of finite length, and let $\left[\gamma_{1}\right]$ and $\left[\gamma_{2}\right]$ be their currents of integration. We define the wedge (intersection) product of these currents to be $\left[\gamma_{1}\right] \wedge\left[\gamma_{2}\right]=0$ if the supports of $\left[\gamma_{1}\right]$ and $\left[\gamma_{2}\right]$ are 


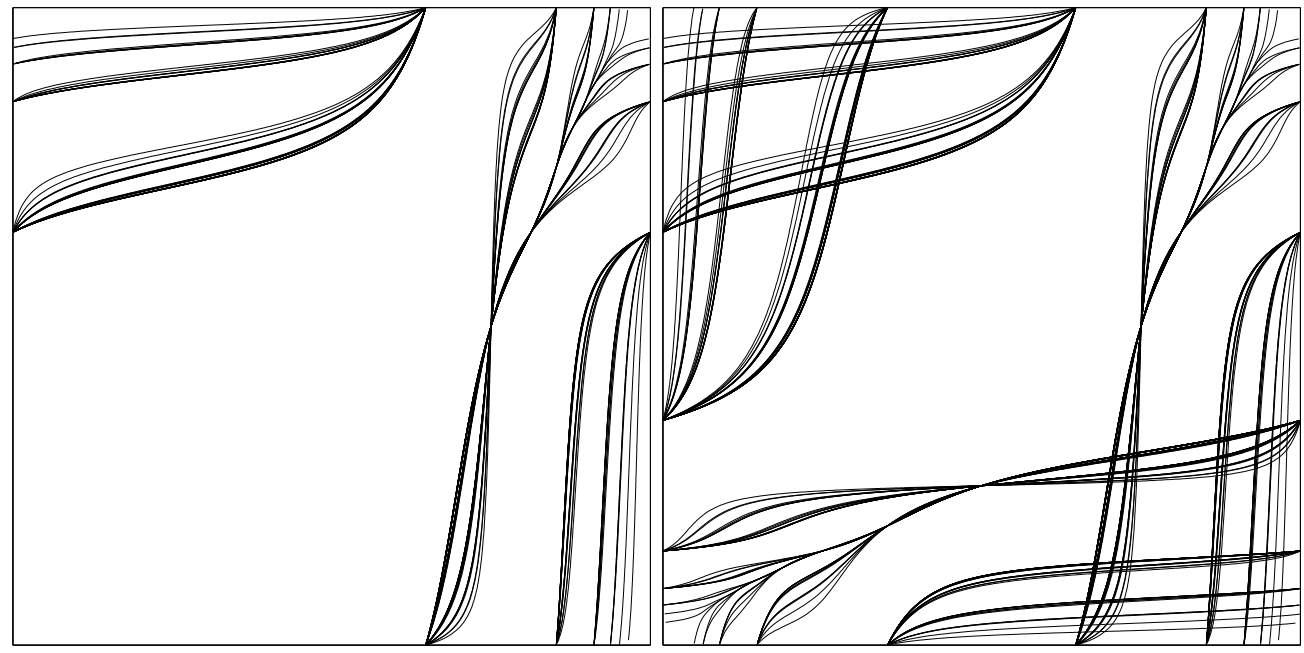

Figure 11. Stable lamination (left), both laminations (right), $a=-.5$

disjoint. If $\gamma_{1} \cap \gamma_{2}$ consists of a single point $p$, where the curves intersect transversally, we define $\left[\gamma_{1}\right] \wedge\left[\gamma_{2}\right]= \pm \delta_{p}$ to be the (signed) point mass at $p$, with the choice of sign taken to be positive if wedge products of the tangents at $p$ agrees with the orientation of $\mathbf{R}^{2}$ at $p$. Let us orient $R_{j}$ such that for $w \in \Sigma^{\prime}$ we have $\left[R\left(w^{+}\right)\right] \wedge\left[R\left(w^{-}\right)\right]=\delta_{R(w)}$.

The wedge product of the direct integrals is the direct integral of the wedge products, so we obtain

$$
\begin{aligned}
\mu_{\mathbf{R}}^{+} \wedge \mu_{\mathbf{R}}^{-} & =\int_{w \in \Sigma_{+}} \int_{\tilde{w} \in \Sigma_{-}}[R(w)] \wedge[R(\tilde{w})] \nu^{+}(w) \otimes \nu^{-}(\tilde{w}) \\
& =\int_{\tilde{w}_{0}=w_{0}} \delta_{R(w) \cap R(\tilde{w})} \nu^{+}(w) \otimes \nu^{-}(\tilde{w})
\end{aligned}
$$

From $\S 2$ we recall the product structure map

$$
\mathcal{T}_{+, j} \times \mathcal{T}_{-, j} \ni\left(w^{+}, w^{-}\right) \mapsto \tilde{w} \in \Sigma^{\prime} \cap \pi^{-1}(j),
$$

defined by $\tilde{w}^{ \pm}=w^{ \pm}$. With this map, we have the relation $\nu^{+} \otimes \nu^{-} \cong \nu$. In $\S 8$ we discussed the product structure mapping

$$
R: \mathcal{T}_{j,+} \times \mathcal{T}_{j,-} \ni\left(w^{+}, w^{-}\right) \mapsto R(\tilde{w}) \in \Omega \cap R_{j} \cap \mathbf{R}^{2} .
$$

This map was used to define $\mu$ as the pushforward of $\nu=\nu^{+} \otimes \nu^{-}$. Since these two product structures coincide, we have:

Theorem 10.12. $\mu=\mu_{\mathbf{R}}^{+} \wedge \mu_{\mathbf{R}}^{-}$.

\section{Parameter Values $-1<a<0$}

The main results that we have proved so far hold for all parameters $a<0$ save the exceptional value $a=-1$. However, in our proofs we have been assuming that $a<-1$. The details in the case $-1<a<0$ are similar enough that most of them are not worth repeating. We will use this section to point out those few places in which the differences are significant.

The first and most basic change is that the definition of the filtration $R_{0}, R_{1}, R_{ \pm}$must be changed so that the lines $\{x=-a\}$ and $\{y=a\}$ take the place of the lines $\{x=1\}$ and $\{y=-1\}$. In other words, $R_{1}$ is unchanged, but $R_{0}=\{-a \leq x\} \cap\{y \leq a\}$. Proposition 1.2 then holds with a slight 


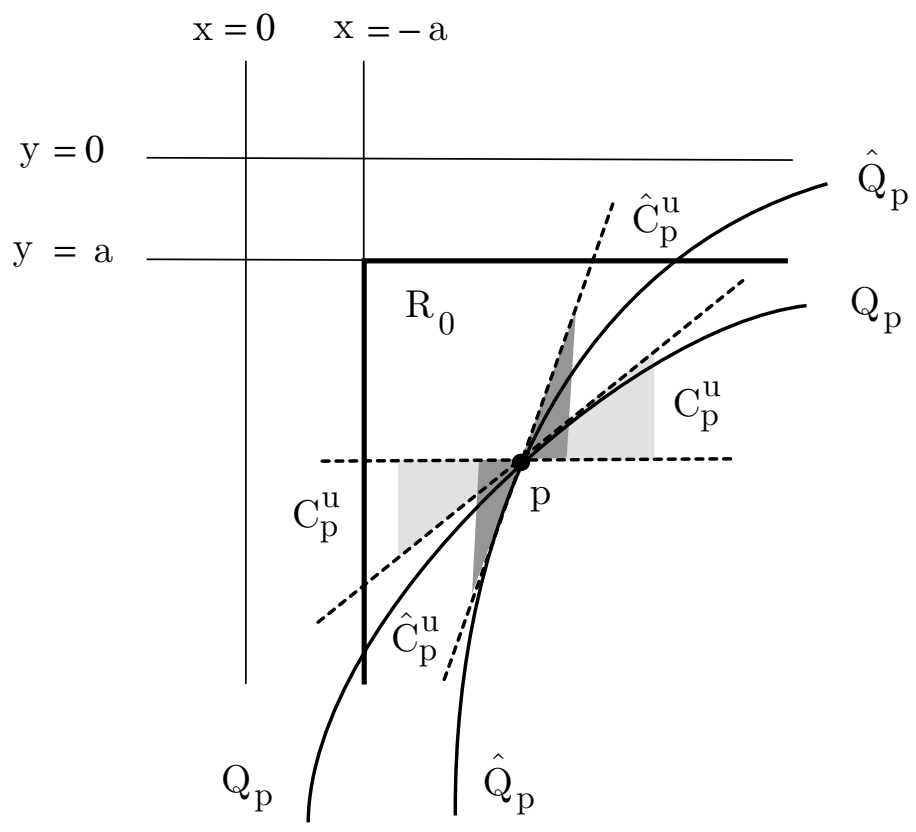

Figure 12. Cone Fields for $-1<a<0$

alteration to the first two items. For example, the first item should read: if $\left(x_{0}, y_{0}\right) \in R^{+}$, then

$$
\max \left\{x_{1}+a, y_{1}\right\} \leq \max \left\{x_{0}+a, y_{0}\right\}+a .
$$

The definitions of s-arcs and $\mathrm{u}$-arcs do not change. Nor does Proposition 2.2. We define the stable/unstable cones as follows. For $p \in R_{0}$, we let $Q_{p}$ denote the branch of the hyperbola (quadric) passing through $p$ and having $\{x=0\}$ and $\{y=a\}$ as asymptotes. We let $\hat{Q}_{p}$ denote the hyperbola passing through $p$ and having $\{x=a\}$ and $\{y=0\}$ as asymptotes. For $p \in R_{0}$, we define $\mathcal{C}_{p}^{u}$ (respectively, $\hat{\mathcal{C}}_{p}^{u}$ ) to be the cone of vectors swept out by starting at the horizontal $H_{p}$ and moving counter-clockwise to the tangent to $Q_{p}$ (respectively $\hat{Q}_{p}$ ) at $p$. This is shown in Figure 12. The following is proved along the same general lines as Theorems 5.1 and 5.2.

Theorem 11.1. If $p, f p, f^{2} p \in\left(R_{0} \cup R_{1}\right) \cap \mathbf{R}^{2}$, then $D f_{p} \hat{\mathcal{C}}_{p}^{u} \subset \mathcal{C}_{f p}^{u}$. Further, $D f_{p}^{2}$ maps $\mathcal{C}_{p}^{u}$ strictly inside $\mathcal{C}_{f^{2} p}^{u}$.

From this theorem, we derive the slope bounds analogous to those of Theorem 5.3. We change the definitions of uniform s-arcs and u-arcs to fit the new slope bounds. Despite the change in particulars, the role of the stable and unstable cones is exactly as before. For instance, the angle of intersection between a uniform $s$-arc and a uniform $u$-arc is bounded away from zero uniformly on any compact subset of $\mathbf{R}^{2}$.

All of the assertions in Sections 6-10 remain true without change for $-1<a<0$. However, we note that in Section 7 some of the proofs become a little easier. The reason for this can be seen in the approximation of $f^{2}$ presented in Proposition 1.1. For $x$ and $y$ large and $a$ between 0 and -1 , we have that $x$ decreases and that $y$ increases with every iterate of $f^{2}$. This was not quite true in the case $a<-1$. 
The most important difference between the cases $a<-1$ and $-1<a<0$ concerns orientation. We orient uniform $u$-arcs and $s$-arcs so that their tangent vectors point up and to the right; a schematic illustration is given on the right hand side of Figure 10. In this case, $f$ preserves, rather than reverses, their orientations. Thus we have the transformation law

$$
\left.f^{*} \mu_{\mathbf{R}}^{+}\right|_{R_{0} \cup R_{1}}=\phi \mu_{\mathbf{R}}^{+}
$$

for the current $\mu_{\mathbf{R}}^{+}$supported on $\Omega^{+}$.

\section{The PuRely COMPlex POINT OF VIEW}

We have given a detailed description of the dynamics of the specific family $\left\{f_{a}: a<0, a \neq-1\right\}$ of birational maps of $\mathbf{R}^{2}$. There is also a general theory which applies to birational mappings of $\mathbf{C}^{2}$. In this Section we state a result of $[\mathrm{DF}]$ on the existence of invariant currents and two results from [BD] on the existence of invariant measures. Then we show how the results obtained in the preceding sections fit into the framework of the complex theory.

From $\S 3$ recall the basis $\left\{\gamma_{1}^{*}, \gamma_{2}^{*}\right\}$ of $H^{2}\left(\mathbf{P}^{1} \times \mathbf{P}^{1}\right)$ and the action of $f^{*}$ on $H^{2}\left(\mathbf{P}^{1} \times \mathbf{P}^{1}\right)$. The $\phi$ eigenspace of $f^{*}$ is generated by the cohomology class $\theta^{+}:=c\left(\gamma_{1}^{*}+\phi \gamma_{2}^{*}\right)$ for any $c \neq 0$. The generator $\theta^{-}$for the $\phi$ eigenspace of $f_{*}=\left(f^{-1}\right)^{*}$ is given by $c\left(\phi \gamma_{1}^{*}+\gamma_{2}^{*}\right)$. We choose $c=\left(1+\phi^{2}\right)^{-1 / 2}>0$ so that $\theta^{+} \cdot \theta^{-}=1$. Because $f^{*}$ and $f_{*}$ are adjoint with respect to the intersection product, standard linear algebra, applied to the 2-dimensional space $H^{2}$, gives

$$
\lim _{n \rightarrow \infty} \frac{f^{n *} \theta}{\phi^{n}}=\left(\theta \cdot \theta^{-}\right) \theta^{+}
$$

for any class $\theta \in H^{2}\left(\mathbf{P}^{1} \times \mathbf{P}^{1}\right)$.

A current $T$ of bidimension $(1,1)$ is said to be positive if $\left\langle T, \frac{1}{i} \alpha \wedge \bar{\alpha}\right\rangle \geq 0$ for any $(1,0)$ form $\alpha$ on $\mathbf{P}^{1} \times \mathbf{P}^{1} . \quad f$ induces a well-defined action, also denoted by $f^{*}$, on the space of positive, closed currents (see [Gu1] or [S]). In fact, if $\omega$ is a current representing a cohomology class $\theta$, then $f^{*} \omega$ is a current representing the cohomology class $f^{*} \theta$. If $\omega$ is smooth, then $f^{n *} \omega$ is smooth except at the finite set $I\left(f^{n}\right)$. The action of $f^{*}$ on positive, closed currents closely follows the action of $f^{*}$ on cohomology:

Theorem 12.1. There exists a unique positive, closed $(1,1)$ current $\mu^{+}$on $\mathbf{P}^{1} \times \mathbf{P}^{1}$ with the following properties.

- $\mu^{+}$represents $\theta^{+}$;

- $f^{*} \mu^{+}=\phi \mu^{+}$;

- For every cohomology class $\theta \in H^{2}(X)$ and every smooth form $\omega$ representing $\theta$,

$$
\lim _{n \rightarrow \infty} \frac{f^{n *} \omega}{\phi^{n}}=\left(\theta \cdot \theta^{-}\right) \mu^{+}
$$

Applying the previous theorem to $f^{-1}$, we obtain an invariant current $\mu^{-}$in the cohomology class of $\theta^{-}$. Thus, $\mu^{-}=\phi^{-1}\left(f^{-1}\right)^{*} \mu^{-}=\phi^{-1} f_{*} \mu^{-}$. Because

$$
\overline{I_{+}} \cap \overline{I_{-}}=\{(\infty, \infty)\}
$$

contains only one point, we also have

Theorem 12.2. The wedge product $\mu=\mu^{+} \wedge \mu^{-}$is well-defined and equal to an $f$-invariant ergodic probability measure on $\mathbf{P}^{1} \times \mathbf{P}^{1}$. 
It is useful to know that the limit in the third item of the first theorem can be freely interchanged with the wedge product defining $\mu$. Thus the measure $\mu$ arises from simultaneously pushing forward and pulling back arbitrary smooth currents.

Theorem 12.3. Let $\theta, \tilde{\theta} \in H^{2}\left(\mathbf{P}^{1} \times \mathbf{P}^{1}\right)$ be classes represented by smooth currents $\omega, \tilde{\omega}$. Then

$$
c \mu=\lim _{n, m \rightarrow \infty} \frac{f^{n *} \omega \wedge f_{*}^{m} \tilde{\omega}}{\phi^{n+m}},
$$

where $c=\left(\theta \cdot \theta^{-}\right)\left(\tilde{\theta} \cdot \theta^{+}\right)$.

Let us consider the cohomology class $\gamma_{2}^{*}$ generated by any vertical $\{x=t\}$. If $\chi \in C_{0}^{\infty}(\mathbf{R}), \int \chi=c$, then

$$
\omega:=\int\left[V_{t}\right] \chi(t) d t
$$

is a current which represents $c \gamma_{2}^{*}$. Similarly, if $\tilde{\chi} \in C_{0}^{\infty}(\mathbf{R}), \int \tilde{\chi}=\tilde{c}$, and if $H_{s}=\{y=x\}$, then

$$
\tilde{\omega}:=\int\left[H_{s}\right] \tilde{\chi}(s) d s
$$

is a current representing the cohomology class $\tilde{c} \gamma_{1}^{*}$ of a horizontal line. We choose $c, \tilde{c}>0$ so that $\left(\theta^{-} \cdot \omega\right)\left(\theta^{+} \cdot \tilde{\omega}\right)=1$. While $\omega$ and $\tilde{\omega}$ are not smooth on $\mathbf{P}^{1} \times \mathbf{P}^{1}$, they have continuous potentials, and Theorem 12.3 can be adapted to cover this case. It follows, then, that $\phi^{-(n+m)} f^{* n} \omega \wedge f_{*}^{m} \tilde{\omega}$ converges to the measure $\mu$ of Theorem 12.2 as $n, m \rightarrow \infty$.

Theorem 12.4. Suppose that $a<0, a \neq-1$. Then for any $s \leq 0, t \geq 1$, we have

$$
\mu=\frac{1+\phi^{2}}{\phi^{2}} \lim _{n, m \rightarrow \infty} \phi^{-n-m} \sum_{a \in f^{-n} V_{s} \cap f^{m} H_{t}} \delta_{a},
$$

where the convergence is in the weak sense of measures.

Proof. With the notation as above, let us suppose that $\chi$ is supported in $\{1 \leq x<\infty\}$, and thus for each $t$ in the integral defining $\omega, V_{t}$ intersects $R_{0} \cup R_{1}$ in a uniform s-arc. Similarly, if $\tilde{\chi}$ is supported in $\{-\infty<y \leq-1\}$, then for each $s$ in the integral defining $\tilde{\omega}, H_{s}$ intersects $R_{0} \cup R_{1}$ in a uniform u-arc. Now let us consider fixed $n, m>0$, and let $w$ be a word of extent $[-n, m]$. By $\S 4$, it follows that $f^{-n} V_{t} \cap f^{m} H_{s}$ intersects $R(w)$ in exactly one point, and the union over all $w$ gives $f^{-n} V_{t} \cap f^{m} H_{s}$. Let us denote this point by $p(s, t, w)$. It follows that the restriction of $f^{m *} \omega \wedge f_{*}^{n} \tilde{\omega}$ to $R(w)$ is given by the integral

$$
I_{w}:=\int_{s \in \mathbf{R}} \int_{t \in \mathbf{R}} \delta_{p(s, t, w)} \chi(s) \tilde{\chi}(t) d s d t
$$

Next let us note that as a consequence of Theorem 8.1, we have the following: if $K$ is a compact subset of $\mathbf{R}^{2}$, then

$$
\lim _{m, n \rightarrow \infty} \max \{\operatorname{diam}(R(w) \cap K)\}=0,
$$

where the maximum is taken over all words $w$ of extent $[-n, m]$. By Theorem 12.3, we know that the sum of the integrals $\sum_{w} I_{w}$ converges to $\frac{\phi^{2}}{1+\phi^{2}} \mu$ as $n, m \rightarrow \infty$. Since the diameters of the $R(w)$ shrink to zero uniformly on any compact set $K$, it follows that the difference between $\sum_{w} I_{w}$ and 
$\phi^{-n-m} \sum_{a \in f^{-n} V_{s} \cap f^{m} H_{t}} \delta_{a}$ tends to 0 as $n, m \rightarrow \infty$. This proves the Theorem.

As was seen in the proof of the preceding theorem, each measure $I_{w}$ has the same mass, and so $f^{n *} \omega \wedge f_{*}^{m} \tilde{\omega}$ puts the same mass on each the rectangle $R(w)$. On the other hand, a well known property of the measure $\nu$ is that it can be obtained by equidistributing mass over the "cylinder sets"

$$
C(w)=\left\{s \in \Sigma: s_{j}=w_{j} \text { for }-n \leq j \leq m\right\}
$$

and letting $n, m \rightarrow \infty$. Now let us recall that the measure $\mu$ in $\S 8$ was obtained as the image of $\nu$ under the map $R$. Since $R$ takes a cylinder $C(w)$ to a rectangle $R(w)$, we have the following:

Theorem 12.5. Suppose that $a<0, a \neq-1$. Then the measure $\mu$ defined in this section is the same as the measure $\mu$ defined in $\$ 8$.

\section{References}

[Ab1] N. Abarenkova, J.-Ch. Anglès d'Auriac, S. Boukraa, S. Hassani and J.-M. Maillard, Rational dynamical zeta functions for birational transformations, Physica A 264 (1999) 264-293.

[Ab2] N. Abarenkova, J.-Ch. Anglès d'Auriac, S. Boukraa, S. Hassani and J.-M. Maillard, Topological entropy and complexity for discrete dynamical systems, Phys. Lett. A 262 (1999) 44-49.

[Ab3] N. Abarenkova, J.-Ch. Anglès d'Auriac, S. Boukraa, S. Hassani and J.-M. Maillard, Growth complexity spectrum of some discrete dynamical systems, Physica D 130 (1999) 27-42.

[Ab4] N. Abarenkova, J.-Ch. Anglès d'Auriac, S. Boukraa and J.-M. Maillard, Real Arnold complexity versus real topological entropy for birational transformations, J. Phys. A. 33 (2000), 1465-1501.

[Ab5] N. Abarenkova, J.-Ch. Anglès d'Auriac, S. Boukraa and J.-M. Maillard, Real topological entropy versus metric entropy for birational measurepreserving transformations, Physica D 144 (2000) 387-433.

[Ak] E. Akin, The General Topology of Dynamical Systems, AMS, 1993.

$[\mathrm{BD}]$ E. Bedford and J. Diller, Energy and invariant measure for bimeromorphic surface maps.

[BLS] E. Bedford, M. Lyubich, and J. Smillie, Polynomial diffeomorphisms of $\mathbf{C}^{2}$, IV. The measure of maximal entropy and laminar currents. Invent. Math., 112 (1993), 77-125.

[BS1] E. Bedford and J. Smillie, Real polynomial diffeomorphisms with maximal entropy: Tangencies. http://arXiv.org/math.DS/0103038

[BS2] E. Bedford and J. Smillie, Real polynomial diffeomorphisms with maximal entropy: II. Small Jacobian.

[BTR] M. Bernardo, T.T. Truong and G. Rollet, The discrete Painlevé I equations: transcendental integrability and asymptotic solutions, J. Phys. A: Math. Gen. 34 (2001), 3215-3252.

[BM] S. Boukraa and J.-M. Maillard, Factorization properties of birational mappings, Physica A 220 (1995), 403-470.

[dCH] A. de Carvalho and T. Hall, How to prune a horseshoe, Nonlinearity, 15 (2002), no. 3, R19-R68.

[DN] R. Devaney and Z. Nitecki, Shift automorphisms in the Hénon family, Comm. Math. Phys. 67 (1979), 137-146. 
[DF] J. Diller and C. Favre, Dynamics of bimeromorphic maps of surfaces, Amer. J. Math., 123 (2001), 1135-1169.

[Du] R. Dujardin, Dynamique d'applications non polynomiales et courants laminaires, Doctoral Thesis, Univ. de Paris Sud, Orsay, 2002.

[Fr] S. Friedland, Entropy of rational self-maps of projective varieties. In Dynamical Systems and Related Topics (Nagoya, 1990), pages 128-140. World Sci. Publishing, River Edge, NJ, 1991.

[FM] S. Friedland and J. Milnor, Dynamical properties of plane polynomial automorphisms, Ergod. Th. \& Dynam. Sys., 9 (1989), 67-99.

$[\mathrm{Fu}] \mathrm{W}$. Fulton, Intersection Theory, Ergebnisse der Mathematik und ihrer Grenzgebiete, Springer-Verlag, 1984.

[GNR] B. Grammaticos, F. Nijhoff and A. Ramani, Discrete Painlevé equations. The Painlevé property. (CRM Series in Mathematics Physics), (New York: Springer) (1999), 413-516.

[Gu1] V. Guedj, Dynamics of polynomial mappings of $\mathbf{C}^{2}$, Am. J. Math., 124 (2002), 75-106.

[Gu2] V. Guedj, Rational mappings with large topological degree, preprint.

[HO] J.H. Hubbard and R. Oberste-Vorth, Hénon mappings in the complex domain II: Projective and inductive limits of polynomials, Real and Complex Dynamical Systems (B. Branner and P. Hjorth, eds.), Kluwer, Boston, 1995, pp. 89-132.

[KH] A. Katok and B. Hasselblatt, Introduction to the Modern Theory of Dynamical Systems, Cambridge U. Press (1995).

[LM] D. Lind and B. Marcus, Symbolic Dynamics and Coding, Cambridge U. Press (1995).

[M] F. Morgan, Geometric Measure Theory, Academic Press, 1988.

[RS] D. Ruelle and D. Sullivan, Currents, flows and diffeomorphisms, Topology, 14 (1975), 319-327.

[S] N. Sibony, Dynamique des applications rationnelles de $\mathbf{P}^{k}$, Dynamique et géométrie complexes (Lyon, 1997), 97-185, Panor. Synthèses, 8, Soc. Math. France, Paris, 1999.

\footnotetext{
Department of Mathematics, Indiana University, Bloomington, IN 47405

E-mail address: bedford@indiana.edu

Department of Mathematics, University of Notre Dame, Notre Dame, IN 46556

E-mail address: diller.1@nd.edu
} 